\title{
Bulk-Boundary Correspondence for Disordered Free-Fermion Topological Phases
}

\author{
Alexander Alldridge ${ }^{1}$, Christopher Max $^{1,2}$ (D), Martin R. Zirnbauer ${ }^{2}$ \\ 1 Mathematisches Institut, Universität zu Köln, Cologne, Germany. E-mail: alldridg@math.uni-koeln.de \\ 2 Institut für Theoretische Physik, Universität zu Köln, Cologne, Germany. E-mail: cmax@ @hp.uni-koeln.de; \\ zirn@thp.uni-koeln.de
}

Received: 27 March 2019 / Accepted: 5 August 2019

Published online: 12 October 2019 - (C) The Author(s) 2019

\begin{abstract}
Guided by the many-particle quantum theory of interacting systems, we develop a uniform classification scheme for topological phases of disordered gapped free fermions, encompassing all symmetry classes of the Tenfold Way. We apply this scheme to give a mathematically rigorous proof of bulk-boundary correspondence. To that end, we construct real $\mathrm{C}^{*}$-algebras harbouring the bulk and boundary data of disordered free-fermion ground states. These we connect by a natural bulk-to-boundary short exact sequence, realising the bulk system as a quotient of the half-space theory modulo boundary contributions. To every ground state, we attach two classes in different pictures of real operator $K$-theory (or $K R$-theory): a bulk class, using Van Daele's picture, along with a boundary class, using Kasparov's Fredholm picture. We then show that the connecting map for the bulk-to-boundary sequence maps these $K R$-theory classes to each other. This implies bulk-boundary correspondence, in the presence of disorder, for both the "strong" and the "weak" invariants.
\end{abstract}

\section{Introduction}

The research field of topological quantum matter stands out by its considerable scope, pairing strong impact on experimental physics with mathematical structure and depth. On the experimental side, physicists are searching for novel materials with technological potential, e.g., for future quantum computers; on the theoretical side, mathematicians have been inspired to revisit and advance areas such as topological field theory and various types of generalised cohomology and homotopy theory.

Early research on topological quantum matter was concerned primarily with the integer and fractional quantum Hall effects (QHE) and close variants thereof. Many of the key principles were already recognised and developed in that context; on the mathematical side, these included the interpretation of the Hall conductance as a topological $K$-theory class $[5,51]$ and also as a Fredholm index [7,8], and the principle of bulk-boundary correspondence [50]. 
A major boost came around 2005 [26], when the research field was brought to full scale by the realisation that phenomena similar to QHE were possible even for systems with symmetries, in particular the anti-unitary symmetry of time reversal. This led to the notion of symmetry-protected topological phases as equivalence classes of gapped Hamiltonians that are connected by homotopies subject to a fixed symmetry group; see [17] for a recent review.

Rapid progress was made in the free-fermion limit with translation symmetry, where one can use the Fourier-Bloch technique to develop the theory over the Brillouin torus of conserved momentum. The challenge nowadays is to advance the theory in the direction of disordered and interacting systems. In the present paper, we focus on the disorder aspect, assuming that particle interactions can be handled by mean-field theory in its most general form, namely the so-called Hartree-Fock-Bogoliubov (HFB) mean-field approximation, or free-fermion approximation for short.

The traditional physics approach to disordered free fermions starts from the WegnerEfetov supersymmetry formalism, which famously led to the non-linear $\sigma$-model describing disordered metals. For systems with a small (if not vanishing) density of states, the non-linear $\sigma$-model has also been used, but it remains less well established there. In the present paper, we build on the mathematically rigorous approach developed initially for aperiodic systems and the integer quantum Hall effect by Bellissard and co-workers [8]. In that approach one assumes that translation invariance holds on average over the disorder and in that sense represents a symmetry of the macroscopic system.

A foundational aspect that has to be emphasised is the significant role played by superconductors, for theory as well as experiment. In fact, much of the excitement about topological quantum matter stems from the technological promise of robust zeroenergy modes (so-called Majorana zero modes) bound to the ends of superconducting wires of symmetry type $D$. To mention a second example of well documented interest, planar substructures in strontium ruthenate $\left(\mathrm{Sr}_{2} \mathrm{Ru} \mathrm{O}_{4}\right)$ behave as a two-dimensional topological superconductor with broken time-reversal symmetry [38].

Superconductivity is a true many-body phenomenon that requires the spontaneous breaking of the fundamental U(1) symmetry underlying charge conservation. As manybody systems with interactions and collective dynamics, superconductors defy any treatment based on straight single-particle quantum mechanics. Nonetheless, much of the phenomenology of superconductors and their quasi-particle excitations can be captured by the HFB mean-field approximation. In that scheme, one doubles the single-particle Hilbert space $\mathscr{V}$ to the Nambu space of fields, $\mathscr{W}=\mathscr{V} \oplus \mathscr{V}^{*}$, and then works with the adjoint action of the free-fermion Hamiltonians on the fields. The field space $\mathscr{W}$ is equipped with two canonical invariant structures: (i) a symmetric bilinear form due to the canonical anti-commutation relations for fermion fields; (ii) a real structure $\mathscr{W}_{\mathbb{R}} \subseteq \mathscr{W}$ known in physics as the space of Majorana fields. The resulting formalism, also known by the name of Bogoliubov-deGennes or Gorkov equations, is sometimes advertised as single-particle quantum mechanics with a "particle-hole symmetry". In the present work we do better: with a view towards future extensions to incorporate electron-electron interactions, we sketch how the formalism emerges from its proper foundations in many-body quantum theory, and we develop the theory accordingly.

Our main theme here will be bulk-boundary correspondence. Roughly speaking, this principle says that the topological characteristics of the bulk system must be reflected in corresponding properties of the boundary theory. In the general case of interacting systems, one has a trichotomy of possibilities: the boundary theory (i) may be gapless, 
or (ii) may undergo symmetry breaking, or (iii) may be a gapped topological phase of an exotic nature. For free fermions, however, only the first alternative is realised.

Historically, early indications of the principle of bulk-boundary correspondence came from anomalies in quantum field theory, especially the Adler-Bell-Jackiw anomaly for chiral fermions. Simply put, the boundary theory in isolation may be anomalous in that a conservation law is illegally violated and, if so, there must be an exactly opposite effect from the bulk system to cancel the total anomaly for the boundary joined with the bulk. For example, the low-energy effective action functional for Quantum Hall bulk systems contains a Chern-Simons term, which fails to be gauge-invariant when a boundary is present, and that gauge anomaly is cancelled by chiral boundary fermions that anomalously appear or disappear when magnetic flux crosses the boundary.

For the case of the integer quantum Hall effect, the heuristic principle of bulkboundary correspondence was established in mathematically rigorous terms by Kellendonk, Richter, and Schulz-Baldes [31,50]. The starting point of their approach is a short exact sequence of $\mathrm{C}^{*}$-algebras, realising the bulk system as a quotient of the half-space theory taken modulo the boundary theory. The short exact sequence induces a six-term exact sequence connecting the $K$-theory groups $K_{0}$ and $K_{1}$ of these $\mathrm{C}^{*}$ algebras. Recently, Prodan and Schulz-Baldes [43] have adapted and applied that early approach to the "chiral" analogue of IQHE systems in odd space dimension. As a result, there now exists a fairly complete understanding of the so-called "complex symmetry classes", known as type $A$ and $A$ III.

Several attempts have been made to give a mathematical proof of bulk-boundary correspondence for all Tenfold-Way symmetry classes. A notable result for $2 D$ systems in symmetry class $A$ II was proved by Graf-Porta [21]. The first approach encompassing all symmetry classes in $2 D$ is due to Loring [37], and is based on the formalism of almost commuting Hermitian matrices. Results covering all symmetry classes in any dimension were obtained by Hannabuss, Mathai, and Thiang. In a series of papers [23,39,40], they apply the concept of $T$-duality to the bulk-boundary correspondence. This duality, with its origins in string theory, relates the $K$-theory of two torus bundles over the same base manifold. In the presence of lattice symmetries, Kubota [34] establishes bulk-boundary correspondence at the level of strong invariants, by using the tools of coarse geometry. Following ideas of Freed-Moore [19], he combines the "quantum symmetries" of the Tenfold Way and the lattice symmetries into a single group. An alternative route, based on Kasparov $K K$-theory, is pursued by Bourne-Kellendonk-Rennie [13]. Building on the previous work of Bourne-Carey-Rennie [12], they show that certain bulk and boundary classes are related via the bulk-to-boundary map of Kellendonk-Richter-Schulz-Baldes.

Our approach to the bulk and boundary classification of free-fermion topological phases stands out by its foundation in the physically well-motivated Nambu space formalism. As explained above, this is essential for a proper treatment of topological superconductors. Since the objects we consider have a clear physical meaning, we are moreover able to draw conclusions with a bearing on the expected physics phenomenology; for example, our theory immediately yields the emergence of gapless edge modes for topologically non-trivial bulk phases.

Building on the pioneering work of Bellissard et al. [6-9], Schulz-Baldes et al. [31, $43,50]$, we give a complete and uniform proof of bulk-boundary correspondence for symmetry-protected topological (SPT) phases of free fermions with disorder. Our basic physics setting is that of the Tenfold Way of disordered free fermions [1], encompassing the two complex symmetry classes $A$ and $A$ III, as well as the eight "real symmetry classes" $D, D \mathrm{III}, A \mathrm{II}, C \mathrm{II}, C, C \mathrm{I}, A \mathrm{I}$, and $B D \mathrm{I}$. To avoid lengthy case-by-case considerations, 
we introduce the $\mathrm{C}^{*}$-algebras of Bellissard and Kellendonk-Richter-Schulz-Baldes in a form suitable for the introduction of "real" and "complex" symmetries, and we treat the symmetries systematically using Clifford algebras.

In Sect. 2, we begin by deriving the $\mathrm{C}^{*}$-algebra, $\mathbb{A}$, for the bulk in the Nambu space setting that emerges from the many-body theory. The construction puts the real structure of the Nambu space $\mathscr{W}$ into evidence, as is essential for our treatment of the symmetries appearing in the Tenfold Way. From our physics-driven model building, ground states of free-fermion Hamiltonians appear naturally as real skew-Hermitian unitaries $J$ on $\mathscr{W}$. These are called quasi-particle vacua (QPV) and figure as the principal objects of our further investigations. Ultimately, we work in the tight-binding approximation; we justify its use from physical principles. Adopting an idea due to Kubota [34], our construction of the bulk algebra $\mathbb{A}$ appeals to the uniform Roe $C^{*}$-algebra of the lattice $\Lambda$ of translations that leave the crystalline set of atomic sites invariant. That choice of $\mathrm{C}^{*}$-algebra reflects the (local) finiteness of the number of tight-binding degrees of freedom.

We incorporate disorder at this stage by following Bellissard [9] in encoding translational invariance on average through a covariance relation for the lattice $\Lambda$. It turns out (Theorem 1) that this covariance relation yields a particular structure for the $\mathrm{C}^{*}$-algebra $\mathbb{A}$, namely that of a crossed-product $\mathrm{C}^{*}$-algebra. Since the work of Bellissard, the bulk algebra has conventionally been defined as a crossed product; for us, this structure emerges naturally. To sum up, our real skew-Hermitian unitaries $J \in \mathbb{A}$ are translation-invariant on average over the disorder. We therefore call them disordered IQPV (where the I stands for invariant).

The next step is to include symmetries. Past treatments of the Tenfold Way have relied on case-by-case considerations, exacerbated further by the appearance of various case-dependent real and quaternionic structures. A uniform description avoiding these ramifications was first put forth by Kitaev [33] by the judicious use of Clifford algebras. It was later worked out in detail by Kennedy-Zirnbauer [32] who introduced the concept of pseudo-symmetries as an organising principle for symmetry classes of translationinvariant free fermions in the clean limit. In Sect. 3, we transcribe this concept from the Bloch-bundle setting to that of $\mathrm{C}^{*}$-algebras and use it to define symmetry classes of disordered IQPV $J \in \mathbb{A}$.

Our formulation turns out to be well suited for the introduction of $K$-theoretical invariants, and we show how a disordered IQPV $J \in \mathbb{A}$ naturally defines a class in the real $K$-theory of the bulk algebra $\mathbb{A}$ (Definition 16). The entirely algebraic construction utilises the picture of real $K$-theory introduced by Van Daele [52,53]. The use of this picture was inspired by recent work of Kellendonk [30]. Our construction indeed gives an alternative definition of real $K$-theory. That is, any $K R$-theory class of $\mathbb{A}$ is the "bulk class" of a disordered IQPV in a certain symmetry class (Theorem 3 ). This fact does not depend on the specifics of the bulk algebra $\mathbb{A}$ but is valid for any real $\mathrm{C}^{*}$-algebra.

In Sect. 4, we introduce a physical system boundary by defining a half-space algebra $\widehat{\mathbb{A}}$, following the ideas of Kellendonk-Richter-Schulz-Baldes [31,50]. We break the translational covariance in one spatial direction and show that the resulting algebra $\widehat{\mathbb{A}}$ has the structure of a crossed product with the half-space semilattice $\hat{\Lambda} \subseteq \Lambda$ (Proposition 8). This leads to a Toeplitz extension connecting bulk and boundary (Theorem 4 ).

The Szegó projection defining the Toeplitz extension allows us to assign, to every disordered IQPV $J$ in a given symmetry class, a real skew-Hermitian operator $\widehat{J} \in \widehat{\mathbb{A}}$ which satisfies the same pseudo-symmetries as $J$ but squares to -1 only up to boundary contributions. Such operators define real $K$-theory classes for the boundary $\mathrm{C}^{*}$-algebra 
$\mathbb{A}_{\partial}$, as we show by using Kasparov's Fredholm picture for $K R$-theory (Definition 21). Since for free fermions, the appearance of gapless edge modes signals a topologically non-trivial bulk, it is essential that the picture of $K$-theory used for the boundary classes allows the spectral gap to close at the boundary. This is an important aspect not present in previous approaches to the bulk-boundary correspondence using $K$-theory.

Thus, we attach to any disordered IQPV of a given symmetry class two $K$-theory classes: one in the bulk and another one at the boundary. The main result of our article, in Sect. 5, is to inter-relate these two classes: We prove in Theorem 5 that the relation is given by the connecting map in $K R$-theory. For the case of the integer QHE, this idea is already present in the work of Kellendonk-Richter-Schulz-Baldes [31,50]. Our theorem covers all symmetry classes of the Tenfold Way, and it holds in the presence of disorder, for the strong as well as the weak invariants. We also show, in Proposition 12, that the $K$-theory of the algebras we have constructed is invariant w.r.t. certain types of disorder.

In the final Sect. 6, we complement our results by a discussion, (i), of the anomalous nature of the boundary phase, (ii), of the role of the "trivial" phase, (iii), of the internal space of our tight-binding model, and (iv), of the construction of numerical topological invariants. We examine the so-called "strong" invariant and show the emergence, for topologically non-trivial systems, of gapless boundary phases.

Note on authorship. This article is part of the $\mathrm{PhD}$ thesis work of CM supervised by AA. The contributions of MZ are primarily in Sects. 1-3, where the mathematical model is developed from physical principles.

\section{Bulk Systems with Disorder}

2.1. From Nambu space to real $C^{*}$-algebras. In this subsection, we shall construct the algebra of bulk observables for a macroscopically translation invariant solid-state system in the free-fermion approximation and with on-site symmetries present.

In the literature on the subject, the $C^{*}$-algebra of a gapped bulk system is modelled by a crossed product $C^{*}$-algebra. This goes back to Bellissard [6,7], and it is fundamental for his mathematical proof of the (integer) quantisation of the Hall conductance using noncommutative geometry [8]. Later work by Schulz-Baldes-Kellendonk-Richter [50] and Kellendonk-Richter-Schulz-Baldes [31] employed a Toeplitz extension of the crossed product $C^{*}$-algebra of the bulk system to prove bulk-boundary correspondence for integer quantum Hall systems in a half-plane geometry. More recently, Prodan-Schulz-Baldes extended the formalism to incorporate half-space systems in the chiral symmetry class AIII (see [43, Chapter 5] for an exposition).

Our first goal here is to adapt the mathematical framework in order to accommodate free-fermion topological phases from the other symmetry classes of the Tenfold Way. For that purpose, we deem it fit to rebuild the $C^{*}$-algebra of our model from its foundations in many-body physics. This we shall do step-by-step in the present subsection, with the final outcome being that our algebra still has a crossed product structure. The latter will be of crucial importance in Sect. 4.

2.1.1. Setting in many-body theory Topological insulators and superconductors are many-body systems consisting of $10^{10}-10^{25}$ interacting particles. To treat them in any satisfactory way (including particle-particle interactions as well as disorder), one must apply the formalism of many-body quantum theory. In the present paper, we treat disordered systems, neglecting residual interactions beyond those captured by mean-field 
theory in the most general scheme of Hartree-Fock-Bogoliubov. While our focus on disorder makes for substantial technical simplifications, allowing us to do computations in the Nambu space of field operators (akin to the Hilbert space of single-particle quantum mechanics), we still consider it appropriate if not necessary to develop (or, at least, motivate) the mathematical model we use from its very foundations in many-body theory. A particular case in point is the proper description of topological superconductors. Indeed, superconductors are many-electron systems that undergo spontaneous breaking of U(1) charge symmetry, a many-body phenomenon that cannot be understood or modelled in a straight single-particle picture.

A basic characteristic of solid bodies and other condensed matter systems is their chemical potential $\mu$, also known as the Fermi energy in the case of particles obeying Fermi statistics. By definition, $\mu$ is the energy level at which the process of adding or removing a charged particle to or from a macroscopic body costs no work. Singleelectron states with energy above $\mu$ are called conduction states, those with energy below $\mu$ valence states. The single-electron conduction (resp. valence) states span a Hilbert space $\mathscr{V}_{+}\left(\right.$resp. $\left.\mathscr{V}_{-}\right)$.

Let us emphasise that it is crucial for us to construct our mathematical model around the chemical potential $\mu$. The reason is that we intend to truncate the locally infinite continuum degrees of freedom to a so-called tight-binding model supporting only a finite number of energy bands, and that approximation by truncation is physically justified (at very low temperatures) if and only if the tight-binding model is designed to capture the band structure close to the chemical potential.

The basic structure underlying many-body quantum theory is that of a Fock space. In the case of indistinguishable fermions such as electrons, this is an exterior algebra

$$
\bigwedge=\bigwedge\left(\mathscr{V}_{+} \oplus \mathscr{V}_{-}^{*}\right)
$$

completed to a Hilbert space with inner product $\langle\cdot \mid \cdot\rangle_{\wedge}$ which is induced by the Hilbert space structures of $\mathscr{V}_{ \pm}$. Note that $\mathscr{V}_{-}^{*}$ denotes the vector space dual to $\mathscr{V}_{-}$. Physically speaking, the appearance of $\mathscr{V}_{-}^{*}$ (instead of $\mathscr{V}_{-}$) means that the Fock vacuum, i.e. the complex line

$$
\bigwedge^{0}=\bigwedge^{0}\left(\mathscr{V}_{+} \oplus \mathscr{V}_{-}^{*}\right) \cong \mathbb{C}
$$

is the "Fermi sea" of occupied valence states. The Fock space $\bigwedge$ is bi-graded by

$$
\bigwedge=\bigoplus_{p, q \geqslant 0} \bigwedge^{p, q}, \quad \bigwedge^{p, q}:=\bigwedge^{p}\left(\mathscr{V}_{+}\right) \otimes \bigwedge^{q}\left(\mathscr{V}_{-}^{*}\right)
$$

The integer $p$ counts the number of single-particle excitations, i.e. the number of electrons populating the conduction states, while $q$ is the number of single-hole excitations, i.e. the number of electrons missing from the Fermi sea of occupied valence states. Abbreviating the language, one calls $p$ and $q$ the number of particles resp. of holes. The difference $p-q$ is referred to as the charge (relative to $\mu$ ).

Given the Fock-Hilbert space $\bigwedge$ built on the Fock vacuum $\bigwedge^{0}$ around the chemical potential $\mu$, a field operator $\psi: \bigwedge \longrightarrow \bigwedge$ is a linear combination

$$
\psi=\varepsilon\left(v_{+}\right)+\iota\left(\varphi_{+}\right)+\iota\left(v_{-}\right)+\varepsilon\left(\varphi_{-}\right) \quad\left(v_{ \pm} \in \mathscr{V}_{ \pm}, \varphi_{ \pm} \in \mathscr{V}_{ \pm}^{*}\right)
$$

Here, the operator of exterior multiplication $\varepsilon\left(v_{+}\right): \bigwedge^{p, q} \rightarrow \bigwedge_{p, q+1} \bigwedge^{p+1, q}$ increases the number of particles by one, whereas $\varepsilon\left(\varphi_{-}\right): \bigwedge^{p, q} \longrightarrow \bigwedge^{p, q+1}$ removes an electron from a valence state, thereby increasing the number of holes by one. Conversely, the 
operators $\iota\left(\varphi_{+}\right): \bigwedge^{p, q} \longrightarrow \bigwedge^{p-1, q}$ and $\iota\left(v_{-}\right): \bigwedge^{p, q} \longrightarrow \bigwedge^{p, q-1}$ decrease the number of particles resp. holes by alternating contraction.

The field operators $\psi$ span a complex vector space, the so-called Nambu space $\mathscr{W}$ of fields. Note that $\mathscr{W}$ is not fundamentally a Hilbert space, but will be given a derived Hilbert space structure in due course. The space $\mathscr{W}$ is equipped with two basic structures. The first one is a symmetric complex bilinear form, $\{\cdot, \cdot\}: \mathscr{W} \otimes \mathscr{W} \longrightarrow \mathbb{C}$, which is canonically defined by

$$
\psi \psi^{\prime}+\psi^{\prime} \psi=\left\{\psi, \psi^{\prime}\right\} \mathrm{id}_{\wedge} .
$$

It is referred to as the CAR form (for canonical anticommutation relations). In explicit terms, using the notation above,

$$
\left\{\psi, \psi^{\prime}\right\}=\varphi_{+}\left(v_{+}^{\prime}\right)+\varphi_{-}\left(v_{-}^{\prime}\right)+\varphi_{+}^{\prime}\left(v_{+}\right)+\varphi_{-}^{\prime}\left(v_{-}\right) .
$$

The most general many-body operator acting on the Fock space resides in the complex Clifford algebra $C \ell(\mathscr{W})$ generated by $\mathscr{W}$ with the relations given by the CAR form.

Secondly, the Nambu space of fields carries a real structure $\gamma: \mathscr{W} \longrightarrow \mathscr{W}$. Denoting the Fréchet-Riesz isomorphism for the conduction-state and valence-state Hilbert spaces by $h: \mathscr{V}_{ \pm} \longrightarrow \mathscr{V}_{ \pm}^{*}$, one defines $\gamma$ as the anti-linear involution

$$
\varepsilon\left(v_{+}\right)+\iota\left(\varphi_{+}\right)+\iota\left(v_{-}\right)+\varepsilon\left(\varphi_{-}\right) \longmapsto \iota\left(h v_{+}\right)+\varepsilon\left(h^{-1} \varphi_{+}\right)+\varepsilon\left(h v_{-}\right)+\iota\left(h^{-1} \varphi_{-}\right) .
$$

Physicists frequently refer to the real subspace $\mathscr{W}_{\mathbb{R}}$ consisting of the $\gamma$-fixed vectors as the space of Majorana fields. Notice that the CAR form of $\mathscr{W}$ restricts to a Euclidean structure of the real vector space $\mathscr{W}_{\mathbb{R}}$.

The real structure $\gamma$ and the CAR form of $\mathscr{W}$ each induce a corresponding operation on linear transformations $L \in \operatorname{End}(\mathscr{W})$ (and similarly on the anti-linear transformations). In the case of $\gamma$, this is the anti-linear involution

$$
L \longmapsto \bar{L}:=\gamma \circ L \circ \gamma
$$

and we say that $L$ is real if $\bar{L}=L$ (resp. imaginary if $\bar{L}=-L$ ). In the case of the CAR form, the induced operation is a linear anti-involution $L \longmapsto L^{\top}$ called transposition, defined by

$$
\left\{\psi, L^{\top} \psi^{\prime}\right\}=\left\{L \psi, \psi^{\prime}\right\} \quad\left(\psi, \psi^{\prime} \in \mathscr{W}\right) .
$$

An operator $L \in \operatorname{End}(\mathscr{W})$ is called skew if $L^{\top}=-L$ (resp. symmetric if $L^{\top}=L$ ).

Finally, let us remark here that the $\operatorname{CAR}$ form $\{\cdot, \cdot\}$ and the real structure $\gamma$ are fundamental in that they are not tied to the free-fermion approximation but retain their key roles even in the presence of electron-electron interactions.

2.1.2. Free-fermion setting We proceed to introduce the objects and structures that emerge upon assuming the free-fermion approximation (or free-fermion limit). For $\psi, \psi^{\prime} \in \mathscr{W}_{\mathbb{R}}$, consider the expression $\psi \psi^{\prime}-\psi^{\prime} \psi$ in the Clifford algebra $C \ell\left(\mathscr{W}_{\mathbb{R}}\right)$. Such operators, quadratic in the fields and skew-symmetrised, close under the commutator in $\operatorname{End}(\bigwedge)$ and span a Lie algebra isomorphic to $\mathfrak{s o}\left(\mathscr{W}_{\mathbb{R}}\right)$. By exponentiating that Lie algebra inside $C \ell\left(\mathscr{W}_{\mathbb{R}}\right)$, one obtains the Lie group $\operatorname{Spin}\left(\mathscr{W}_{\mathbb{R}}\right) \subseteq \mathrm{U}(\bigwedge)$. The latter acts on $\mathscr{W}_{\mathbb{R}} \subseteq \operatorname{End}(\bigwedge)$ by real orthogonal transformations $\varrho(g): \mathscr{W}_{\mathbb{R}} \longrightarrow \mathscr{W}_{\mathbb{R}}$, defined as

$$
\varrho(g) \psi:=g \psi g^{-1} \quad\left(g \in \operatorname{Spin}\left(\mathscr{W}_{\mathbb{R}}\right)\right)
$$


The significance of $\operatorname{Spin}\left(\mathscr{W}_{\mathbb{R}}\right)$ is that it contains all unitary operators of physical interest (in the free-fermion limit), including the operators for time evolutions, space translations, space rotations, etc., as well as the unitary Bogoliubov transformations.

A free-fermion Hamiltonian $\mathscr{H} \in C \ell(\mathscr{W})$ is, by definition, a Clifford algebra element quadratic in the fields and Hermitian with respect to the Fock space inner product. Because the time evolutions $e^{-i t \mathscr{H} / \hbar} \in \operatorname{Spin}\left(\mathscr{W}_{\mathbb{R}}\right)$ generated by it act on $\mathscr{W}_{\mathbb{R}}$ as real orthogonal transformations $\varrho\left(e^{-i t \mathscr{H} / \hbar}\right) \in \mathrm{SO}\left(\mathscr{W}_{\mathbb{R}}\right)$, it follows that $i H=d \varrho(i \mathscr{H})$ is real and skew: $\overline{i H}=+i H=-i H^{\top}$. Put differently, the induced Hamiltonian $H \in$ $\operatorname{End}(\mathscr{W})$ is imaginary and skew:

$$
\bar{H}=-H=H^{\top} .
$$

Let us provide a little more detail about the free-fermion Hamiltonians $\mathscr{H}$. If $\mathscr{H}$ stems from a charge-conserving Hamiltonian $\mathrm{H}$ which is defined on the single-particle Hilbert space $\mathscr{V}=\mathscr{V}_{+} \oplus \mathscr{V}_{-}$and decomposed as

$$
\mathrm{H}=\left(\begin{array}{ll}
A & B \\
C & D
\end{array}\right) \in\left(\begin{array}{cc}
\operatorname{End}\left(\mathscr{V}_{+}\right) & \operatorname{Hom}\left(\mathscr{V}_{-}, \mathscr{V}_{+}\right) \\
\operatorname{Hom}\left(\mathscr{V}_{+}, \mathscr{V}_{-}\right) & \operatorname{End}\left(\mathscr{V}_{-}\right)
\end{array}\right),
$$

then for any bases $\left(e_{j}^{\tau}\right)$ of $\mathscr{V}_{\tau}(\tau= \pm)$ with dual bases $\left(f_{\tau}^{j}\right)$, one has the second-quantised Hamiltonian

$$
\begin{aligned}
\mathscr{H}:= & \sum_{j} \varepsilon\left((A-\mu) e_{j}^{+}\right) \iota\left(f_{+}^{j}\right)+\sum_{\ell} \varepsilon\left(B e_{\ell}^{-}\right) \varepsilon\left(f_{-}^{\ell}\right) \\
& -\sum_{j} \iota\left(f_{+}^{j}\right) \iota\left(C e_{j}^{+}\right)-\sum_{\ell} \varepsilon\left(f_{-}^{\ell}\right) \iota\left((D-\mu) e_{\ell}^{-}\right) .
\end{aligned}
$$

Thus, the block $B \in \operatorname{Hom}\left(\mathscr{V}_{-}, \mathscr{V}_{+}\right)$gives rise to an operator of type $\varepsilon \varepsilon$ creating particlehole pairs, while the block $C$ (second-quantised as type $\iota \iota$ ) does the opposite. If $\mathrm{H}$ is taken to be exactly the Hartree-Fock mean-field Hamiltonian, then $B=C=0$ and $A-\mu>0>D-\mu$ by the definition of $\mathscr{V}_{+}$and $\mathscr{V}_{-}$from the chemical potential $\mu$. The charge operator (representing the identity on $\mathscr{V}$ ) is

$$
\mathscr{Q}=\sum_{j} \varepsilon\left(e_{j}^{+}\right) \iota\left(f_{+}^{j}\right)-\sum_{\ell} \varepsilon\left(f_{-}^{\ell}\right) \iota\left(e_{\ell}^{-}\right) .
$$

Note that $\left.\mathscr{Q}\right|_{\wedge p, q}=p-q$.

The full power of the formalism comes to play in the treatment of superconducting systems. There, the $U(1)$ symmetry underlying charge conservation is spontaneously broken (because of many-body effects such as the interaction of the electrons with lattice vibrations favouring the formation of so-called Cooper pairs). In the Hartree-FockBogoliubov mean-field approximation, one accounts for the spontaneous breaking of $\mathrm{U}(1)$ symmetry by augmenting the free-fermion Hamiltonian $\mathscr{H}$ with so-called pairing terms $\Delta_{+}$and $\Delta_{-}$that do not conserve the charge. Rather, they satisfy

$$
\left[\mathscr{Q}, \Delta_{ \pm}\right]= \pm 2 \Delta_{ \pm}
$$

and thus increase or decrease the charge by 2 units.

To summarise and simplify the notation, let us record here that any free-fermion Hamiltonian $\mathscr{H}$ (superconducting or not) acts on the Nambu space $\mathscr{W}$ of fields $\psi$ as

$$
H \psi=i^{-1} d \rho(i \mathscr{H}) \psi=[\mathscr{H}, \psi] .
$$


Now, the ground state of a gapped free-fermion Hamiltonian $\mathscr{H}$ or, equivalently, of the induced Hamiltonian $H$, is known to be uniquely determined by the flat-band (or flattened) Hamiltonian

$$
J:=-i \operatorname{sign}(H)
$$

where a factor $i=\sqrt{-1}$ was inserted to make $J$ real $(\bar{J}=J)$. Note that $\operatorname{sign}(H)=$ $H|H|^{-1}$ is well-defined by the hypothesis of a non-vanishing energy gap. We then infer the relation $J^{2}=-1$, which makes $J$ a complex structure of the real vector space $\mathscr{W}_{\mathbb{R}}$. Recalling that $H$ is skew under transposition, we see that the same property holds for $J$ (i.e. $J^{\top}=-J$ ). It then follows from $J^{\top} J=-J^{2}=1$ that $J$ preserves the CAR form:

$$
\left\{J \psi, J \psi^{\prime}\right\}=\left\{\psi, \psi^{\prime}\right\}
$$

and is thus an orthogonal transformation. In summary, $J=-i \operatorname{sign}(H)$ is a CARpreserving complex structure of the space of Majorana fields $\mathscr{W}_{\mathbb{R}}$.

Let us briefly explain why the full information about the free-fermion ground state is encoded precisely in the complex structure $J$. The operator $J$ has two eigenspaces, $\operatorname{ker}(J \mp i) \subseteq \mathscr{W}_{\mathbb{R}} \otimes_{\mathbb{R}} \mathbb{C}=\mathscr{W}$. These eigenspaces provide a polarisation:

$$
\mathscr{W}=\mathscr{A}^{\mathrm{c}} \oplus \mathscr{A}, \quad \mathscr{A}^{\mathrm{c}}=\operatorname{ker}(J+i), \quad \mathscr{A}=\operatorname{ker}(J-i),
$$

and they determine $J$. Because $J$ preserves the CAR form, the subspaces $\mathscr{A}$ and $\mathscr{A}^{\mathrm{c}}$ are isotropic:

$$
\{\mathscr{A}, \mathscr{A}\}=0=\left\{\mathscr{A}^{\mathrm{c}}, \mathscr{A}^{\mathrm{c}}\right\} \text {. }
$$

For $a \in \mathscr{A}, c \in \mathscr{A}^{\mathrm{c}}$, and $\mathscr{J}=d \rho^{-1}(J)$, one has

$$
[i \mathscr{J}, a]=i J a=-a, \quad[i \mathscr{J}, c]=i J c=+c .
$$

Thus the field operators in $\mathscr{A}$ lower the energy of the flattened Hamiltonian $i \mathscr{J}$, while those in $\mathscr{A}^{\mathrm{c}}$ increase it. Clearly, the ground state as the state of lowest energy must be annihilated by the field operators that lower the energy. It follows that the isotropic subspace $\mathscr{A}$ furnishes all the (quasi-particle) annihilation operators. These, by a basic result of many-body theory, precisely characterise the free-fermion ground state. We mention in passing that the polarisation provided by the bare vacuum,

$$
\mathscr{W}=\mathscr{V} \oplus \mathscr{V}^{*}, \quad \mathscr{V}=\mathscr{V}_{+} \oplus \mathscr{V}_{-},
$$

would be a poor starting point on which to build an effective theory describing the low-energy physics that takes place near the chemical potential.

Altogether, the following definition is now well-motivated.

Definition 1. A free-fermion ground state or quasi-particle vacuum (QPV) is a complex structure $J$ that preserves the CAR form:

$$
J=-J^{-1}=-J^{\top} \in \mathscr{L}\left(\mathscr{W}_{\mathbb{R}}\right) .
$$

Here, $\mathscr{L}(-)$ denotes the set of bounded linear operators.

Remark 1. For translation-invariant systems, $J$ is diagonal in the momentum $k$. In that case, the orthogonality condition $J^{\top}=J^{-1}$ refines to $J(-k)^{\top}=J(k)^{-1}$. The latter is equivalent to a condition on the spaces of quasi-particle annihilation operators: $\{\mathscr{A}(k), \mathscr{A}(-k)\}=0$ which is the Fermi constraint of Kennedy-Zirnbauer [32]. 
2.1.3. Hilbert space structure of $\mathscr{W}$ Looking at the bigger picture one realises that, up to the present point, a similar development could have been carried out for bosons instead of fermions. In fact, a free-boson ground state is precisely a CCR-preserving complex structure of the real space of fields $\mathscr{W}_{\mathbb{R}}$ viewed as a symplectic vector space (where CCR stands for canonical commutation relations). In the next step, however, we come to a structure specific to fermions in the free-fermion limit: $\mathscr{W}$ is a complex Hilbert space, as follows.

Recall that the CAR form $\{\cdot, \cdot\}$ on $\mathscr{W}$ is symmetric and complex bilinear, and restricts to a Euclidean structure of $\mathscr{W}_{\mathbb{R}}$. For $\psi \in \mathscr{W}$, let

$$
\psi=\psi_{R}+i \psi_{I}, \quad \psi_{R}:=\frac{1}{2}(\psi+\gamma \psi), \quad \psi_{I}:=\frac{1}{2 i}(\psi-\gamma \psi)
$$

be the decomposition into real and imaginary parts. Then

$$
\left\langle\psi \mid \psi^{\prime}\right\rangle_{\mathscr{W}}:=\left\{\psi_{R}-i \psi_{I}, \psi_{R}^{\prime}+i \psi_{I}^{\prime}\right\}
$$

is an inner product turning $\mathscr{W}$ into a Hermitian vector space. In other words:

Definition 2 (Inner product on Nambu space). The Nambu space $\mathscr{W}$ of fermion field operators is equipped with an inner product $\langle\cdot \mid \cdot\rangle \mathscr{W}$ by

$$
\left\langle\psi \mid \psi^{\prime}\right\rangle_{\mathscr{W}}=\left\{\gamma \psi, \psi^{\prime}\right\} \quad\left(\psi, \psi^{\prime} \in \mathscr{W}\right)
$$

Thus, $\mathscr{W}$ acquires the structure of a complex Hilbert space.

Remark 2. The Hilbert space structure on $\mathscr{W}$ depends on our free-fermion assumption in two respects:

(i) The same construction for bosons leads to an inner product with indefinite signature, and for this reason not to a Hilbert space.

(ii) The invariance of $\langle\psi \mid \psi\rangle_{\mathscr{W}} \geqslant 0$ under unitary time evolutions can be interpreted as a law of probability conservation for quasi-particles. That law is violated by electronelectron interactions, so $\langle\cdot \mid \cdot\rangle_{\mathscr{W}}$ ceases to be relevant for interacting systems.

The presence of the inner product entails an operation End( $\mathscr{W}) \ni L \longmapsto L^{*}$ of taking the Hermitian adjoint. By construction, this is the same as the operation of transpose combined with that of $\gamma$-conjugate:

$$
L^{*}=(\bar{L})^{\top}
$$

Now, recall from Definition 1 the notion of a free-fermion ground state or quasi-particle vacuum $(\mathrm{QPV})$. Because any QPV $J \in \mathscr{L}(\mathscr{W} \mathbb{R})$ is skew, its complex linear extension $J \in \mathscr{L}(\mathscr{W})$ is skew-Hermitian $\left(J^{*}=-J\right)$. It follows that $J$, being a complex structure $J=-J^{-1}$ of $\mathscr{W} \mathbb{R}$, extends to a unitary operator $J=\left(J^{-1}\right)^{*}$ on $\mathscr{W}$.

Proposition 1. Viewed as an operator on the complex Hilbert space $\mathscr{W}$, any quasiparticle vacuum $J$ is skew-Hermitian and unitary:

$$
J^{*}=-J=J^{-1},
$$

as well as real: $\bar{J}=J$. 
As we have stressed above, the Hilbert space structure, unlike the CAR form and the real structure, is not a fundamental feature of the Nambu space $\mathscr{W}$. However, as long as we limit our attention to the free-fermion case, it is permissible mathematically to upend the logic and put the Hilbert space structure first. This is convenient for the purely pragmatic purpose of connecting us to the mathematical literature. Accordingly, we pose the following definition.

Definition 3 (Real Hilbert spaces). Let $\mathscr{W}$ be a complex Hilbert space with inner product $\langle\cdot \mid \cdot\rangle$. A real structure on $\mathscr{W}$ is an anti-unitary involution $\gamma$. That is, $\gamma^{2}=1$ and

$$
\left\langle\gamma \psi \mid \gamma \psi^{\prime}\right\rangle=\left\langle\psi^{\prime} \mid \psi\right\rangle \quad\left(\psi, \psi^{\prime} \in \mathscr{W}\right) .
$$

A real Hilbert space is by definition a pair $(\mathscr{W}, \gamma)$ of a Hilbert space $\mathscr{W}$ and a real structure $\gamma$. A complex linear (or anti-linear) map $\phi:\left(\mathscr{W}_{1}, \gamma_{1}\right) \longrightarrow\left(\mathscr{W}_{2}, \gamma_{2}\right)$ is called real if it intertwines the real structures, i.e.

$$
\phi \circ \gamma_{1}=\gamma_{2} \circ \phi
$$

In the framework of this definition, the Nambu space $\mathscr{W}$, equipped with its natural real structure, is a real Hilbert space, and any QPV $J$ is a real skew-Hermitian unitary.

2.1.4. Tight-binding approximation A major benefit from developing the mathematical model from its proper foundations in many-body quantum mechanics, is that we can now introduce a tight-binding approximation in a mathematically justified and physically meaningful way. (This will be important for our treatment of weak topological invariants; $c f$. Sect. 6. In fact, without proper theory building, the weak invariants might be criticised as artefacts due to the illegal step of replacing the spatial continuum by a discrete approximation.)

Let us recall that for a many-fermion system at very low temperatures, the active degrees of freedom reside near the chemical potential $\mu$. More precisely, the low-energy excitations are single particles in the conduction bands just above $\mu$ and single holes in the valence bands just below $\mu$. Hence, to capture the topological properties of the ground state (and other low-temperature phenomena), one may prune the locally infinite number of freedoms in the full single-particle Hilbert space, by assuming a truncation to the low-energy sector spanned by the conduction and valence bands near $\mu$. In this vein, we now make the following choice for our Hilbert spaces $\mathscr{V}_{+}$and $\mathscr{V}_{-}$:

$$
\mathscr{V}_{\tau}=\ell^{2}(\Lambda) \otimes V_{\tau} \quad(\tau= \pm)
$$

with lattice $\Lambda \cong \mathbb{Z}^{d}$. The factor $\ell^{2}(\Lambda)$ is the Hilbert space of a so-called tight-binding model, where the lattice $\Lambda \cong \mathbb{Z}^{d}$ reflects the organisation of the crystal by atomic sites. (In fact, the atomic sites may form a more complex pattern or unit cell invariant under the translational action of the $\mathbb{Z}^{d}$ lattice; in this paper, we focus on systems homogeneous w.r.t. translations, ignoring any further spatial symmetries.) The finitedimensional factors $V_{ \pm}$account for the spin and orbital degrees of freedom active in the conduction bands $\left(V_{+}\right)$and the valence bands $\left(V_{-}\right)$near the chemical potential. The resulting mathematical model is summarised as follows.

The Nambu space of field operators is

$$
\mathscr{W}=\mathscr{V}_{+} \oplus \mathscr{V}_{-}^{*} \oplus\left(\mathscr{V}_{+} \oplus \mathscr{V}_{-}^{*}\right)^{*}=\mathscr{V} \oplus \mathscr{V}^{*}, \quad \mathscr{V}:=\mathscr{V}_{+} \oplus \mathscr{V}_{-}
$$


Here, we may identify

$$
\mathscr{V} \cong \ell^{2}(\Lambda) \otimes V, \quad V:=V_{+} \oplus V_{-} .
$$

By the procedure outlined above, $\mathscr{W}$ acquires the structure of a real Hilbert space. Using the inner product and the (complex) conjugation on $\ell^{2}(\Lambda)$, we may identify

$$
\mathscr{W} \cong \ell^{2}(\Lambda) \otimes W, \quad W:=V \oplus V^{*}
$$

The splitting $\mathscr{V} \oplus \mathscr{V}^{*}$ of $\mathscr{W}$ is characterised by the (de-quantised) charge operator

$$
Q:=\left(\begin{array}{cc}
1 & 0 \\
0 & -1
\end{array}\right): \mathscr{W}=\mathscr{V} \oplus \mathscr{V}^{*} \longrightarrow \mathscr{W},
$$

resulting via $Q=i^{-1} d \rho(i \mathscr{Q})$ from the second-quantised charge operator $\mathscr{Q}$ introduced earlier. Observe that an arbitrary operator on $\mathscr{W}$ leaves $\mathscr{V}$ and $\mathscr{V}^{*}$ invariant if and only if it commutes with $Q$. Since $i Q$ generates the U(1) symmetry group underlying the conservation of charge, we call such operators charge-conserving. Charge-conserving operators with the additional property of being real take the general form

$$
O=\left(\begin{array}{cc}
\left.O\right|_{\mathscr{V}} & 0 \\
0 & h\left(\left.O\right|_{\mathscr{V}}\right) h^{-1}
\end{array}\right)
$$

where $\left.h \equiv \gamma\right|_{\mathscr{V}}: \mathscr{V} \longrightarrow \mathscr{V}^{*}$ is the Fréchet-Riesz isomorphism of the Hilbert space $\mathscr{V}$. Thus, any such operator is determined by its restriction to $\mathscr{V}$.

2.1.5. Real $C^{*}$-algebras As derived above, a key feature of our mathematical model is the presence of a real structure. Using a convenient mathematical short cut, the definition of a free-fermion ground state or QPV $J$ can be formulated thus:

$$
\bar{J}=J, \quad J^{*}=-J, \quad J^{2}=-1 ;
$$

that is, by reference only to the operations of forming the $\gamma$-conjugate, the Hermitian adjoint, and the product of operators.

Our aim, in the remainder of the current subsection, is to formulate an algebraic framework for the study of free-fermion topological phases in the presence of disorder. This will enable us to bring the well-developed theory of operator $K$-theory to bear upon the problem of bulk and boundary classification of such phases.

Defined algebraically as a (real) skew-Hermitian unitary, the free-fermion QPV J resides in the algebra $\mathscr{L}(\mathscr{W})$ of bounded linear operators on $\mathscr{W}$. By a classical theorem of Gel'fand and Naimark (see [42, Corollary 3.7.5]), the closed subalgebras of the algebra $\mathscr{L}(\mathscr{H})$ of bounded operators on a (complex) Hilbert space $\mathscr{H}$ invariant under the formation of Hermitian adjoints can be defined algebraically without reference to the Hilbert space. The algebraic objects so defined are exactly the so-called $\mathrm{C}^{*}$-algebras.

In addition to the Hermitian adjoint, real $C^{*}$-algebras formalise the notion of a real structure; they are to real Hilbert spaces what $\mathrm{C}^{*}$-algebras are to Hilbert spaces. Abstractly, they are defined as follows. 
Definition 4 (Real $C^{*}$-algebras). A real $C^{*}$-algebra is a pair $(A, \overline{-})$ consisting of a (complex) $C^{*}$-algebra $A$ and an anti-linear $*$-involution ${ }^{*}$ called conjugation; viz.

$$
\overline{x y}=\bar{x} \bar{y}, \quad \overline{x^{*}}=(\bar{x})^{*}, \quad \overline{\lambda x+y}=\bar{\lambda} \bar{x}+\bar{y} \quad(x, y \in A, \lambda \in \mathbb{C}) .
$$

An element $x \in A$ is called real if $\bar{x}=x$ and imaginary if $\bar{x}=-x$. A $*$-morphism $\phi:\left(A_{1},{ }^{-}\right) \longrightarrow\left(A_{2},{ }^{-}\right)$of real $C^{*}$-algebras is called real if it intertwines the conjugations, i.e.

$$
\phi(\bar{x})=\overline{\phi(x)} \quad\left(x \in A_{1}\right) .
$$

The spatial tensor product $A_{1} \otimes A_{2}$ of $C^{*}$-algebras, where $\left(A_{1}, \cdot\right)$ and $\left(A_{2},{ }^{\circ}\right)$ are real $\mathrm{C}^{*}$-algebras, becomes a real $\mathrm{C}^{*}$-algebra when equipped with the conjugation defined by

$$
\overline{x_{1} \otimes x_{2}}:=\overline{x_{1}} \otimes \overline{x_{2}} \quad\left(x_{1} \in A_{1}, x_{2} \in A_{2}\right)
$$

Remark 3. Equivalently, a real $\mathrm{C}^{*}$-algebra is characterised as a $\mathrm{C}^{*}$-algebra $A$, together with a linear anti-involution $\mathrm{T}$ called transposition. That is, $\mathrm{T}$ is complex linear and

$$
(x y)^{\top}=y^{\top} x^{\top}, \quad\left(x^{*}\right)^{\top}=\left(x^{\top}\right)^{*}, \quad\left(x^{\top}\right)^{\top}=x \quad(x, y \in A) .
$$

The two equivalent structures are related by the equation

$$
x^{\top}=\bar{x}^{*} \quad(x \in A) .
$$

In our setting based on the Nambu space of fields, $\mathscr{W}$, the basic example of a real $\mathrm{C}^{*}$-algebra is a closed $*$-subalgebra $A \subseteq \mathscr{L}(\mathscr{W})$ that is invariant under conjugation (or equivalently, under transposition with respect to the CAR form). Within any such algebra, we will be able to define the notion of a QPV.

From an abstract point of view, all real $\mathrm{C}^{*}$-algebras are realised on a real Hilbert space, as the following remark implies.

Remark 4 . For any real $\mathrm{C}^{*}$-algebra $\left.(A,)^{-}\right)$, the real subalgebra $B=A_{\mathbb{R}}$ consisting of all real elements is a real Banach $*$-algebra such that for every $x \in B,\left\|x^{*} x\right\|=\|x\|^{2}$ and $1+x^{*} x$ is invertible in the unitisation of $B$. Building on work of Arens [2] and Arens-Kaplansky [3] in the commutative case, Ingelstam [25] proved that conversely, if $B$ is a real Banach $*$-algebra satisfying these hypotheses, then its complexification $A=B_{\mathbb{C}}$ is a real $C^{*}$-algebra for the conjugation given by

$$
\overline{x+i y}:=x-i y \quad(x, y \in B) .
$$

Moreover, he proved that every real $\mathrm{C}^{*}$-algebra $\left(A,{ }^{-}\right)$admits, for some real Hilbert space $(\mathscr{W}, \gamma)$, a real isometric $*$-isomorphism onto a closed subalgebra of $\mathscr{L}(\mathscr{W})$ which is invariant under the adjoint and the transpose.

Although real $\mathrm{C}^{*}$-algebras can thus always be realised on a real Hilbert space, in practice, they also arise through quaternionic rather than through real structures. This will be important for our treatment of time reversal.

Definition 5 (Quaternionic structures on Hilbert space). Let $\mathscr{V}$ be a Hilbert space (over the complex numbers). An anti-unitary operator $T$ on $\mathscr{V}$ is called a quaternionic structure if $T^{2}=-1$. That is, $T$ is anti-linear and we have

$$
\left\langle T v_{1} \mid T v_{2}\right\rangle=\left\langle v_{2} \mid v_{1}\right\rangle, \quad\left\langle T v_{1} \mid v_{2}\right\rangle=-\left\langle T v_{2} \mid v_{1}\right\rangle \quad\left(v_{1}, v_{2} \in \mathscr{V}\right)
$$


A pair $(\mathscr{V}, T)$ consisting of a Hilbert space $\mathscr{V}$ and a quaternionic structure $T$ is called a quaternionic Hilbert space. A complex linear map $\phi:\left(\mathscr{W}_{1}, T_{1}\right) \longrightarrow\left(\mathscr{W}_{2}, T_{2}\right)$ between quaternionic Hilbert spaces is called quaternionic if it intertwines the quaternionic structures, viz.

$$
\phi \circ T_{1}=T_{2} \circ \phi .
$$

Let $(\mathscr{V}, T)$ be a quaternionic Hilbert space. Then $\mathscr{L}(\mathscr{V})$ is a real $\mathrm{C}^{*}$-algebra with the conjugation

$$
\bar{A}:=T^{*} A T=-T A T \quad(A \in \mathscr{L}(\mathscr{V})) .
$$

In the context of our applications, a quaternionic structure on $\mathscr{V}=\ell^{2}(\Lambda) \otimes V$ arises as $\mathrm{c} \otimes T$, where $\mathrm{c}$ is complex conjugation on $\ell^{2}(\Lambda)$ and $T$ is a quaternionic structure on $V$, corresponding to the operation of time reversal.

An example of a quaternionic structure is given for $V=\mathbb{C}^{2}$ by

$$
\mathfrak{c}:=\left(\begin{array}{cc}
0 & c \\
-\mathrm{c} & 0
\end{array}\right)
$$

where $\mathrm{c}$ is complex conjugation on $\mathbb{C}$. This induces on $M_{2}(\mathbb{C})=\operatorname{End}\left(\mathbb{C}^{2}\right)$ the conjugation $^{-}=\operatorname{Ad}(\mathfrak{c})$, expressed explicitly by the formula

$$
\overline{\left(\begin{array}{ll}
a & b \\
c & d
\end{array}\right)}=\left(\begin{array}{cc}
\bar{d} & -\bar{c} \\
-\bar{b} & \bar{a}
\end{array}\right) \quad(a, b, c, d \in \mathbb{C}) .
$$

For the real $C^{*}$-algebra $\left(M_{2}(\mathbb{C}), \operatorname{Ad}(\mathfrak{c})\right)$, the subalgebra of real elements is spanned (over the real numbers) by the identity matrix and the matrices $i \sigma_{x}, i \sigma_{y}, i \sigma_{z}$, where

$$
\sigma_{x}:=\left(\begin{array}{ll}
0 & 1 \\
1 & 0
\end{array}\right), \quad \sigma_{y}:=\left(\begin{array}{cc}
0 & -i \\
i & 0
\end{array}\right), \quad \sigma_{z}:=\left(\begin{array}{cc}
1 & 0 \\
0 & -1
\end{array}\right)
$$

are the usual Pauli matrices. This subalgebra is thus isomorphic (as an algebra over the real numbers) to the quaternions $\mathbb{H}$. For this reason, we will denote the real $\mathrm{C}^{*}$-algebra $\left(M_{2}(\mathbb{C}), \operatorname{Ad}(\mathfrak{c})\right)$ by $\mathbb{H}_{\mathbb{C}}$.

2.1.6. Local observables as controlled operators Besides being Hermitian and preserving the CAR form, the Hamiltonians $H$ relevant to our considerations share a further common feature. Namely, they are local operators. By this we mean that $(H \psi)(x)$ depends only on the values $\psi(y)$ for $y$ in a neighbourhood of $x$ whose diameter is bounded independently of $x$. Mathematically, this is captured by the notion of controlled operators defined below.

Definition 6 (Controlled operators). Let $U$ be a finite-dimensional Hilbert space, $D \subseteq$ $\Lambda$ any subset and $O$ a bounded linear operator on $\ell^{2}(D) \otimes U$. Let $O(x, y) \in \operatorname{End}(W)$, for $x, y \in D$, denote the kernel function of $O$, defined by

$$
O(x, y):=\langle x \mid O y\rangle_{\ell^{2}(D)} .
$$

We say that $O$ is controlled if there is a finite constant $R>0$ such that

$$
O(x, y)=0 \quad(\|x-y\|>R) .
$$

The norm closure of the set of controlled operators shall be denoted by $C_{u}^{*}(D, U)$. 
Remark 5. The previous definition is due to Roe [46, Definition 4.22], in a much more general form. The subscript $u$ in $C_{u}^{*}(D, U)$ refers to the word "uniform". Indeed, $C_{u}^{*}(D, U) \cong C_{u}^{*}(D, \mathbb{C}) \otimes \operatorname{End}(U)$ where $C_{u}^{*}(D, \mathbb{C})$ is usually denoted by $C_{u}^{*}(D)$ and called the uniform Roe algebra (of the metric space $D$ ) in the literature. In the context of the topological classification of solid-state systems in the tight-binding approximation, the uniform Roe algebra was first used by Kubota [34].

The following statement is immediate, see [46, Corollary 4.24] for the first part.

Proposition 2. Let $U$ be a finite-dimensional Hilbert space and $D \subseteq \Lambda$ a subset. Then $C_{u}^{*}(D, U)$ is a closed $*$-subalgebra of $\mathscr{L}\left(\ell^{2}(D) \otimes U\right)$ and hence a $C^{*}$-algebra. If $U$ has a real or a quaternionic structure, then $C_{u}^{*}(D, U)$ is invariant under the induced conjugation on $\mathscr{L}\left(\ell^{2}(D) \otimes U\right)$, and thus, a real $C^{*}$-algebra.

Proof. Only the statement about real structure requires proof. But

$$
\bar{O}(x, y)=\overline{O(x, y)} \quad(x, y \in D),
$$

so that controlled operators are invariant under conjugation. Since the latter is an isometry, the assertion follows.

2.1.7. The algebra of bulk observables We now come to the final step of our construction of the bulk algebra. Topological insulators and superconductors occur in solids with a periodic, translation-invariant crystal structure in the bulk. This is already reflected, in the tight-binding approximation, by the choice of the lattice $\Lambda$ of spatial translations leaving the atomic sites invariant. As long as no disorder is present, the smallest $\mathrm{C}^{*}$ algebra containing all free-fermion Hamiltonians of interest on $\mathscr{W}$ is the algebra of $\Lambda$-invariants in $C_{u}^{*}(\Lambda, W)$. In this case, it would be fair to declare it to be the algebra of bulk observables. (Incidentally, the Fourier transform identifies $C_{u}^{*}(\Lambda, W)^{\Lambda}$ with the set of continuous matrix functions $H(k)$ of momenta $k$ on the Brillouin zone $\mathbb{R}^{d} / \Lambda$.)

However, we do not wish to ignore the effects of disorder. Indeed, as we shall show, bulk-boundary correspondence holds in the presence of disorder, both for the strong and the so-called weak invariants. Since disorder breaks the translational invariance at a microscopic level, a major step in building our model is to devise a mathematical counterpart for the macroscopic homogeneity that is nonetheless preserved.

A general way to do this was suggested by Bellissard [6]. Starting from some basic Hamiltonian, he considers all $\Lambda$-translates thereof and the closure $\Omega$ of this translational orbit in the space of operators (for a suitable topology). If the Hamiltonian is macroscopically homogeneous, then, together with its translates, it should [9] be an operator-valued function on $\Omega$ translationally covariant (or equivariant) in that it intertwines the action of the translation group on $\Omega$ and the space of operators.

As there is no way of knowing a priori how to model the space $\Omega$ of disorder configurations, it is common practice to replace this orbit closure by an arbitrary compact Hausdorff space $\Omega$, equipped with a continuous action of the group $\Lambda$. For now, we will leave the choice of $\Omega$ open. We shall discuss a specific choice later, in Sect. 5.3.

Definition 7 (Covariance algebra). Let $\Omega$ be a compact Hausdorff topological space, equipped with a right action of the group $\Lambda$. Let $U$ be a finite-dimensional Hilbert space. Let $u_{x}$, for $x \in \Lambda$, be defined by

$$
\left(u_{x} \psi\right)(y):=\psi(y-x) \quad\left(x, y \in \Lambda, \psi \in \ell^{2}(\Lambda) \otimes U\right)
$$


We define $A^{U}$ to be the set of all maps $O: \Omega \longrightarrow C_{u}^{*}(\Lambda, U)$ that are continuous in the norm topology and covariant (or equivariant) in the sense that

$$
O_{\omega \cdot x}=u_{x}^{*} O_{\omega} u_{x} \quad(x \in \Lambda, \omega \in \Omega) .
$$

In particular, referring to the notation of Paragraph 2.1.4, we set $\mathbb{A}:=A^{W}$ and $\mathrm{A}:=A^{V}$. We sometimes speak of the former as the real case and the latter as the complex case.

Lemma 1. Retain the assumptions of Definition 7. With point-wise operations

$$
\left(O_{1}+\lambda O_{2}\right)_{\omega}:=\left(O_{1}\right)_{\omega}+\lambda\left(O_{2}\right)_{\omega},\left(O^{*}\right)_{\omega}:=\left(O_{\omega}\right)^{*} \quad\left(O, O_{1}, O_{2} \in A^{U}, \lambda \in \mathbb{C}, \omega \in \Omega\right),
$$

and the norm defined by

$$
\|O\|:=\sup _{\omega \in \Omega}\left\|O_{\omega}\right\| \quad\left(O \in A^{U}\right)
$$

the set $A^{U}$ is a $C^{*}$-algebra. If $U$ is real or quaternionic, then $A^{U}$ is a real $C^{*}$-algebra with conjugation and transpose defined by

$$
(\bar{O})_{\omega}:=\overline{O_{\omega}}, \quad\left(O^{\top}\right)_{\omega}:=\left(O_{\omega}\right)^{\top} \quad\left(O \in A^{U}, \omega \in \Omega\right) .
$$

Remark 6 (Magnetic fields). The first one to study the algebra from Definition 7 in connection with solid-state physics was Bellissard, in the context of the quantum Hall effect. (His definition is phrased somewhat differently, using crossed products of $\mathrm{C}^{*}$ algebras; we shall show in Paragraph 2.1.8 that this is equivalent to our definition.) An important feature of the quantum Hall effect considered by Bellissard is the presence of a homogeneous external magnetic field. In building a tight-binding approximation for such a system, one imposes a lattice spacing on the continuum of positions in such a way that exactly one unit of magnetic flux passes through every plaquette. This leads to a quantised magnetic co-cycle $\sigma(x, y)=e^{i(x, \mathbf{B} y) / 2} \in \mathrm{U}(1)$ with $B_{\mu \nu} \in \mathbb{R}$.

Mathematically, there is no obstruction to incorporating such a co-cycle into the definition of the algebra $\mathrm{A}$ in our present context. The situation is different for the case of the real algebra $\mathbb{A}$ : One needs to replace $\sigma$ by the real co-cycle

$$
\Sigma(x, y):=\left(\begin{array}{cc}
e^{\frac{i}{2}(x, \mathbf{B} y)} & 0 \\
0 & e^{-\frac{i}{2}(x, \mathbf{B} y)}
\end{array}\right) .
$$

In addition, this needs to be central, so one has to constrain the units $B_{\mu \nu}$ of magnetic flux per plaquette to integer multiples of $2 \pi$, trivialising the co-cycle. Thus, it is not possible to introduce a homogeneous magnetic field in the case of real symmetries.

This is not a serious limitation: Although magnetic fields can occur in the real symmetry classes $D$ (corresponding to gapped superconductors or superfluids with no symmetries) and $C$ (corresponding to such with fermions of spin $\frac{1}{2}$ and $\mathrm{SU}(2)$ spin-rotation symmetry), they are far from homogeneous; in fact, in the so-called vortex phase they admit magnetic fields as some pattern of magnetic flux tubes.

Furthermore, a quantised magnetic co-cycle, even if it can be treated mathematically, would only be of minor relevance for the physics of the systems we consider. Indeed, in our setting, the lattice $\Lambda$ reflects the periodicity of the pattern of atomic sites. It is therefore fixed by the crystalline structure of the solid; a homogeneous magnetic field quantised perfectly in alignment with the crystalline lattice could only be achieved by 
fine-tuning. Moreover, the absence of a magnetic field should not affect the system's topological features in an essential fashion. For instance, as was discovered by Haldane [22], the Hall conductance in the (anomalous) QHE may still be quantised even when the total magnetic flux per unit plaquette vanishes. See also [43, Corollary 5.7.2].

Referring back to our previous discussion, the space $\Omega$ in Definition 7 and the following lemma is the space of disorder configurations. The covariance condition in Eq. (4) encodes the notion that the bulk observables of interest are macroscopically homogeneous. We shall therefore call the real $\mathrm{C}^{*}$-algebra $\mathbb{A}$ the algebra of bulk observables (or bulk algebra for short). We call the $\mathrm{C}^{*}$-algebra $\mathrm{A}$ the algebra of charge-conserving bulk observables. Note that if $V$ is equipped with a quaternionic structure $T$, then $\mathbb{A}^{\sim}:=A^{V}$ is a real $\mathrm{C}^{*}$-algebra.

Definition 8 (Disordered IQPV). Let $\Omega$ be a compact Hausdorff topological space, equipped with a right action of the lattice $\Lambda$. Then a disordered macroscopically invariant quasi-particle vacuum (or disordered $I Q P V$ ) is a real unitary $J \in \mathbb{A}$ such that $J^{2}=-1$.

In the complex case, a disordered charge-conserving IQPV is defined to be a unitary $J \in \mathrm{A}=A^{V}$ such that $J^{2}=-1$.

The discussion at the end of Paragraph 2.1.3 shows that a disordered charge-conserving IQPV is the same thing as a disordered IQPV $J$ such that $[Q, J]=0$.

2.1.8. The bulk algebra as a crossed product The definition of the bulk algebra $\mathbb{A}$ via controlled operators given above is physically well-motivated. To derive, in Sect. 4, the sequence of $\mathrm{C}^{*}$-algebras that lies at the core of our derivation of the bulk-boundary correspondence, we shall need an alternative description of $\mathbb{A}$ by crossed products. In the following, group elements $g \in G$ are denoted as $\tau_{g}$ when they are to be re-interpreted as generators of a $\mathrm{C}^{*}$-algebra.

Definition 9 (Crossed product $C^{*}$-algebras). Let $G$ be a group and $A$ a $C^{*}$-algebra. An action of $G$ on $A$ is a group homomorphism $\alpha: G \longrightarrow \operatorname{Aut}(A)$; that is, $\alpha_{g}$ is a *-automorphism of $A$ for every $g \in G$, and

$$
\alpha_{g} \circ \alpha_{g^{\prime}}=\alpha_{g g^{\prime}} \quad\left(g, g^{\prime} \in G\right) .
$$

The crossed product $C^{*}$-algebra $A \rtimes_{\alpha} G$ is, by definition, the $\mathrm{C}^{*}$-algebra defined by generators $a \in A$ and $\tau_{g}$, for all $g \in G$, and the following relations: the $\mathrm{C}^{*}$-algebraic relations of $A$, together with

$$
\tau_{g} \tau_{g^{\prime}}=\tau_{g g^{\prime}}, \quad \tau_{g}^{*}=\tau_{g^{-1}}, \quad \tau_{g} a=\alpha_{g}(a) \tau_{g} \quad\left(g, g^{\prime} \in G, a \in A\right) .
$$

A tuple $(\pi, U)$ consisting of a non-degenerate $*$-representation $\pi: A \longrightarrow \mathscr{L}(\mathscr{H})$ and a unitary representation $U: G \longrightarrow \mathrm{U}(\mathscr{H})$ on the same Hilbert space $\mathscr{H}$ is called a covariant pair if

$$
\pi\left(\alpha_{g}(a)\right)=U_{g} \pi(a) U_{g}^{*} \quad(g \in G, a \in A) .
$$

By definition, the $\mathrm{C}^{*}$-algebra $A \rtimes_{\alpha} G$ is universal for covariant pairs. That is, any covariant pair $(\pi, U)$ determines a unique $*$-representation $\pi \rtimes_{\alpha} U: A \rtimes_{\alpha} G \longrightarrow$ $\mathscr{L}(\mathscr{H})$ such that

$$
\left(\pi \rtimes_{\alpha} U\right)(a)=\pi(a), \quad\left(\pi \rtimes_{\alpha} U\right)\left(\tau_{g}\right)=U_{g} \quad(a \in A, g \in G) .
$$


Remark 7. $\mathrm{C}^{*}$-algebras defined by generators and relations are introduced and thoroughly discussed by Blackadar [11].

Twisting co-cycles can be included in the definition of the crossed product, see [16]. The first two relations have to be modified to

$$
\tau_{g} \tau_{g^{\prime}}=\sigma\left(g, g^{\prime}\right) \tau_{g g^{\prime}}, \quad \tau_{g}^{*}=\tau_{g^{-1}}
$$

By [16, Theorem 3.3], the $\mathrm{C}^{*}$-algebra defined by the above relations is the enveloping $C^{*}$-algebra of the Banach $*$-algebra $L^{1}(A, G ; \alpha, \sigma)$ introduced by Busby-Smith.

Before we construct examples of group actions relevant to our applications, let us see how to incorporate real structures.

Definition 10 (Real structures on crossed products). Let $G$ be a group and $\alpha$ an action on $A$, where $(A,-)$ is a real $C^{*}$-algebra. The action is real if for every $g \in G, \alpha_{g}$ is a real $*$-automorphism. In this case, we may extend the conjugation - to one on $A \rtimes_{\alpha} G$ by setting $\overline{\tau_{g}}:=\tau_{g}$ for all $g \in G$. A covariant pair $(\pi, U)$ on the Hilbert space $\mathscr{H}$ is called real if $\mathscr{H}$ is a real Hilbert space, $\pi$ is real, and $U_{g}$ is real for every $g \in G$. The *-representation $\pi \rtimes_{\alpha} U$ induced by a real covariant pair is real.

As before, let $\Omega$ be a compact Hausdorff space, equipped with a right action of $\Lambda$. We define a real action $\alpha$ of $\Lambda$ on $\mathscr{C}(\Omega) \otimes \operatorname{End}(W)$ by

$$
\alpha_{x}(f)(\omega):=f(\omega \cdot x) \quad(x \in \Lambda, f \in \mathscr{C}(\Omega) \otimes \operatorname{End}(W), \omega \in \Omega) .
$$

Here, $\mathscr{C}(\Omega)$ is the $\mathrm{C}^{*}$-algebra of continuous functions on $\Omega$, equipped with complex conjugation. The same formula defines an action $\alpha$ of $\Lambda$ on $\mathscr{C}(\Omega) \otimes \operatorname{End}(V)$.

Theorem 1. The crossed product $C^{*}$-algebra $(\mathscr{C}(\Omega) \otimes \operatorname{End}(W)) \rtimes_{\alpha} \Lambda$ is isomorphic to $\mathbb{A}$ as a real $C^{*}$-algebra. Similarly, $\mathrm{A} \cong(\mathscr{C}(\Omega) \otimes \operatorname{End}(V)) \rtimes_{\alpha} \Lambda$ as $C^{*}$-algebras.

Remark 8. Although our definition of the algebras $\mathbb{A}$ and $\mathrm{A}$ is equivalent to a crossed product, we believe that it is more natural to introduce these algebras in the fashion we have chosen. We are especially motivated to do so by our quest to develop the mathematical model from its foundations in many-body quantum mechanics, thus providing it with a sound physical meaning. Another benefit of this approach is that it can be extended to include a group $G$ of spatial symmetries. In this case, $\Lambda$ is the full set of atomic sites, invariant under the space group $G$. In this case, the resulting algebra is a corner of the crossed product $(\mathscr{C}(\Omega) \otimes \operatorname{End}(W)) \rtimes G$. This corner is Morita equivalent to the crossed product itself under the assumptions that at every point of $\Lambda$, the on-site representations of the isotropy group of the $G$-action contain all irreducible representations.

The statement of Theorem 1 is well-known to experts and is implicit in the literature, see for example [7, Proposition 5], although full proofs are not readily available. Therefore, and because we need to refer back to it below, we give a detailed proof.

Proof of Theorem 1. We prove only the statement for $\mathbb{A}$; the other case is almost literally the same. Abbreviate $\tilde{A}:=\mathscr{C}(\Omega) \otimes \operatorname{End}(W)$. We may equip $\Omega$ with a fully supported Borel probability measure $\mathbb{P}$ (say), so that $\mathbb{A}$ is realised in $\mathscr{L}\left(L^{2}(\Omega, \mathbb{P}) \otimes \mathscr{W}\right)$. Define

$$
\left.\begin{array}{rl}
(\pi(f) \psi)(\omega, y) & :=f(\omega \cdot(-y)) \psi(\omega, y) \\
\left(R_{x} \psi\right)(\omega, y) & :=\psi(\omega, y+x)
\end{array}\right\} \quad\left(\psi \in L^{2}(\Omega, \mathbb{P}) \otimes \mathscr{W}, \omega \in \Omega, x, y \in \Lambda\right) .
$$


Then by [42, Section 7.7.1], $(\pi, R)$ is a covariant pair that is manifestly real. It thus defines a real $*$-representation $\Phi:=\pi \rtimes_{\alpha} R$ of $\tilde{A} \rtimes_{\alpha} \Lambda$. Since $\Lambda$ is amenable, [42, Theorems 7.7.5 and 7.7.7] shows that $\Phi$ is faithful.

We claim that the image of $\Phi$ lies in $\mathbb{A}$. By the definitions, $\Phi\left(\tau_{x}\right)_{\omega}$ and $\Phi(f)_{\omega}$ are controlled for any $x \in \Lambda, f \in \mathscr{C}(\Omega) \otimes \operatorname{End}(W)$, and $\omega \in \Omega$. Hence, $\Phi(a)_{\omega} \in$ $C_{u}^{*}(\Lambda, W)$ for any $a \in \tilde{A} \rtimes_{\alpha} \Lambda$. To check the covariance condition, we compute

$$
u_{g}^{*} \Phi\left(\tau_{x}\right)_{\omega} u_{g}=u_{-g} u_{-x} u_{g}=u_{-x}=R_{x}=\Phi\left(\tau_{x}\right)_{\omega \cdot g} \quad(x, g \in \Lambda, \omega \in \Omega) .
$$

Similarly, we have

$$
\left(u_{g}\right)^{*} \Phi(f)_{\omega} u_{g}=\sum_{x \in \Lambda} f(\omega \cdot x)|x-g\rangle\langle x-g|=\Phi(f)_{\omega \cdot g} \quad(f \in \tilde{A}, g \in \Lambda, \omega \in \Omega) .
$$

This shows that $\operatorname{im} \Phi \subseteq \mathbb{A}$. We have to prove the equality.

Let $c_{0}(\Lambda)$ denote the $\mathrm{C}^{*}$-algebra of null sequences indexed by $\Lambda$. Because $\Lambda$ is an amenable group, there is an approximate unit $\left(\phi_{n}\right)_{n \in \mathbb{N}}$ for $c_{0}(\Lambda)$ consisting of finitely supported functions of positive type [46, Lemma 11.19]. We may suppose that $\phi_{n}(0)=1$. For every $n$, there is a finite-dimensional Hilbert space $\mathscr{H}_{n}$, a unitary representation $\pi_{n}$ of $\Lambda$ on $\mathscr{H}_{n}$, and a unit vector $\xi_{n} \in \mathscr{H}_{n}$ such that $\phi_{n}(x)=\left\langle\xi_{n} \mid \pi_{n}(x) \xi_{n}\right\rangle$ for $x \in \Lambda$.

Let $\left(\xi_{n}^{i}\right)$ be an orthonormal basis of $\mathscr{H}_{n}$. Set

$$
f_{n}^{i}(x):=\left\langle\xi_{n}^{i} \mid \pi_{n}(x) \xi_{n}\right\rangle \quad(x \in \Lambda),
$$

so that $f_{n}^{i}$ is bounded on $\Lambda$. For $T \in \mathscr{L}(\mathscr{W})$, we define

$$
S_{n}(T):=\sum_{i} M_{f_{n}^{i}}^{*} T M_{f_{n}^{i}} \in \mathscr{L}(\mathscr{W})
$$

where $M_{f}$ is multiplication by $f$. Observe that

$$
\begin{aligned}
S_{n}(T) & =\sum_{x, y} \sum_{i}\left\langle\pi_{n}(x) \xi_{n} \mid \xi_{n}^{i}\right\rangle\left\langle\xi_{n}^{i} \mid \pi_{n}(y) \xi_{n}\right\rangle T(x, y)|x\rangle\langle y| \\
& =\sum_{x, y} \phi_{n}(y-x) T(x, y)|x\rangle\langle y| .
\end{aligned}
$$

In particular, $S_{n}(1)=1$, as $\phi_{n}(0)=1$. It is clear that $S_{n}$ is completely positive, $c f$. [46, Lemma 11.17], and in particular positive. Hence $\left\|S_{n}\right\| \leqslant\left\|S_{n}(1)\right\|=1$, that is, $S_{n}$ is a contraction. Since $S_{n}$ leaves controlled operators invariant, it leaves $C_{u}^{*}(\Lambda, W)$ invariant.

Let $T \in \mathbb{A}$. We set $S_{n}(T)_{\omega}:=S_{n}\left(T_{\omega}\right)$. Since $S_{n}$ commutes with $u_{x}$ for any $x$, this defines $S_{n}(T) \in \mathbb{A}$. Let $\varepsilon>0$ be arbitrary. Because $\Omega$ is compact, there exist a finite $J$, points $\omega_{j} \in \Omega, j \in J$, and open neighbourhoods $U_{j} \subseteq \Omega$ of $\omega_{j}$ covering $\Omega$ such that

$$
\left\|T_{\omega}-T_{\omega_{j}}\right\| \leqslant \frac{\varepsilon}{6} \quad\left(\omega \in U_{j}, j \in J\right)
$$

There exist a constant $R>0$ and $R$-controlled operators $T_{j} \in C_{u}^{*}(\Lambda, W)$ such that

$$
\left\|T_{\omega_{j}}-T_{j}\right\| \leqslant \frac{\varepsilon}{6} \quad(j \in J) .
$$


By [46, Lemma 4.27], there is a constant $C_{R}>0$ depending only on $R$ such that $\left\|T_{j}-S_{n}\left(T_{j}\right)\right\| \leqslant C_{R} \cdot \sup _{\|x\| \leqslant R}\left|1-\phi_{n}(x)\right| \cdot \sup _{\|x-y\| \leqslant R}\left\|T_{j}(x, y)\right\| \quad(n \in \mathbb{N}, j \in J)$.

Since $\phi_{n}$ converges to 1 uniformly on $\{\|\cdot\| \leqslant R\}$, there is an $N \in \mathbb{N}$ such that for all $n \geqslant N$ and all $j \in J$, the right-hand side is $\leqslant \frac{\varepsilon}{3}$.

If now $n \geqslant N$ and $\omega \in \Omega$, then there is some $j$ such that $\omega \in U_{j}$. It follows that

$$
\begin{aligned}
\left\|T_{\omega}-S_{n}\left(T_{\omega}\right)\right\| \leqslant & \left\|T_{\omega}-T_{\omega_{j}}\right\|+\left\|T_{\omega_{j}}-T_{i}\right\|+\left\|T_{j}-S_{n}\left(T_{j}\right)\right\| \\
& +\left\|S_{n}\left(T_{j}-T_{\omega_{j}}\right)\right\|+\left\|S_{n}\left(T_{\omega_{j}}-T_{\omega}\right)\right\| \\
\leqslant & 2\left\|T_{\omega}-T_{\omega_{j}}\right\|+2\left\|T_{\omega_{j}}-T_{i}\right\|+\left\|T_{j}-S_{n}\left(T_{j}\right)\right\| \leqslant \frac{4 \varepsilon}{6}+\frac{\varepsilon}{3}=\varepsilon,
\end{aligned}
$$

so $S_{n}(T)$ converges to $T$ in $\mathbb{A}$. Since im $\Phi$ is closed, it suffices to show that $S_{n}(T) \in \operatorname{im} \Phi$.

For any $n \in \mathbb{N}$ and $y \in \operatorname{supp}\left(\phi_{n}\right)$, define $f_{n, y} \in \mathscr{C}(\Omega) \otimes \operatorname{End}(W)$ by

$$
f_{n, y}(\omega):=T_{\omega}(0, y) \quad(\omega \in \Omega) .
$$

Then we compute

$$
\begin{aligned}
\operatorname{im} \Phi \ni \sum_{\phi_{n}(y) \neq 0} \phi_{n}(y) \pi\left(f_{n, y}\right) R_{-y} & =\sum_{\phi_{n}(y) \neq 0, x \in \Lambda} \phi_{n}(y) T_{\omega \cdot x}(0, y)|x\rangle\langle x| R_{-y} \\
& =\sum_{\phi_{n}(y-x) \neq 0} \phi_{n}(y-x) T_{\omega}(x, y)|x\rangle\langle y|=S_{n}(T) .
\end{aligned}
$$

Thus, $\Phi$ is surjective. As we already know that $\Phi$ is injective, this proves the theorem.

\section{Symmetries, Pseudo-Symmetries, and Bulk Invariants}

3.1. Quasi-particle vacua with symmetries. Our mission is to study symmetry-protected topological (or SPT) phases of disordered free fermions. While such phases constitute a rather large class, a manageable and especially interesting subclass is the one given by the classification scheme of the Tenfold Way [24]. In that scheme, one starts from the Nambu space of fields equipped with the CAR form and at most two distinguished antiunitary operations, namely time-reversal and/or particle-hole symmetry. The setting also allows for an arbitrary group $G_{0}$ of unitary symmetries. The classification statement of the Tenfold Way then is that, upon projecting Nambu space to any isotypical component of irreducible $G_{0}$-representations, one invariably ends up with a reduced space that is of one of 10 types known as the AZ (or CAZ) classes [1]. The early work [33,48] on free-fermion SPT phases focused on precisely those 10 types or symmetry classes.

In trying to set up a concise description of all Tenfold-Way symmetry classes in a unified $\mathrm{C}^{*}$-algebraic framework, one faces the complication that several real structures co-exist. Indeed, there is the Majorana or $\gamma$-real structure and one may also have the anti-linear operations of time-reversal and/or particle-hole symmetry in play (see also the discussion in Paragraph 3.1.5 and following). How can one then avoid a cumbersome case-by-case treatment? It was Kitaev [33] who first put forth the good scheme to use: adopting the universal real structure offered by the real space of Majorana fields, he implements the anti-linear symmetries as part of a Clifford algebra of complex linear (!) generators. That scheme was worked out in systematic detail by Kennedy-Zirnbauer [32], and our first step here is to adapt their Bloch-bundle picture to the $\mathrm{C}^{*}$-algebraic setting for systems with disorder. 
3.1.1. On-site symmetries Our bare algebraic framework is enriched by symmetry operations that act on the Nambu space $\mathscr{W}=\ell^{2}(\Lambda) \otimes W$ or rather, as on-site symmetries, on $W$. These operations are linear or anti-linear endomorphisms of $W$ that, if present, constrain our Hamiltonians. With the exception of particle-hole symmetry, all of them are initially defined on the single-particle Hilbert space $\mathscr{V}=\ell^{2}(\Lambda) \otimes V$, or actually, on the on-site Hilbert space $V$. Given that origin, they all turn into operators on $W$ by a canonical procedure: second quantisation takes an operator on $\mathscr{V}$ to an operator on the Fock space $\bigwedge\left(\mathscr{V}_{+} \oplus \mathscr{V}_{-}^{*}\right)$. The latter then determines a ("de-quantised") operator on $\mathscr{W}$ by the commutator or adjoint action, $c f$. Sect. 2.1.

The first symmetry we wish to consider is time reversal, $T$. On electrons (or any other fundamental fermions), this is an anti-unitary operator such that $T^{2}=-1$. Put differently, time reversal is simply a quaternionic structure on $V$, see Definition 5 . The afore-mentioned functor of second quantisation followed by de-quantisation extends $T$ to a real anti-linear endomorphism of $W$ by $\left.T\right|_{V^{*}}:=h T h^{-1}$.

The next set of symmetries that occur on $V$ are related to the spin rotation generators $S_{1}, S_{2}, S_{3}$. For electrons (or any other fundamental fermions) carrying spin $\frac{1}{2}$ these satisfy the relations

$$
S_{1} S_{2}=-S_{2} S_{1}=i S_{3}, \quad S_{\mu}^{2}=1 \quad(\mu=1,2,3) .
$$

They anti-commute with $T$ (physically speaking, this is because the operation of inverting the time direction makes any motion, also that of spin, run backwards). The operators representing spin rotations are obtained by exponentiation: $g=\exp \left(i \sum \omega_{\mu} S_{\mu}\right) \in$ $\mathrm{SU}(2)=\operatorname{Spin}(3)$, with real parameters $\omega_{\mu}$. To generate SU(2) over the real numbers, we set $j_{\mu}:=i S_{\mu}$, so that

$$
j_{1} j_{2}=-j_{2} j_{1}=-j_{3}, \quad j_{\mu}^{2}=-1 \quad(\mu=1,2,3) .
$$

Overriding physics conventions, we still refer to the $j_{\mu}$ as spin rotation generators. Our quantisation functor yields $\left.j_{\mu}\right|_{V^{*}}:=h j_{\mu} h^{-1}$. Note that the $j_{\mu}$ commute with $T$.

There are two remaining symmetries in our picture. The first of them is $i Q$, which we met earlier as the charge operator $Q=i^{-1} d \rho(i \mathscr{Q})$. The $\gamma$-real operator $i Q$ generates the U(1) symmetry group underlying the law of charge conservation. Recall our above observation, in Paragraph 2.1.3, that an operator on $W$ commutes with $Q$ if and only if it leaves $V$ and $V^{*}$ invariant. It follows that $T$ anti-commutes with $i Q$, and that the $j_{\mu}$, $\mu=1,2,3$, commute with $i Q$.

Finally, we consider the particle-hole symmetry $C$ on $W$. Recall from Sect. 2.1 that the Fock space $\bigwedge$ of the many-particle system was built from the Hilbert space $\mathscr{V}_{+} \oplus \mathscr{V}_{-}^{*}$, where $\mathscr{V}_{+}=\ell^{2}(\Lambda) \otimes V_{+}$and $\mathscr{V}_{-}=\ell^{2}(\Lambda) \otimes V_{-}$are the subspaces harbouring the conduction and the valence states, respectively. A special situation that may occur is that the conduction and valence spaces are in bijection via an operator

$$
S: \mathscr{V}_{+} \oplus \mathscr{V}_{-} \longrightarrow \mathscr{V}_{-} \oplus \mathscr{V}_{+}
$$

which exchanges the conduction and valence states and anti-commutes with the firstquantised Hamiltonian $\mathrm{H}$. In principle, $S$ may be linear or anti-linear. For example, for Dirac fermions one knows the operation of charge conjugation $\psi \longmapsto i \gamma^{2} \bar{\psi}$ (using the standard physics notation for $\gamma$-matrices, not to be confused with our choice of real structure). Charge conjugation is complex anti-linear in first quantisation (and, perhaps confusingly, complex linear after second quantisation) and anti-commutes with the Dirac Hamiltonian in the absence of electromagnetic fields. Yet, in the condensed 
matter physics of non-relativistic electrons, there is no such thing as the relativistic charge conjugation operator. However, what may exist in that setting is a sub-lattice (or "chiral") operation $S: \psi_{A}+\psi_{B} \longmapsto \psi_{A}-\psi_{B}$, which is complex linear.

In keeping with our use of the tight-binding approximation, we assume the operator $S$ to be realised on $V=V_{+} \oplus V_{-}$, so that $S \in \mathrm{U}(V), S^{2}=1,[S, T]=0$, and $\left[S, j_{\mu}\right]=0$, $\mu=1,2,3$. As before, $S$ extends to a real linear endomorphism of $W$ by $\left.S\right|_{V^{*}}:=h S h^{-1}$. In particular, $[S, Q]=0$ for this extension. The particle-hole symmetry $C$ is then defined by $C:=\gamma S$; indeed, if the free-fermion Hamiltonian $H=-\gamma H \gamma$ anti-commutes with the sub-lattice operation $S$, then $C=\gamma S$ commutes with $H$ and is thus a true physical symmetry. (Moreover, the many-particle Fock vacuum $\bigwedge^{0}\left(\mathscr{V}_{+} \otimes \mathscr{V}_{-}^{*}\right)$ is particle-hole symmetric.)

It should be stressed here that our notion of particle-hole symmetry continues to make sense in the realm of interacting systems beyond the free-fermion approximation. As a matter of fact, the anti-linear operator $C: \mathscr{W} \rightarrow \mathscr{W}$ lifts to a real or quaternionic structure on the many-particle Fock space $\bigwedge$, and the latter operation induces a real structure on the algebra of bounded many-body operators. An important example of an interacting Hamiltonian with particle-hole symmetry is that of the Hubbard model.

3.1.2. Symmetry classes We shall say that a Hermitian and $\gamma$-imaginary Hamiltonian $H \in \mathscr{L}(\mathscr{W})$ is in the (real) symmetry class $s$ (where $s=0, \ldots, 7$ ) if it commutes with the symmetries indicated in Table 1 . There, the symbol U(1) ${ }_{c}$ stands for the U(1) group generated by the charge operator $i Q$, and $\mathrm{SU}(2)_{s}$ denotes the $\mathrm{SU}(2)$ group of spin rotations generated by the $j_{\mu}$. We say that $H$ is in the complex symmetry class $s$ (where $s=0,1)$ if it commutes with the symmetries indicated in Table 2 .

Note that if the Hamiltonian preserves charge, i.e. commutes with $Q$, then we may reduce it to its first-quantised form on $\mathscr{V}$.

Although our symmetry-class tables look different from those widely cited and used in the literature $[33,48]$, they lead to mathematically equivalent results. What we follow here is the assessment of Kennedy-Zirnbauer [32] that, at the level of setting the foundations, all declarations of symmetry should conform to the principle that true physical symmetries commute with the Hamiltonian (never do they anti-commute). One benefit from adhering to that strict notion of symmetry is that our framework directly extends to systems with electron-electron interactions. Indeed, all our symmetries $X$ make im-

Table 1. Symmetry classes

\begin{tabular}{lllll}
\hline$s$ & Class & Symmetry group & Generators & Comments \\
\hline 0 & $D$ & Trivial & - & Time reversal \\
1 & $D$ III & $\mathbb{Z}_{4}$ & $T$ & Charge \\
2 & $A$ II & $\mathbb{Z}_{4} \ltimes \mathrm{U}(1)_{c}$ & $T, i Q$ & Particle-hole \\
3 & $C$ II & $\mathbb{Z}_{4} \ltimes \mathrm{U}(1)_{c} \times \mathbb{Z}_{2} . i Q, C$ & Spin \\
4 & $C$ & $\mathrm{SU}(2)_{s}$ & $j_{\mu}$ & \\
5 & $C \mathrm{I}$ & $\mathrm{SU}(2)_{s} \times \mathbb{Z}_{4}$ & $j_{\mu}, T$ & \\
6 & $A \mathrm{I}$ & $\mathrm{SU}(2)_{s} \times\left(\mathbb{Z}_{4} \ltimes \mathrm{U}(1)_{c}\right)$ & $j_{\mu}, T, i Q$ & \\
7 & $B D \mathrm{I}$ & $\mathrm{SU}(2)_{s} \times \mathbb{Z}_{4} \ltimes \mathrm{U}(1)_{c} \times \mathbb{Z}_{2}$ & $j_{\mu}, T, i Q, C$ & \\
\hline
\end{tabular}

Table 2. Complex symmetry classes

\begin{tabular}{lllll}
\hline$s$ & Class & Symmetry group & Generators & Comments \\
\hline 0 & $A$ & $\mathrm{U}(1)_{c}$ & $i Q$ & Charge \\
1 & $A$ III & $\mathrm{U}(1)_{c} \times \mathbb{Z}_{2}$ & $i Q, C$ & Particle-hole \\
\hline
\end{tabular}


mediate sense at the interacting many-particle level (actually, as we have indicated, they descend from their second-quantised ancestors $\mathscr{X}$ by the transfer to Nambu space), and they constrain the symmetry-allowed Hamiltonians by $\mathscr{X} \mathscr{H}=+\mathscr{H} \mathscr{X}$ (never by $\mathscr{X} \mathscr{H}=-\mathscr{H} \mathscr{X}$ ). The relation of the present scheme to that of Schnyder-RyuFurusaki-Ludwig [48] is reviewed in the appendix to this section.

Remark 9. The terminology for symmetry classes was introduced by Altland-Zirnbauer [1] in their work on disordered free fermions in mesoscopic systems, especially superconductors. The labels $(D, D$ III, etc.) used in that context refer to the Cartan-Killing classification of Riemannian symmetric spaces. Let us emphasise that we use the (C)AZ terminology precisely as intended by its pioneers, namely for the case with disorder, and please be warned that much of the recent literature has taken to (ab-)using that terminology for translation-invariant systems without disorder.

3.1.3. Pseudo-symmetries Some of the physical symmetries we consider are linear, while others are anti-linear. Given a symmetry $g$ that commutes with the gapped Hamiltonian $H$, whether $g$ commutes or anti-commutes with the corresponding IQPV $J=$ $-i \operatorname{sign}(H)$ depends on whether $g$ is linear or anti-linear. Since we are interested primarily in ground states rather than Hamiltonians, our description of symmetry classes of IQPV would not be smooth if done in terms of the original symmetries. This situation is resolved by the concept of pseudo-symmetries advertised by Kennedy-Zirnbauer [32].

Following Kitaev [33] they organise the symmetry classes of translationally invariant free-fermion ground states by assigning, to each of the sets of symmetries in Table 1, which commute with $H$, a set of $s$ operators $J_{1}, \ldots, J_{s}$, called pseudo-symmetries, which anti-commute with the IQPV $J$. Here, $s$ denotes the numerical label in the table. Besides anti-commuting with $J$, the pseudo-symmetries define a real $*$-representation of a Clifford algebra. To make this precise, we recall the following standard definition.

Definition 11 (Clifford algebras). Let $\mathbb{C} \ell_{r, s}$ be the universal $\mathrm{C}^{*}$-algebra with unitary generators $k_{1}, \ldots, k_{r}, j_{1}, \ldots, j_{s}$ and relations

$$
\left.\begin{array}{l}
k_{a} k_{b}+k_{b} k_{a}=2 \delta_{a b} \\
j_{\alpha} j_{\beta}+j_{\beta} j_{\alpha}=-2 \delta_{\alpha \beta} \\
k_{a} j_{\alpha}+j_{\alpha} k_{a}=0
\end{array}\right\} \quad(a, b=1, \ldots, r, \alpha, \beta=1, \ldots, s) .
$$

This is the familiar Clifford algebra with $r$ positive and $s$ negative generators.

We define an anti-linear involution ${ }^{-}$on $\mathbb{C} \ell_{r, s}$ by declaring all of the generators $k_{a}, j_{\alpha}$ to be fixed by it. The resulting real $\mathrm{C}^{*}$-algebra $\left(\mathbb{C} \ell_{r, s},{ }^{-}\right)$will be denoted by $C \ell_{r, s}$.

Remark 10. With the terminology introduced in Definition 11, any collection of real unitaries $K_{1}, \ldots, K_{r}, J_{1}, \ldots, J_{s}$ on $W$ such that

$$
\left.\begin{array}{l}
K_{a} K_{b}+K_{b} K_{a}=2 \delta_{a b} \\
J_{\alpha} J_{\beta}+J_{\beta} J_{\alpha}=-2 \delta_{\alpha \beta} \\
K_{a} J_{\alpha}+J_{\alpha} K_{a}=0
\end{array}\right\} \quad(a, b=1, \ldots, r, \alpha, \beta=1, \ldots, s)
$$

determines a unique real $*$-morphism $C \ell_{r, s} \longrightarrow \operatorname{End}(W) v i a k_{a} \longmapsto K_{a}$ and $j_{a} \longmapsto J_{\alpha}$, and vice versa. Similarly, a set of unitaries on $V$ satisfying the relations in Equation (6) amount to the same data as a $*$-morphism $\mathbb{C} \ell_{r, s} \longrightarrow \operatorname{End}(V)$. 
Let us now review the Kennedy-Zirnbauer construction briefly. When $0 \leqslant s \leqslant 3$, we may define a set $J_{1}, \ldots, J_{s}$ of anti-commuting real unitaries on $W$ by

$$
J_{1}:=J_{T}:=\gamma T, \quad J_{2}:=J_{Q}:=i \gamma Q T, \quad J_{3}:=J_{C}:=i \gamma Q C .
$$

When $s \geqslant 4$, we amplify $W$ to $W \otimes \mathbb{C}^{2}$, giving $\mathbb{C}^{2}$ its canonical real structure $\mathbb{R}^{2} \subset \mathbb{C}^{2}$, and we define

$$
\begin{aligned}
J_{\mu} & :=j_{\mu} \otimes \sigma_{z} \quad(\mu=1, \ldots, 3), \\
J_{4} & :=1 \otimes i \sigma_{y}, \quad J_{5}:=J_{T} \otimes \sigma_{x}, \quad J_{6}:=J_{Q} \otimes \sigma_{x}, \quad J_{7}:=J_{C} \otimes \sigma_{x} .
\end{aligned}
$$

In particular, this redefines $J_{1}, \ldots, J_{3}$. We obtain a set $J_{1}, \ldots, J_{s}$ of $s$ anti-commuting real unitaries on $W \otimes \mathbb{C}^{2}$.

For $0 \leqslant s \leqslant 3, H$ commutes with the corresponding symmetries in Table 1 if and only if $J$ anti-commutes with $J_{1}, \ldots, J_{s}$. For $s \geqslant 4, H$ commutes with the corresponding symmetries if and only if $J \otimes \sigma_{x}$ anti-commutes with $J_{1}, \ldots, J_{s}$. Therefore, and motivated by [32, Definition 2.4], we pose the following definition.

Definition 12 (Disordered IQPV with symmetries). A disordered IQPV of symmetry index $(r, s)$ (or simply of index $(r, s))$ is a tuple $\left(J ; K_{1}, \ldots, K_{r}, J_{1}, \ldots, J_{s}\right)$ where $J \in \mathbb{A}$ is a disordered IQPV and $\left(K_{1}, \ldots, K_{r}, J_{1}, \ldots, J_{s}\right)$ define a real $*$-representation $\phi: C \ell_{r, s} \longrightarrow \operatorname{End}(W)$ such that

$$
J K_{a}+K_{a} J=J J_{\alpha}+J_{\alpha} J=0 \quad(a=1, \ldots, r, \alpha=1, \ldots, s) .
$$

If $r=0$ and $0 \leqslant s \leqslant 7$, then we also call this a disordered IQPV of symmetry class $s$. We shall also refer to it by the Cartan-Killing label in Table 1. (For instance, " $J$ is a disordered IQPV of class $D$.") We call the $J_{\alpha}$ negative and the $K_{a}$ positive pseudosymmetries of $J$.

Similarly, a disordered IQPV of complex symmetry index $(r, s)$ consists of a disordered charge-preserving IQPV $J \in \mathrm{A}$ and $K_{a}, J_{\alpha} \in \operatorname{End}(V)$ defining a $*$-representation of $\mathbb{C} \ell_{r, s}$ such that Eq. (10) holds; we call these $K_{a}, J_{\alpha}$ complex pseudo-symmetries. For $r=0$ and $s=0,1$, we say that $\left(J ; J_{\alpha}\right)$ are in complex symmetry class $s$.

Remark 11. In view of the correspondence between operators $K_{a}, J_{\alpha}$ and Clifford representations $\phi$, we will interchangeably write a disordered IQPV of a given (complex) symmetry index as $(J ; \phi)$ where $\phi$ is the $*$-morphism representing the (complex) pseudosymmetries.

The discussion at the end of Paragraph 2.1.3 shows that a disordered IQPV of complex symmetry index $(r, s)$ is the same as a disordered IQPV of symmetry index $(r, s)$ with the additional property that both $J$ and the $K_{a}, J_{\alpha}$ commute with $Q$.

3.1.4. Background on Clifford algebras In this section, we collect some background results on Clifford algebras. These will explain several delicate but important points in our classification scheme for IQPV with pseudo-symmetries.

One background fact is the mod 8 periodicity of Clifford algebras (resp. the mod 2 periodicity of complex Clifford algebras); this explains why we consider "real" symmetry classes $s$ where $s=0, \ldots, 7$ (resp. "complex" symmetry classes indexed by $s=$ $0,1)$. Another one is the quaternion periodicity of Clifford algebras, which explains the symmetry between the "real" cases $0 \leqslant s \leqslant 3$ and $4 \leqslant s \leqslant 7$. Yet another point is that up to equivalence and multiplicity, the symmetry class labelled by $s$ is determined by $s$ and does not depend on the choice of the operators $J_{\alpha}$. This is related to the classification 
of Clifford representations. Finally, we will review the Clifford $(1,1)$ periodicity, which is not only at the basis of the other periodicity results, but also fundamental for our construction of boundary invariants. For the remaining discussion, we introduce the notion of a grading.

Definition 13 (Graded real $\mathrm{C}^{*}$-algebras). A grading on a $*$-algebra $A$ is a decomposition $A=A_{\overline{0}} \oplus A_{\overline{1}}$, where $\mathbb{Z} / 2 \mathbb{Z}=\{\overline{0}, \overline{1}\}$, into closed subspaces such that

$$
A_{i} \cdot A_{j} \subseteq A_{i+j}, *\left(A_{i}\right) \subseteq A_{i} \quad(i, j \in \mathbb{Z} / 2 \mathbb{Z}) .
$$

When equipped with a grading, $A$ is called a graded $*$-algebra. Elements of $A_{\overline{0}}$ are called even, while those of $A_{\overline{1}}$ are called odd. Non-zero elements $a$ in either of these summands are called homogeneous; in this case, $|a|$ denotes the parity, $\overline{0}$ if $a$ is even and $\overline{1}$ if $a$ is odd. If $A$ is a $C^{*}$-algebra, then we suppose in addition that $A_{i}, i=\overline{0}, \overline{1}$, are closed; $A$ is then called a graded $C^{*}$-algebra. A map of graded $*$-algebras is called even if it preserves the grading. If $\left(A,{ }^{-}\right)$is a real $*$-algebra equipped with a grading, then it is called a graded real $*$-algebra if the conjugation is even. A graded real $*$-algebra that is also a graded $C^{*}$-algebra is called a graded real $C^{*}$-algebra.

For graded $*$-algebras $A$ and $B$, the algebraic tensor product $A \odot B$ is graded by

$$
(A \odot B)_{k}:=\bigoplus_{i+j=k} A_{i} \odot B_{j} \quad(k \in \mathbb{Z} / 2 \mathbb{Z}) .
$$

If $A$ and $B$ are real, then so is $A \odot B$. If $A$ and $B$ are graded (real) $C^{*}$-algebras, then the grading on $A \odot B$ extends to the spatial tensor product $A \otimes B$, turning it into a graded (real) $C^{*}$-algebra.

A different object altogether is the graded tensor product. If $A$ and $B$ are graded *-algebras, then the algebraic tensor product $A \odot B$ carries a new algebra structure and involution induced by

$$
(a \otimes b) \cdot\left(a^{\prime} \otimes b^{\prime}\right):=(-1)^{\left|a^{\prime}\right||b|} a a^{\prime} \otimes b b^{\prime}, \quad(a \otimes b)^{*}=(-1)^{|a||b|} a^{*} \otimes b^{*},
$$

for homogeneous $a, a^{\prime} \in A, b, b^{\prime} \in B$, defining the graded algebraic tensor product $A \widehat{\odot} B$, not isomorphic to $A \odot B$ in general. It is real if so are $A$ and $B$. If $A$ and $B$ are graded (real) $C^{*}$-algebras, then there is a natural $C^{*}$-norm on $A \widehat{\odot} B$. By completing $A \widehat{\odot} B$ with respect to that norm, one obtains a graded (real) $C^{*}$-algebra $A \widehat{\otimes} B[10$, $\S 14.4]$ called the graded tensor product. We will denote the image of an elementary tensor $a \otimes b$ in $A \widehat{\otimes} B$ by the same symbol $a \otimes b$.

Any (real) $C^{*}$-algebra can be considered as a graded (real) $C^{*}$-algebra with the trivial grading, in which every element is even. In particular, we will usually consider the matrix algebras $M_{n}(\mathbb{C})$ as trivially graded and with the component-wise conjugation. The real $\mathrm{C}^{*}$-algebra $\mathbb{H}_{\mathbb{C}}$ will also be considered as ungraded. On the Clifford algebra $C \ell_{r, s}$, one considers the grading that is determined by letting the generators $k_{a}, j_{\alpha}$ be odd.

We now collect some periodicity results on Clifford algebras, $c f$. [4, §4]. Basic to all of the relations between Clifford algebras explained here is the fact that

$$
C \ell_{p+r, q+s} \cong C \ell_{p, q} \widehat{\otimes} C \ell_{r, s}
$$

as graded real $\mathrm{C}^{*}$-algebras. The following is straightforward. 
Proposition 3 (Clifford $(1,1)$ periodicity). There is an isomorphism of real $C^{*}$-algebras $C \ell_{1,1} \longrightarrow M_{2}(\mathbb{C})$, defined on generators by

$$
k_{1} \longmapsto \sigma_{x}, \quad j_{1} \longmapsto-i \sigma_{y} .
$$

If we grade $M_{2}(\mathbb{C})$ by

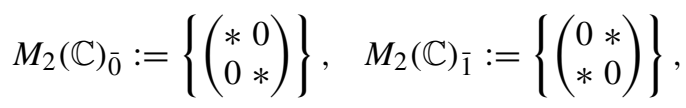

then this isomorphism is even. In particular, we have (1,1)-periodicity:

$$
C \ell_{r+1, s+1} \cong C \ell_{r, s} \widehat{\otimes} M_{2}(\mathbb{C}) .
$$

Recall the following result [4, Proposition 4.2].

Proposition 4 (Atiyah-Bott-Shapiro). There are isomorphisms of graded real $C^{*}$-algebras

$$
C \ell_{0, r+2} \cong C \ell_{0,2} \otimes C \ell_{r, 0}, \quad C \ell_{r+2,0} \cong C \ell_{2,0} \otimes C \ell_{0, r}
$$

induced respectively by

$$
\begin{cases}j_{\alpha} \longmapsto j_{\alpha} \otimes 1, & \text { if } \alpha=1,2, \\ j_{\alpha} \longmapsto j_{1} j_{2} \otimes k_{\alpha-2}, & \text { if } \alpha=3, \ldots, r+2,\end{cases}
$$

and

$$
\begin{cases}k_{a} \longmapsto k_{a} \otimes 1, & \text { if } a=1,2, \\ k_{a} \longmapsto k_{1} k_{2} \otimes j_{a-2}, & \text { if } a=3, \ldots, r+2 .\end{cases}
$$

Note that above, we consider the ungraded tensor product.

From Eq. (3), it is easy to see that $C \ell_{0,2} \cong \mathbb{H}_{\mathbb{C}}$ as ungraded real $C^{*}$-algebras. Similarly, there is an isomorphism $C \ell_{2,0} \cong M_{2}(\mathbb{C})$ of ungraded real $\mathrm{C}^{*}$-algebras, induced by the assignments $k_{1} \longmapsto \sigma_{z}$ and $k_{2} \longmapsto \sigma_{x}$. From this, one deduces $(c f .[4, \S 4])$ isomorphisms of ungraded real $C^{*}$-algebras

$$
C \ell_{0,8} \cong C \ell_{0,2} \otimes C \ell_{0,2} \otimes C \ell_{2,0} \otimes C \ell_{2,0} \cong \mathbb{H}_{\mathbb{C}} \otimes \mathbb{H}_{\mathbb{C}} \otimes M_{4}(\mathbb{C}) .
$$

Since $\mathbb{H}_{\mathbb{C}} \otimes \mathbb{H}_{\mathbb{C}} \cong M_{4}(\mathbb{C})$ as real $C^{*}$-algebras, it follows that

$$
C \ell_{0,8} \cong M_{16}(\mathbb{C})
$$

as ungraded real $\mathrm{C}^{*}$-algebras. A similar argument also shows $C \ell_{8,0} \cong M_{16}(\mathbb{C})$ as ungraded real $C^{*}$-algebras. Together, these isomorphisms are the famous mod 8 periodicity of Clifford algebras [4, Table 1]. Disregarding the real structure, we have $M_{2}(\mathbb{C})=\mathbb{H}_{\mathbb{C}}$; hence, we obtain the isomorphism of (ungraded) $\mathrm{C}^{*}$-algebras

$$
\mathbb{C} \ell_{0,2} \cong \mathbb{C} \ell_{2,0} \cong M_{2}(\mathbb{C}) \text {. }
$$

This is the mod 2 periodicity of complex Clifford algebras. Summarising, the 8 (real) symmetry classes and 2 complex symmetry classes of the Tenfold Way are reflected in the classification of Clifford algebras. 
Table 3. Negative Clifford algebras

\begin{tabular}{ll}
\hline$s$ & $C \ell_{0, s}$ \\
\hline 0 & $\mathbb{C}=\mathbb{R}_{\mathbb{C}}$ \\
1 & $\mathbb{C}_{\mathbb{C}}$ \\
2 & $\mathbb{H}_{\mathbb{C}}$ \\
3 & $\mathbb{H}_{\mathbb{C}} \oplus \mathbb{H}_{\mathbb{C}}$ \\
4 & $M_{2}\left(\mathbb{H}_{\mathbb{C}}\right)$ \\
5 & $M_{4}\left(\mathbb{C}_{\mathbb{C}}\right)$ \\
6 & $M_{8}(\mathbb{C})=M_{8}\left(\mathbb{R}_{\mathbb{C}}\right)$ \\
7 & $M_{8}(\mathbb{C}) \oplus M_{8}(\mathbb{C})$ \\
\hline
\end{tabular}

From the above, we also deduce the following isomorphism:

$$
\begin{aligned}
\mathbb{C} \ell_{0, s+4} & \cong \mathbb{C} \ell_{0,2} \otimes \mathbb{C} \ell_{s+2,0} \cong \mathbb{C} \ell_{0,2} \otimes \mathbb{C} \ell_{2,0} \otimes \mathbb{C} \ell_{0, s} \\
& \cong \mathbb{H}_{\mathbb{C}} \otimes M_{2}(\mathbb{C}) \otimes \mathbb{C} \ell_{0, s} \cong M_{2}\left(\mathbb{H}_{\mathbb{C}}\right) \otimes \mathbb{C} \ell_{0, s},
\end{aligned}
$$

which reflects the symmetry between the symmetry classes indexed by $0 \leqslant s \leqslant 3$ and $4 \leqslant s \leqslant 7$, respectively. Using periodicity, it is possible to compute $C \ell_{0, s}$ in all cases. We reproduce the result from [4, Table 2].

Proposition 5. If $s \not \equiv 3$ (4), then $C \ell_{0, s}$ is simple as a real $C^{*}$-algebra. Moreover, any two finite-dimensional real $*$-representations are unitarily equivalent, provided only that they have the same dimension.

Proof. Simplicity is clear from Table 3. Any finite-dimensional real $*$-representation is the direct sum of irreducible representations, and hence, a multiple of the unique isomorphism class of irreducible real $*$-representation.

The situation for $s=3$ (4) is different in that there exists a central element $\omega=$ $j_{1} \cdots j_{s}$ with $\omega^{2}=+1$. The following statement may be found in [36, Chapter 1 , Propositions 5.9 and 5.10] in a slightly different form.

Proposition 6. Let $s \equiv 3$ (4) and set $\omega:=j_{1} \cdots j_{s}$. There are up to unitary equivalence exactly two irreducible real $*$-representations $\phi$ of $C \ell_{0, s}$, and they are distinguished by $\phi(\omega)=1$ and $\phi(\omega)=-1$, respectively.

Any finite-dimensional real $*$-representation $(W, \phi)$ of $C \ell_{0, s}$ extends to a real $*$ representation of $C \ell_{0, s+1}$ if and only if the eigenvalues \pm 1 of $\phi(\omega)$ have equal multiplicity. In this case, the unitary equivalence class of $(W, \phi)$ and indeed of its extension to $C \ell_{0, s+1}$ is uniquely determined by the dimension of $W$.

Proof. The first part is immediate from Schur's lemma. In particular, the unitary equivalence class of any finite-dimensional real $*$-representation $(W, \phi)$ of $C \ell_{0, s}$ is determined by the multiplicity of the eigenvalues \pm 1 of $\phi(\omega)$.

Assume that in the representation $(W, \phi), W_{ \pm}:=\operatorname{ker}(\phi(\omega) \mp 1)$ have equal dimension. There is an automorphism $\Gamma$ of $C \ell_{0, s}$, defined by

$$
\Gamma\left(j_{\alpha}\right):=-j_{\alpha} \quad(\alpha=1, \ldots, s) .
$$

Then $\Gamma(\omega)=-\omega$. It follows that $\phi_{+} \circ \Gamma$ and $\phi_{-}$are unitarily equivalent, where $\phi_{ \pm}$is the restriction of $\phi$ to $W_{ \pm}$. Hence, there is a real unitary isomorphism $u: W_{+} \longrightarrow W_{-}$ such that

$$
-u \phi_{+}\left(j_{\alpha}\right) u^{*}=u \phi_{+}\left(\Gamma\left(j_{\alpha}\right)\right) u^{*}=\phi_{-}\left(j_{\alpha}\right) \quad(\alpha=1, \ldots, s) .
$$


We may define

$$
\phi\left(j_{s+1}\right):=\left(\begin{array}{cc}
0 & -u^{*} \\
u & 0
\end{array}\right) .
$$

This defines the required extension.

Conversely, let $(W, \phi)$ be a real $*$-representation of $C \ell_{0, s+1}$. Because $\phi\left(\omega_{s}\right)$ anticommutes with $\phi\left(j_{s+1}\right)$, the eigenvalues of $\phi\left(\omega_{s}\right)$ have equal multiplicity. The statement about uniqueness is obvious from Proposition 5, as $s+1 \equiv 0$ (4).

Remark 12. In any given symmetry class labelled by $s$, we shall assume a reference disordered IQPV $J_{\text {ref }} \in \mathbb{A}$ with $s$ negative pseudo-symmetries $J_{1}, \ldots, J_{s} \in \operatorname{End}(W)$. Physically speaking, $J_{\text {ref }}$ represents the "trivial" topological phase. If $J_{\text {ref }}$ is "atomic" or local in the strong sense that $J_{\text {ref }} \in \operatorname{End}(W)$, then by Propositions 5 and 6 , the real Clifford $*$-representation defined by $J_{1}, \ldots, J_{s}$ is determined up to unitary equivalence by $\operatorname{dim} W$.

We now discuss case-by-case what it means for $J$ to be in one of the symmetry classes $s=0, \ldots, 7$, in terms of the physical symmetries introduced in Paragraph 3.1.1. We will in particular use the notation from Eqs. (7-9). However, the construction of both the bulk and the boundary classes, given in Sect. 3.3 and in Sect. 4, will be independent of this case-by-case discussion and will rely solely on the description in terms of pseudosymmetries.

3.1.5. Class DIII, $s=1$ In this case, $V$ carries a time-reversal operator or quaternionic structure $T$ (see Definition 5). That is, $T$ is anti-unitary and $T^{2}=-1$. We assign to $T$ the real unitary $J_{1}=J_{T}=\gamma T$.

Recall that the skew-Hermitian unitary $J$ anti-commutes with the pseudo-symmetry $J_{T}$. Thus $J$ exchanges the two eigenspaces $W_{ \pm}=\operatorname{ker}\left(J_{T} \mp i\right)$ and takes the form

$$
J=\left(\begin{array}{cc}
0 & -u^{*} \\
u & 0
\end{array}\right), \quad u^{*}=u^{-1},
$$

with respect to the decomposition $W=W_{+} \oplus W_{-}$. Now $J=-J^{*}$ is also real or, equivalently, skew (w.r.t. the CAR form). By the algebraic properties of $J_{T}$, the CAR form restricts to a non-degenerate bilinear form $W_{+} \otimes W_{-} \longrightarrow \mathbb{C}$. The resulting identification $W_{-} \cong W_{+}^{*}$ lets us interpret $u \in \operatorname{Hom}\left(W_{+}, W_{-}\right) \cong \operatorname{Hom}\left(W_{+}, W_{+}^{*}\right)$ as a bilinear form on $W_{+}$. The skewness of $J$ then implies skewness of $u$. Thus, any disordered IQPV of class $D$ III corresponds to a unitary and skew-symmetric bilinear form on $W_{+}$. Such forms constitute a symmetric space $\mathrm{U} / \mathrm{Sp}$, which is of Cartan-Killing type $A \mathrm{II}$.

3.1.6. Class AII, $s=2$ Here, in addition to the time reversal $T$, we consider the charge operator $Q \in \operatorname{End}(W)$ and $J_{2}:=J_{Q}:=i J_{T} Q$. The following lemma will help to describe the situation.

Lemma 2. Let $x_{1}, x_{2} \in \operatorname{End}(W) \subseteq \mathbb{A}$ be two anti-commuting real skew-Hermitian unitaries. Then $\left(W^{\sim}, T\right)$, where $W^{\sim}:=\operatorname{ker}\left(x_{2} x_{1}-i\right)$ and $T:=-\gamma x_{2}$, is a quaternionic vector space. There is an isomorphism $(W, \gamma) \cong\left(W^{\sim} \otimes \mathbb{C}^{2}, T \otimes \mathfrak{c}\right)$ of real vector spaces such that under the induced isomorphism of real $C^{*}$-algebras

$$
\mathbb{A} \cong \mathbb{A}^{\sim} \otimes \mathbb{H}_{\mathbb{C}}, \quad \mathbb{A}^{\sim}:=A^{W^{\sim}}
$$


we have $x_{1} \longmapsto 1 \otimes i \sigma_{x}$ and $x_{2} \longmapsto 1 \otimes i \sigma_{y}$. Under the isomorphism, real unitaries $J \in \mathbb{A}$ anti-commuting with $x_{1}$ correspond to operators $y_{1} \otimes i \sigma_{y}+y_{2} \otimes i \sigma_{z}$ where $y_{1}, y_{2} \in \mathbb{A}^{\sim}$ are real Hermitian elements such that

$$
y_{1}^{2}+y_{2}^{2}=1, \quad y_{1} y_{2}=y_{2} y_{1}
$$

Proof. Let $x_{3}:=x_{2} x_{1} \in \operatorname{End}(W)$. Then $x_{3}$ is a real skew-Hermitian unitary that anticommutes with $x_{1}$ and $x_{2}$. Setting $W^{ \pm}:=\operatorname{ker}\left(x_{3} \mp i\right)$, we obtain a splitting $W=$ $W^{+} \oplus W^{-}$with respect to which

$$
x_{1}=\left(\begin{array}{cc}
0 & i u^{*} \\
i u & 0
\end{array}\right), \quad x_{2}=\left(\begin{array}{cc}
0 & u^{*} \\
-u & 0
\end{array}\right), \quad x_{3}=\left(\begin{array}{cc}
i & 0 \\
0 & -i
\end{array}\right) .
$$

The map $u: W^{+} \longrightarrow W^{-}$is a unitary isomorphism, and so is

$$
\phi:=\left(\begin{array}{ll}
1 & 0 \\
0 & u
\end{array}\right): W^{+} \oplus W^{+}=W^{+} \otimes \mathbb{C} \longrightarrow W^{+} \oplus W^{-}
$$

We compute

$$
\phi^{*} x_{1} \phi=1 \otimes i \sigma_{x}, \quad \phi^{*} x_{2} \phi=1 \otimes i \sigma_{y} .
$$

Since the anti-unitary map $\gamma x_{2}$ anti-commutes with $x_{3}$, it leaves $W^{+}$invariant. We let $T:=-\left.\gamma x_{2}\right|_{W^{+}}$. Then $T$ is a quaternionic structure on $W^{\sim}:=W^{+}$and

$$
-u T=-\left.x_{2} \gamma x_{2}\right|_{W^{+}}=-\left.\gamma x_{2}^{2}\right|_{W^{+}}=\left.\gamma\right|_{W^{+}},
$$

so that

$$
\phi(T \otimes \mathfrak{c}) \phi^{*}=\phi\left(\begin{array}{cc}
0 & T \\
-T & 0
\end{array}\right) \phi^{*}=\left(\begin{array}{cc}
0 & T u^{*} \\
-u T & 0
\end{array}\right)=\gamma
$$

This proves the claim.

With the lemma above in hand, we can finish the story as follows. Under the isomorphism $\phi: V \otimes \mathbb{C}^{2} \longrightarrow W$ the pseudo-symmetry $J_{Q}$ corresponds to

$$
\phi^{*} J_{Q} \phi=\mathrm{id}_{V} \otimes i \sigma_{y}
$$

Real unitaries $J \in \mathbb{A}$ anti-commuting with $J_{1}, J_{2}$ correspond under $\mathbb{A} \cong \mathbb{A}^{\sim} \otimes \mathbb{H}_{\mathbb{C}}$ exactly to elements of the form $x \otimes i \sigma_{z}$, where $x \in \mathbb{A}^{\sim}$ is a real Hermitian unitary.

Here, the condition that $x$ be real is equivalent to $T x=x T$. Such elements $x$ constitute a symplectic Grassmann manifold $\mathrm{Sp} /(\mathrm{Sp} \times \mathrm{Sp})$, which is a symmetric space of Cartan-Killing type $C$ II. 
3.1.7. Class $C$ II, $s=3$ Besides preserving the time-reversal and charge symmetries, ground states in this symmetry class are invariant under particle-hole symmetry. We have $J_{3}=J_{C}=i \gamma C Q$. The isomorphism $\phi$ from Lemma 2 gives

$$
\phi^{*} J_{C} \phi=\left(\begin{array}{cc}
i S & 0 \\
0 & -i S
\end{array}\right)=S \otimes i \sigma_{z} .
$$

Here, we apply the decomposition $V=V_{+} \oplus V_{-}$for $V_{ \pm}:=\operatorname{ker}(S \mp 1)$.

Similarly, a skew-Hermitian unitary $J \in \mathbb{A}$ anti-commuting with $J_{1}, J_{2}, J_{3}$ acquires the shape $x \otimes i \sigma_{z}$ where $x$ is a real Hermitian unitary commuting with $T$ and anticommuting with $S$. Thus, $x$ takes the form

$$
x=\left(\begin{array}{cc}
0 & y \\
y^{*} & 0
\end{array}\right)
$$

where $y_{\omega}$ is a unitary symplectic operator for every $\omega \in \Omega$, corresponding to the symmetric space USp of type $C$. In terms of the decomposition $\mathbb{A}^{\sim} \cong \mathbb{A} \approx \otimes M_{2}(\mathbb{C})$, where $\mathbb{A} \approx:=A^{V_{+}}$is equipped by the real structure induced by $\left.T\right|_{V_{+}}, y$ is a real unitary in $\mathbb{A} \approx$.

3.1.8. Classes $s \geqslant 4$ In this situation, we consider real unitaries $J \in \mathbb{A}$ such that $J \otimes \sigma_{x}$ anti-commutes with $J_{1}, \ldots, J_{s}$, defined as in Eqs. (8) and (9). This is equivalent to $J$ anti-commuting with the real spin rotation generators $S_{1}, \ldots, S_{3}$ and with the first $s-4$ elements of the sequence $J_{T}, J_{Q}, J_{C}$. Applying Lemma 2 with $x_{1}=j_{1}$ and $x_{2}=j_{2}$, we obtain

$$
\operatorname{End}(W) \cong \operatorname{End}\left(W^{\sim}\right) \otimes \mathbb{H}_{\mathbb{C}}, \quad \mathbb{A} \cong \mathbb{A}^{\sim} \otimes \mathbb{H}_{\mathbb{C}}
$$

where $W^{\sim}=\operatorname{ker}\left(j_{3}-i\right)=\operatorname{ker}\left(S_{3}-1\right)$ and $\mathbb{A}^{\sim}=A^{W^{\sim}}$. Under the isomorphism,

$$
J_{T} \longmapsto j_{T} \otimes 1, \quad J_{Q} \longmapsto j_{Q} \otimes 1, \quad J_{C} \longmapsto j_{C} \otimes 1,
$$

where $j_{T}, j_{Q}, j_{C} \in \operatorname{End}\left(W^{\sim}\right)$ are anti-commuting real skew-Hermitian unitaries. The operator $J$ corresponds to a real skew-Hermitian unitary $j \in \mathbb{A}^{\sim}$ anti-commuting with the first $s-4$ of $j_{T}, j_{Q}, j_{C}$. In this sense, the symmetry classes for $s \geqslant 4$ reduce to the symmetry classes for $0 \leqslant s \leqslant 3$. The only difference is that the real structure on $\mathbb{A}^{\sim}$ comes from a quaternionic structure on $W^{\sim}$ rather than from a real structure.

3.2. The SRFL scheme. In the literature, symmetries are often organised according to a scheme introduced by Schnyder-Ryu-Furusaki-Ludwig [48], which is different from the approach of Kennedy and Zirnbauer that we follow. We speak of the SRFL scheme. It is based on two anti-unitary endomorphisms $\Theta, \Xi: V \longrightarrow V$ and a unitary operator $\Pi$ proportional to $\Xi \Theta$. The following relations are assumed:

$$
\Theta^{2}= \pm 1, \quad \Xi^{2}= \pm 1, \quad \Pi^{2}=1 .
$$

In the literature, $\Theta$ is called time reversal, $\Xi$ is called particle-hole conjugation, and $\Pi$ the chiral or sub-lattice symmetry. As we have already reserved these expressions for certain physical symmetries with potentially different commutation relations, we will not use this terminology in the present section. 
Table 4. Symmetry classes according to Schnyder-Ryu-Furusaki-Ludwig

\begin{tabular}{llll}
\hline Class & $\Theta$ & $\Xi$ & $\Pi$ \\
\hline$A$ & 0 & 0 & 0 \\
$A$ III & 0 & 0 & 1 \\
$D$ & 0 & 1 & 0 \\
$D$ III & -1 & 1 & 1 \\
$A$ II & -1 & 0 & 0 \\
$C$ II & -1 & -1 & 1 \\
$C$ & 0 & -1 & 0 \\
$C$ I & 1 & -1 & 1 \\
$A$ I & 1 & 0 & 0 \\
$B D \mathrm{I}$ & 1 & 1 & 1 \\
\hline
\end{tabular}

According to the SRFL scheme, a Hamiltonian is called $\Theta$-symmetric, $\Xi$-symmetric, or $\Pi$-symmetric, if respectively,

$$
\Theta H \Theta^{*}=H, \quad \Xi H \Xi^{*}=-H, \quad \Pi H \Pi^{*}=-H .
$$

All possible combinations of the presence or absence of these symmetries are summarised in (Table 4). Here, an entry 0 means that the corresponding symmetry is absent, whereas a non-zero entry \pm 1 indicates that the symmetry is present; in this case, the entry equals the square of the corresponding symmetry operator.

3.2.1. Class $D$ The only restriction here is that a flat-band Hamiltonian $H$ anti-commutes with the real structure $\gamma$ on $W$. Hence, if we set $\Xi:=\gamma$, then $\Xi^{2}=1$ and any $\Xi$ symmetric Hamiltonian gives rise to an IQPV of class $D$.

3.2.2. Class DIII Here, a time-reversal symmetry $T$ on $V, T^{2}=-1$, is present, and extended to $W$ as usual. We may set $\Xi:=\gamma, \Theta:=T$, and $\Pi:=i \Theta \Xi$. Then $\Theta^{2}=-1$, $\Xi^{2}=1$, and $\Pi^{2}=1$, and any $H$ that is symmetric for $\Theta, \Xi$, and $\Pi$ gives rise to an IQPV of class $D$ III.

3.2.3. Class $A$ II In this class we have the symmetries $T$ and $i Q$. As explained in 3.1.6, a IQPV of class $A$ II is the same as an operator $x$ on $V, x^{2}=1$, which commutes with $T$. Hence, if we set $\Theta:=T$, then $\Theta^{2}=-1$ and any $\Theta$-symmetric flat-band Hamiltonian $H$ determines an IQPV of class $A$ II via $x:=H$.

3.2.4. Class $C$ II In addition to the previous symmetries, the particle-hole symmetry $C$ is present and given by $C=\gamma S$ where $S$ is linear, $S^{2}=1$, and $[S, T]=0$. As explained in 3.1.7, an IQPV of class $C$ II is the same as an operator $x, x^{2}=1$, on $V$, such that $T x=x T$ and $S x=-x S$. We may set $\Theta:=T, \Pi:=S, \Xi:=\Theta \Pi$. Then $\Theta^{2}=-1$, $\Pi^{2}=1, \Xi^{2}=-1$, and any flat-band Hamiltonian $H$ symmetric for $\Theta, \Xi$, and $\Pi$ determines an IQPV of class $C$ II via $x:=H$.

3.2.5. Classes $s \geqslant 4$ For $s \geqslant 4$, the generators $S_{1}, S_{2}, S_{3}$ of spin rotation induce an isomorphism $W \cong W^{\sim} \otimes \mathbb{C}^{2}$ and a splitting $\mathbb{A}=A^{W^{\sim}} \otimes \mathbb{H}_{\mathbb{C}}$, as explained in 3.1.8. In this splitting, $\gamma$ corresponds to $T \otimes \mathfrak{c}$ where $T$ is a quaternionic structure on $W^{\sim}$ and $\mathfrak{c}$ the standard quaternionic structure on $\mathbb{C}^{2}$. 
As we have seen, IQPV of class $s \geqslant 4$ correspond to operators $j \in A^{W^{\sim}}$ commuting with $T$ such that $j^{2}=-1$ and $j$ anti-commutes with the first $s-4$ of the operators $j_{T}$, $j_{Q}$, and $j_{C}$, corresponding to $J_{T}, J_{Q}$, and $J_{C}$, respectively. We may proceed as above, with $T$ playing the role of $\gamma$, to establish the correspondence in all real symmetry classes.

3.2.6. Complex class $A$ The complex classes are those where both $J$ and the group of symmetries commute with $Q$. As we have noted, this implies that $J$ and any other symmetries present leave $\mathscr{V}$ invariant and are determined by their restriction to this space. In class $A$, there are no further symmetries present, so the Hamiltonians $H$ on $\mathscr{V}$ determine IQPV in the complex symmetry class $A$.

3.2.7. Complex class AIII In complex class $A$ III, we have the additional symmetry $C=$ $\gamma S$. IQPV in this class correspond to operators on $\mathscr{V}$ anti-commuting with $S$. If we set $\Pi:=S$, then $\Pi^{2}=1$, and $\Pi$-symmetric Hamiltonians determine IQPV in the complex symmetry class $A$ III.

\subsection{From quasi-particle vacua to bulk invariants.}

3.3.1. Van Daele's picture of $K$-theory There exists a plethora of different pictures for $K$-theory, so there is a choice to be made in the construction of $K$-theory classes attached to free-fermion topological phases. We follow the guiding principle to stay as close as possible to the physical model. This leads to different pictures for $K$-theory in the bulk and at the boundary. At the boundary, Kasparov's Fredholm picture is the most natural option, as we shall see in Sect. 4.

In the bulk, the most natural choice is the picture due to Van Daele [52,53], as was first observed by Kellendonk [30]. This picture is pleasantly simple, and it works for graded Banach algebras over the real and complex fields. Since Van Daele's picture appears to be little known, we give a brief exposition for the case of (real) $\mathrm{C}^{*}$-algebras.

Definition 14 (Van Daele's picture for real $K$-theory). Let $A$ be a unital graded real $\mathrm{C}^{*}$-algebra. An odd real Hermitian unitary in $A$ will be called an $O R H U$. Let $\mathscr{F}(A)$ be the set of ORHU in $A$ and $F(A):=\pi_{0}(\mathscr{F}(A))$ the set of path-connected components of $\mathscr{F}(A)$ for the topology induced by the norm. We say that two elements of $\mathscr{F}(A)$ are homotopic if they define the same element of $F(A)$.

For any integer $n \geqslant 1$, we consider the entry-wise grading on $M_{n}(A)=A \otimes M_{n}(\mathbb{C})$. We let $\mathscr{F}_{n}(A):=\mathscr{F}\left(M_{n}(A)\right)$ and $F_{n}(A):=F\left(M_{n}(A)\right)$ where $M_{n}(A)$ has entry-wise grading. For $x \in \mathscr{F}_{m}(A)$ and $y \in \mathscr{F}_{n}(A)$, we define

$$
x \oplus y:=\left(\begin{array}{ll}
x & 0 \\
0 & y
\end{array}\right) \in \mathscr{F}_{m+n}(A)
$$

and $[x] \oplus[y]:=[x \oplus y] \in F_{m+n}(A)$.

Suppose that $\mathscr{F}(A) \neq \varnothing$ and choose $e \in \mathscr{F}(A)$. Then $F_{n}(A)$ becomes an inductive system of sets with the connecting maps $F_{n}(A) \longrightarrow F_{n+1}(A):[x] \longmapsto[x] \oplus[e]$. Consider the inductive limit

$$
F_{\infty}(A):=\lim _{\longrightarrow n} F_{n}(A)
$$


Then $F_{\infty}(A)$ is an Abelian monoid with the addition induced by $\oplus$ and the unit $[e][52$, Proposition 2.7]. We define Van Daele's K R-group

$$
D K R(A):=D K R_{e}(A):=G\left(F_{\infty}(A)\right) .
$$

Here, $G(-)$ denotes the Grothendieck group of an Abelian monoid. In case $A$ is a unital graded $\mathrm{C}^{*}$-algebra, we define $\mathscr{F}_{n}(A), F_{n}(A)$ and $\mathscr{F}_{\infty}(A)$ by dropping all the reality constraints, and we set $D K(A):=G\left(F_{\infty}(A)\right)$.

Remark 13. Note that up to canonical isomorphism, $D K R(A)$ is independent of $e$. Van Daele [52, Definition 2.1] does not impose the condition that the elements defining $\mathscr{F}(A)$ be Hermitian; however, this can be assumed by [52, Proposition 2.5]. If $e$ and $-e$ are homotopic, then $F_{\infty}(A)$ is an Abelian group and the canonical map $F_{\infty}(A) \longrightarrow D K R(A)$ is an isomorphism [52, Proposition 2.11]. The picture of $D K R(A)$ we introduce is due to Roe [47]; Van Daele's original definition of $D K R(A)$ coincides with it by [52, Proposition 3.3]. The independence of $D K R_{e}(A)$ of $e$ [52, Proposition 2.12] can be used to show that $D K R(A)$ is functorial for unital (real) $*$-morphisms. The definition of $D K R(A)$ can be extended to the cases where $\mathscr{F}(A)=\varnothing$ and where $A$ is non-unital [52, Section 3].

Lemma 3. Let $A$ be a unital graded real $C^{*}$-algebra and $e \in \mathscr{F}(A)$ an ORHU. Let $m \geqslant 1$ be an integer. Then the maps

$$
\mathscr{F}_{n}(A) \longrightarrow \mathscr{F}_{n}\left(M_{m}(A)\right): x \longmapsto E_{11} \otimes x \oplus\left(1-E_{11}\right) \otimes e_{n},
$$

where $E_{i j}, 1 \leqslant i, j \leqslant m$, are the standard matrix units and $e_{k}=e \oplus \cdots \oplus e$ is the sum of $k$ copies of e, induce an isomorphism of Abelian groups $D K R_{e}(A) \longrightarrow D K R_{e_{m}}\left(M_{m}(A)\right)$.

For us, the importance of Van Daele's group $D K R(A)$ lies in its relation to (real) $K$-theory, as detailed in the following theorem.

Theorem 2 (Van Daele's picture of real K-theory). Let A be a unital graded real $C^{*}$ algebra resp. a unital graded $C^{*}$-algebra. Then

$$
D K R\left(A \widehat{\otimes} C \ell_{r, s}\right) \cong K R_{s-r+1}(A), \quad \text { resp. } D K\left(A \widehat{\otimes} \mathbb{C} \ell_{r, s}\right) \cong K_{s-r+1}(A) .
$$

Theorem 2 implies that up to isomorphism, $D K R\left(A \widehat{\otimes} C \ell_{r, s}\right)$ depends only on $s-r$ $(\bmod 8)$ and $D K\left(A \widehat{\otimes} \mathbb{C} \ell_{r, s}\right)$ depends only on $s-r(\bmod 2)$. The theorem is proved in Ref. [47, Section 2]. We give a sketch, recalling Roe's construction, as we shall later refer to it. We will make use of Kasparov's bivariant real $K K R$-theory; see [10,27,49].

Proof of Theorem 2, sketch. Applying (1, 1)-periodicity of $D K R$ [47, Lemma 2.4], we may assume that $r \geqslant 1$. Possibly replacing $A$ by a matrix algebra, fix a reference ORHU $e \in \mathscr{F}\left(Q^{s}(A) \widehat{\otimes} C \ell_{r-1, s}\right)$, where $Q^{s}(A):=\mathrm{M}^{S}(A) /(A \widehat{\otimes} \mathbb{K})$ and $\mathbb{K}$ denotes the algebra of compact operators on a separable graded real Hilbert space.

It is well-known that $K R_{s-r+1}(A)=K K R\left(\mathbb{C}, A \widehat{\otimes} C \ell_{r-1, s}\right)$, see [49, Theorem 2.3.8 and remarks following Corollary 2.5.2]. It also standard [49, Proposition 2.3.5] that any class in the latter group can be represented by a cycle of the form $\left(\mathscr{H}_{A} \widehat{\otimes} C \ell_{r-1, s}, 1_{\mathbb{C}}, x\right)$, where $\mathscr{H}_{A}$ is the standard graded real Hilbert $A$-module and $x \in \mathrm{M}^{s}(A) \widehat{\otimes} C \ell_{r-1, s}$ maps to an ORHU in $Q^{s}(A) \widehat{\otimes} C \ell_{r-1, s}$ via the canonical map $\pi: \mathbf{M}^{s}(A) \longrightarrow Q^{s}(A)$.

Let $\partial_{Q}$ be the connecting map for $D K R$, defined in [53], for the short exact sequence

$$
0 \longrightarrow A \widehat{\otimes} \mathbb{K} \longrightarrow \mathrm{M}^{s}(A) \longrightarrow Q^{s}(A) \longrightarrow 0
$$


Because $\mathrm{M}^{s}(A)$ is $K R$-trivial, it is an isomorphism

$$
\partial_{Q}: D K R_{e}\left(Q^{s}(A) \widehat{\otimes} C \ell_{r-1, s}\right) \longrightarrow D K R\left(A \widehat{\otimes} C \ell_{r, s}\right) .
$$

The desired isomorphism

$$
\alpha: K K R\left(\mathbb{C}, A \widehat{\otimes} C \ell_{r-1, s}\right) \longrightarrow D K R\left(A \widehat{\otimes} C \ell_{r, s}\right)
$$

is defined by mapping the $K K R$ class corresponding to $x$ to $\partial_{Q}([x]-[e])$.

3.3.2. Bulk invariants attached to IQPV We will now attach classes in the $K R$-theory of $\mathbb{A}$ to any disordered IQPV with symmetries. The construction is purely algebraic and in terms of Van Daele's picture of (real) $K$-theory, and does not depend on the real $\mathrm{C}^{*}$-algebra $\mathbb{A}$. In fact, we will show that $K R$-theory can be described entirely in terms of the algebraic relations of a disordered IQPV with pseudo-symmetries.

In what follows, let $B$ be an (ungraded) unital real $C^{*}$-algebra. Suppose $r, s \geqslant 0$ and that $\left(K_{1}, \ldots, K_{r}, J_{1}, \ldots, J_{s}\right)$ represent a unital real $*$-morphism $\phi: C \ell_{r, s} \longrightarrow B$.

Definition 15. Define commuting projections $Q_{a}, P_{\alpha} \in B \otimes C \ell_{r, s+1}$ by

$$
\begin{aligned}
& 2 Q_{a}-1=(-1)^{s} K_{a} \otimes k_{a} j_{1}, \quad 2 P_{\alpha}-1=J_{\alpha} \otimes j_{1} j_{\alpha+1} \\
& \quad(a=1, \ldots, r, \alpha=1, \ldots, s) .
\end{aligned}
$$

The product over all of these defines a further projection

$$
P^{r, s}:=Q_{1} \cdots Q_{r} P_{1} \cdots P_{s}
$$

Let $\mathscr{F F}^{r, s}(B)$ be the set of all $J \in B$ satisfying the algebraic relations of an IQPV of symmetry index $(r, s)$, that is

$$
\begin{aligned}
\bar{J}=J=-J^{*}, \quad J^{2}=-1, \\
\\
\quad J K_{a}+K_{a} J=J J_{\alpha}+J_{\alpha} J=0 \quad(a=1, \ldots, r, \alpha=1, \ldots, s) .
\end{aligned}
$$

In case $B$ is not assumed to be real, we make the same definition, dropping the reality constraints on the $K_{a}, J_{\alpha}$ and on $J$.

Proposition 7. The assignment

$$
J \longmapsto\left(J \otimes j_{1}\right) P^{r, s}
$$

defines a bijective map

$$
\mathscr{F}_{F}^{r, s}(B) \longrightarrow \mathscr{F}\left(P^{r, s}\left(B \otimes C \ell_{r, s+1}\right) P^{r, s}\right) .
$$

If $B$ and the $K_{a}, J_{\alpha}$ are not assumed to be real, one has a corresponding bijection

$$
\mathscr{F}_{F}^{r, s}(B) \longrightarrow \mathscr{F}\left(P^{r, s}\left(B \otimes \mathbb{C} \ell_{r, s+1}\right) P^{r, s}\right)
$$

We only give the details of the proof in the real case. It relies on the following lemma. 
Lemma 4. Let $C$ be a unital graded real $C^{*}$-algebra and $x \in C$ be an ORHU. Consider the projection $q \in C \widehat{\otimes} C \ell_{0,1}$ defined by $2 q-1=x \otimes j_{1}$. (Note that $x \otimes j_{1}$ is Hermitian due to the appearance of the graded tensor product.) Then

$$
\mathscr{F}\left(q\left(C \widehat{\otimes} C \ell_{0,1}\right) q\right)=\{(y \otimes 1) q \mid y \in \mathscr{F}(C), x y+y x=0\} .
$$

Moreover, the ORHU $y \in C$ representing an ORHU $(y \otimes 1) q \in q\left(C \widehat{\otimes} C \ell_{0,1}\right) q$ is unique.

Similarly, let $x \in C$ be an odd real skew-Hermitian unitary and $p \in C \widehat{\otimes} C \ell_{1,0}$ be the projection defined by $2 p-1=x \otimes k_{1}$. Then

$$
\mathscr{F}\left(p\left(C \widehat{\otimes} C \ell_{1,0}\right) p\right)=\{(y \otimes 1) p \mid y \in \mathscr{F}(C), x y+y x=0\},
$$

where once again, the representatives y are uniquely determined.

Proof. We only prove the first statement, the proof of the second one being nearly identical. The odd elements of $C \widehat{\otimes} C \ell_{0,1}$ are exactly those of the form

$$
z=\frac{1}{2}(y \otimes 1)+\frac{1}{2}\left(y^{\prime} \otimes j_{1}\right)
$$

where $y$ is odd and $y^{\prime}$ is even. Then $z$ lies in the corner $q\left(C \widehat{\otimes} C \ell_{0,1}\right) q$ if and only if

$$
\left(x \otimes j_{1}\right) z=z=z\left(x \otimes j_{1}\right),
$$

that is, if and only if $y^{\prime}=-x y=y x$. If this is the case, then $z=(y \otimes 1) q$ and $z$ is Hermitian if and only if $y$ is. Finally, in that case, $z$ is unitary in the corner if and only if

$$
\frac{1}{2}(1 \otimes 1)+\frac{1}{2}\left(x \otimes j_{1}\right)=q=z^{2}=(y \otimes 1)^{2} q=\frac{1}{2}\left(y^{2} \otimes 1\right)+\frac{1}{2}\left(y^{2} x \otimes j_{1}\right),
$$

which in turn is equivalent to $y^{2}=1$. By construction, $y$ is uniquely determined by $z$.

Proof of Proposition 7. It is obvious that the $Q_{a}$ and $P_{\alpha}$ are indeed commuting projections. We will prove the statement by two separate inductions with respect to $r$ and $s$. In case $r=s=0$, we have $P^{0,0}=1$, and there is nothing to prove. Suppose that $r=0, s \geqslant 1$, and that the statement has been proved for $(0, s-1)$ and any unital real $C^{*}$-algebra $B$. Since $1 \otimes j_{s+1}$ commutes with $P^{0, s-1}$, Eq. (11) implies that

$$
P^{0, s}\left(B \otimes C \ell_{0, s+1}\right) P^{0, s} \cong P_{s}\left(B^{\prime} \widehat{\otimes} C \ell_{0,1}\right) P_{s}
$$

where

$$
B^{\prime}:=P^{0, s-1}\left(B \otimes C \ell_{0, s}\right) P^{0, s-1} .
$$

Here, the $C \ell_{0,1}$ factor on the right-hand side of the former equation is generated by $j_{s+1}$, and the $C \ell_{0, s}$ factor on the right-hand side of the latter is generated by $j_{1}, \ldots, j_{s}$.

Under the isomorphism in the former equation, $J_{S} \otimes j_{1} j_{s+1}$ is mapped to $x \otimes j_{s+1}$, where $x:=J_{s} \otimes j_{1}$. Hence, we may apply the first part of Lemma 4 with $C=B^{\prime}$ to the right-hand side of the former equation. We conclude that there is a bijection, defined by the equation $a=b P_{s}$, between the

$$
a \in \mathscr{F}\left(P^{0, s}\left(B \otimes C \ell_{0, s+1}\right) P^{0, s}\right),
$$

and the $b \in \mathscr{F}\left(P^{0, s-1}\left(B \otimes C \ell_{0, s}\right) P^{0, s-1}\right)$ anti-commuting with $J_{s} \otimes j_{1}$. By the inductive assumption, the $b \in \mathscr{F}\left(P^{0, s-1}\left(B \otimes C \ell_{0, s}\right) P^{0, s-1}\right)$ are in bijection via 
$b=\left(J \otimes j_{1}\right) P^{0, s-1}$ with the real Hermitian unitaries $J \in B$ anti-commuting with $J_{1}, \ldots, J_{s-1}$. The $b$ anti-commuting with $J_{s} \otimes j_{1}$ are in bijection with the $J$ anticommuting with $J_{1}, \ldots, J_{s}$.

This proves the statement for $(0, s)$. If now $r \geqslant 1$, then similarly

$$
P^{r, s}\left(B \otimes C \ell_{r, s+1}\right) P^{r, s} \cong Q_{r}\left(B^{\prime \prime} \widehat{\otimes} C \ell_{1,0}\right) Q_{r}
$$

where

$$
B^{\prime \prime}:=P^{r-1, s}\left(B \otimes C \ell_{r-1, s+1}\right) P^{r-1, s} .
$$

Here, the $C \ell_{1,0}$ factor on the right-hand side of the former equation is generated by $k_{r}$, whereas the $C \ell_{r-1, s+1}$ factor in the latter is generated by $k_{1}, \ldots, k_{r-1}, j_{1}, \ldots, j_{s+1}$. The assertion now follows by induction on $r$, applying the second part of Lemma 4 to $C=B^{\prime \prime}$ and $x=(-1)^{s} K_{r} \otimes j_{1}$.

In order to see that the ORHU defined by Proposition 7 give classes in the correct $K$-theory, we need the following lemma.

Lemma 5. As before, assume given a unital real $*$-morphism $\phi: C \ell_{r, s} \longrightarrow B$. In addition, suppose there is some $J_{\mathrm{ref}} \in \mathscr{F F}^{r, s}(B)$. Then the graded real $C^{*}$-algebra $B \otimes C \ell_{r, s+1}$ is isomorphic to the graded real $C^{*}$-algebra of $2^{r+s} \times 2^{r+s}$ matrices over $P^{r, s}\left(B \otimes C \ell_{r, s+1}\right) P^{r, s}$ with entry-wise grading.

Proof. We will make a specific choice of isomorphism that will allow us to compute its effect on $K$-theory. We shall index the rows and columns of $2^{r+s} \times 2^{r+s}$ matrices by indices $\varepsilon$ running over $\{ \pm\}^{r+s}$. Set $Q_{\alpha}^{+}:=Q_{\alpha}$ and $Q_{\alpha}^{-}:=1-Q_{\alpha}$, and similarly for $P^{a}$. This enables us to define

$$
P_{\varepsilon}^{r, s}:=Q_{1}^{\varepsilon_{1}} \cdots Q_{r}^{\varepsilon_{r}} P_{1}^{\varepsilon_{r+1}} \cdots P_{s}^{\varepsilon_{r+s}} .
$$

The projections $P_{\varepsilon}^{r, s}$ satisfy

$$
P_{\varepsilon}^{r, s} P_{\varepsilon^{\prime}}^{r, s}=\delta_{\varepsilon \varepsilon^{\prime}} P_{\varepsilon}^{r, s}, \quad \sum_{\varepsilon \in\{ \pm\}^{r+s}} P_{\varepsilon}^{r, s}=1 .
$$

The projections $P_{\varepsilon}^{r, s}$ are all unitarily equivalent. We make a careful choice of unitaries implementing the equivalence. Observe first that

$$
\left.\begin{array}{rl}
\operatorname{Ad}\left(K_{a} J_{\text {ref }} \otimes 1\right)\left(P^{r, s}\right) & =P_{\varepsilon-2 e_{a}}^{r, s} \\
\operatorname{Ad}\left(J_{\alpha} J_{\text {ref }} \otimes 1\right)\left(P^{r, s}\right) & =P_{\varepsilon-2 e_{r+\alpha}}^{r, s}
\end{array}\right\} \quad(a=1, \ldots, r, \alpha=1, \ldots, s) .
$$

Hence, setting

$$
u_{\varepsilon}:=\prod_{1 \leqslant a \leqslant r, \varepsilon_{a}=-}\left(K_{a} J_{\mathrm{ref}} \otimes 1\right) \prod_{1 \leqslant \alpha \leqslant s, \varepsilon_{r+\alpha}=-}\left(J_{\alpha} J_{\mathrm{ref}} \otimes 1\right),
$$

we obtain

$$
u_{\varepsilon} P^{r, s}=P_{\varepsilon}^{r, s} u_{\varepsilon}, \quad u_{\varepsilon}\left(J_{\text {ref }} \otimes j_{1}\right)=\varepsilon\left(J_{\text {ref }} \otimes j_{1}\right) u_{\varepsilon}
$$

where we follow the convention $\varepsilon \equiv \operatorname{sgn} \varepsilon$. Define a map

$$
\Psi: B \otimes C \ell_{r, s+1} \longrightarrow M_{2^{r+s}}(\mathbb{C}) \otimes P^{r, s}\left(B \otimes C \ell_{r, s+1}\right) P^{r, s}
$$


by

$$
\Psi(x):=\sum_{\varepsilon, \varepsilon^{\prime} \in\{ \pm\}^{r+s}} E_{\varepsilon \varepsilon^{\prime}} \otimes P^{r, s} u_{\varepsilon}^{*} x u_{\varepsilon^{\prime}} P^{r, s}=\sum_{\varepsilon, \varepsilon^{\prime} \in\{ \pm\}^{r+s}} E_{\varepsilon \varepsilon^{\prime}} \otimes u_{\varepsilon}^{*} P_{\varepsilon}^{r, s} x P_{\varepsilon^{\prime}}^{r, s} u_{\varepsilon^{\prime}} .
$$

Here, $E_{\varepsilon \varepsilon^{\prime}}$ are the standard matrix units. It is clear that $\Psi$ commutes with $*$ and ${ }^{-}$. A computation using Equation (14) shows that $\Psi$ is in fact a real $*$-morphism. Since the matrix units form a basis of $M_{2^{r+s}}(\mathbb{C})$, it is easy to see that $\Psi$ is bijective.

Remark 14. The result of Lemma 5 is not surprising, as by [44, Example 3.6 and Proposition 3.28], the corner $P^{r, s}\left(B \otimes C \ell_{r, s+1}\right) P^{r, s}$ is Morita equivalent to the closed two-sided ideal generated by $P^{r, s}$. One can show that this ideal is already all of $B \otimes C \ell_{r, s+1}$. Since $B$ is unital by hypothesis, the Brown-Green-Rieffel Theorem [44, Theorem 5.55] applies, and the Morita equivalence implies stable isomorphism.

However, the more precise statement of the lemma will be useful in order to express the bulk classes that we will presently construct in an explicit form.

Circumstantial evidence suggests that Lemma 5 holds in the absence of the assumption that there is an element $J_{\text {ref }}$, although our proof does not work in this case. Indeed, if $\phi$ is injective, then the minimal choice for $B$ is $B=C \ell_{r, s}$. For $r=0, B=C \ell_{0, s}$, one has

$$
B \otimes C \ell_{0, s+1} \cong M_{2^{s}}(E), \quad E_{\mathbb{R}}= \begin{cases}\mathbb{C}, & \text { if } s \equiv 0,1(4), \\ \mathbb{R} \oplus \mathbb{R}, & \text { if } s \equiv 2,3(4) .\end{cases}
$$

For the cases of $s-r \equiv 3$ (4), $\phi$ may fail to be injective. (Otherwise, $C \ell_{r, s}$ is simple.) Setting $r=0$, we have $C \ell_{0, s}=C \ell_{0, s-1} \oplus C \ell_{0, s-1}$, so the minimal choice for $B$ (without the assumption that $\phi$ be injective) is $C \ell_{0, s-1}$, which is $\mathbb{H}_{\mathbb{C}}$ for $s=3$ and $M_{8}(\mathbb{C})$ for $s=7$. In both cases, one has $B \otimes C \ell_{0, s+1} \cong M_{2^{s}}(\mathbb{C})$, and $\mathbb{C}$ is a corner of the tensor product.

Definition 16 (Bulk class of a disordered IQPV with symmetries). We assume that $\mathscr{F} \mathscr{F} r, s(B) \neq \varnothing$ and select $J_{\text {ref }} \in \mathscr{F}_{\mathscr{F}}^{r, s}(B)$. Let $e^{r, s}:=\left(J_{\text {ref }} \otimes j_{1}\right) P^{r, s}$ be the ORHU corresponding to $J_{\text {ref }}$ via Proposition 7. To any $J \in \mathscr{F F F}^{r, s}(B)$, we assign the class

$$
[(J ; \phi)]:=\left[\left(J \otimes j_{1}\right) P^{r, s}\right]-\left[e^{r, s}\right] \in D K R_{e^{r, s}}\left(P^{r, s}\left(B \otimes C \ell_{r, s+1}\right) P^{r, s}\right) .
$$

By Lemmas 5, 3, and Theorem 2, we may consider this as a class in

$$
D K R\left(B \otimes C \ell_{r, s+1}\right)=K R_{s-r+2}(B) .
$$

In case $B=\mathbb{A}$ or $B=\mathrm{A}$ and the $K_{a}, J_{\alpha}$ are operators on $W$ resp. on $V$, we say that $[(J ; \phi)]=\left[\left(J ; K_{1}, \ldots, K_{r}, J_{1}, \ldots, J_{r}\right)\right]$ is the bulk class associated with the disordered IQPV $(J ; \phi)$ of symmetry index $(r, s)$.

By inspecting the proof of Lemma 5, we may determine the class in $D K R(B \otimes$ $\left.C \ell_{r, s+1}\right)$ corresponding to $[(J ; \phi)]$ explicitly.

Lemma 6. Suppose there is some $J_{\text {ref }} \in \mathscr{F F F}^{r, s}(B)$. Then the class in $D K R_{J_{\mathrm{ref}} \otimes j_{1}}(B \otimes$ $\left.C \ell_{r, s+1}\right)$ corresponding to $[(J ; \phi)]$ under the isomorphisms from Lemmas 3 and 5 is

$$
\left[\left(J \otimes j_{1}\right) P^{r, s}+\left(J_{\text {ref }} \otimes j_{1}\right)\left(1-P^{r, s}\right)\right]-\left[J_{\text {ref }} \otimes j_{1}\right] .
$$


Proof. Unless $r+s>0$, there is nothing to prove. Set $n:=2^{r+s-1}$. We abbreviate $e_{0}:=e^{r, s}, p:=P^{r, s}$, and $C:=B \otimes C \ell_{r, s+1}$. Then $e:=e_{0} \oplus-e_{0}$ is homotopic to $-e_{0} \oplus e_{0}$ in $\mathscr{F}_{2}(p C p)$, by [52, p. 191], and hence, $F_{\infty}\left(M_{2}(p C p)\right)$ is an Abelian group isomorphic

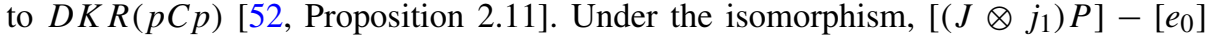
corresponds to the class $[x]$ of the element $x:=\left(J \otimes j_{1}\right) P \oplus\left(-e_{0}\right)$.

The map from Lemma 3 sends $x$ to $x \oplus e_{n-1} \in \mathscr{F}_{2 n}(p B p)$. This is the $2 n \times 2 n$ matrix

$$
E_{++} \otimes\left(J \otimes j_{1}\right) p+\sum_{\varepsilon \neq(+, \ldots,+)} \varepsilon E_{\varepsilon \varepsilon} \otimes e_{0},
$$

where $E_{++}$is the matrix unit corresponding to $\varepsilon=(+, \ldots,+)$.

Let $\Psi$ be the isomorphism from Lemma 5. As $J_{\text {ref }} \otimes j_{1}$ commutes with $p=P^{r, s}$ and $\operatorname{Ad}\left(u_{\varepsilon}\right)\left(J_{\text {ref }} \otimes j_{1}\right)=\varepsilon\left(J_{\text {ref }} \otimes j_{1}\right)$, we find

$$
\begin{aligned}
\Psi\left(J_{\text {ref }} \otimes j_{1}\right) & =\sum_{\varepsilon, \varepsilon^{\prime} \in\{ \pm\}^{r+s}} \varepsilon^{\prime} E_{\varepsilon \varepsilon^{\prime}} \otimes\left(J_{\text {ref }} \otimes j_{1}\right) u_{\varepsilon}^{*} P_{\varepsilon}^{r, s} P_{\varepsilon^{\prime}}^{r, s} u_{\varepsilon^{\prime}} \\
& =\sum_{\varepsilon \in\{ \pm\}^{r+s}} \varepsilon E_{\varepsilon \varepsilon} \otimes\left(J_{\text {ref }} \otimes j_{1}\right) P^{r, s} .
\end{aligned}
$$

Thus we see that $\Psi\left(J_{\text {ref }} \otimes j_{1}\right)=e_{n}$. A similar computation shows that

$$
\Psi\left(\left(J \otimes j_{1}\right) p+\left(J_{\text {ref }} \otimes j_{1}\right)(1-p)\right)=x \oplus e_{n-1} .
$$

Therefore, $x \oplus e_{n-1}$ is identified with the desired element in $M_{2 n}(C)$. Because the class $\left[e_{n}\right]=\left[J_{\text {ref }} \otimes j_{1}\right]$ is zero in the Abelian group $F_{\infty}\left(M_{2 n}(C)\right)$, the assertion follows.

The assignment to $J \in \mathscr{F F F}^{r, s}(B)$ of the $D K R$-class of the ORHU $\left(J \otimes j_{1}\right) P^{r, s}$ in fact gives an alternative definition of real $K$-theory. To that end, let

$$
\mathscr{F F}_{n}^{r, s}(B):=\mathscr{F}^{r, s}\left(M_{n}(B)\right)
$$

where the right-hand side is defined in terms of the elements $1_{n} \otimes K_{a}, a=1, \ldots, r$, and $1_{n} \otimes J_{\alpha}, \alpha=1, \ldots, s$. The sets $\mathscr{F}_{n}^{r, s}(B)$ form an inductive system via

$$
\mathscr{F F}_{n}^{r, s}(B) \longrightarrow \mathscr{F}_{n+1}^{r, s}(B): J \longmapsto J \oplus J_{\text {ref }}:=\left(\begin{array}{cc}
J & 0 \\
0 & J_{\text {ref }}
\end{array}\right) .
$$

Denote the set of path-connected components in the norm topology of $\mathscr{F F}_{n}^{r, s}(B)$ by

$$
F F_{n}^{r, s}(B):=\pi_{0}\left(\mathscr{F}_{n}^{r, s}(B)\right)
$$

For the induced maps, the sets $F F_{n}^{r, s}(B)$ form an inductive system of sets.

Theorem 3 (Real K-theory via IQPV). Let B be an ungraded unital real $C^{*}$-algebra. Let $r, s \geqslant 0$ and $\left(K_{1}, \ldots, K_{r}, J_{1}, \ldots, J_{s}\right)$ represent a unital real $*$-morphism $C \ell_{r, s} \longrightarrow B$. Suppose that $\mathscr{F F}^{r, s}(B) \neq \varnothing$ and select $J_{\text {ref }} \in \mathscr{F}^{r}{ }^{r, s}(B)$.

Then the inductive limit

$$
F F_{\infty}^{r, s}(B):=\lim _{\longrightarrow} F F_{n}^{r, s}(B)
$$


acquires the structure of an Abelian monoid with addition induced by the maps

$$
\mathscr{F}_{m}^{r, s}(B) \times \mathscr{F}_{n}^{r, s}(B) \longrightarrow \mathscr{F}_{m+n}^{r, s}(B):\left(J, J^{\prime}\right) \longmapsto J \oplus J^{\prime}=\left(\begin{array}{ll}
J & 0 \\
0 & J^{\prime}
\end{array}\right)
$$

and unit element induced by $J_{\text {ref. }}$ Moreover, $J \mapsto\left(J \otimes j_{1}\right) P^{r, s}$ induces an isomorphism of Abelian groups

$$
G\left(F F_{\infty}^{r, s}(B)\right) \longrightarrow D K R_{e^{r, s}}\left(P^{r, s}\left(B \otimes C \ell_{r, s+1}\right) P^{r, s}\right)=K R_{s-r+2}(B)
$$

where we consider the reference ORHU $e^{r, s}=\left(J_{\mathrm{ref}} \otimes j_{1}\right) P^{r, s}$.

In case $B$ is not assumed real, we have a corresponding isomorphism

$$
G\left(F F_{\infty}^{r, s}(B)\right) \longrightarrow D K_{e^{r, s}}\left(P^{r, s}\left(B \otimes C \ell_{r, s+1}\right) P^{r, s}\right)=K_{s-r+2}(B) .
$$

Proof. Note that

$$
M_{n}\left(P^{r, s}\left(B \otimes C \ell_{r, s+1}\right) P^{r, s}\right)=\left(1_{n} \otimes P^{r, s}\right)\left(M_{n}(B) \otimes C \ell_{r, s+1}\right)\left(1_{n} \otimes P^{r, s}\right) .
$$

We compute for all $J \in \mathscr{F F}_{n}^{r, s}(B)$ that

$$
\left(\left(J \oplus J_{\mathrm{ref}}\right) \otimes j_{1}\right)\left(1_{n+1} \otimes P^{r, s}\right)=\left(J \otimes j_{1}\right)\left(1_{n} \otimes P^{r, s}\right) \oplus e^{r, s} .
$$

Hence, the map from Proposition 7 defines an isomorphism of the inductive systems $\left(\mathscr{F}_{n}^{r, s}(B)\right)_{n \geqslant 1}$ and $\left(\mathscr{F}_{n}\left(P^{r, s}\left(B \otimes C \ell_{r, s+1}\right) P^{r, s}\right)\right)_{n \geqslant 1}$, where the latter is defined by the reference ORHU $e^{r, s}$.

Inspecting the proof of Proposition 7, the map defined there is a homeomorphism with respect to the topologies induced by the norm. Indeed, in Lemma 4, we have

$$
C \widehat{\otimes} C \ell_{0,1}=C \oplus C j_{1}
$$

as normed vector spaces, and the projection onto the first factor, which is continuous, defines the inverse of the first bijection described there. The same holds for $C \ell_{1,0}$ in place of $C \ell_{0,1}$. Thus, the map from Proposition 7 is a homeomorphism.

Hence, the inductive systems $\left(F F_{n}^{r, s}(B)\right)_{n \geqslant 1}$ and $\left(F_{n}\left(P^{r, s}\left(B \otimes C \ell_{r, s+1}\right) P^{r, s}\right)\right)_{n \geqslant 1}$ are isomorphic, so that we obtain a bijection

$$
F F_{\infty}^{r, s}(B) \longrightarrow F_{\infty}\left(P^{r, s}\left(B \otimes C \ell_{r, s+1}\right) P^{r, s}\right)
$$

In order to see that the left-hand side is an Abelian monoid and this bijection is an isomorphism of such objects, we study the effect of the map from Proposition 7 on the $\oplus$ operation from Equation (16). We compute for $J \in \mathscr{F}_{m}^{r, s}(B)$ and $J^{\prime} \in \mathscr{F}_{n}^{r, s}{ }_{n}^{r}(B)$ :

$$
\left(\left(J \oplus J^{\prime}\right) \otimes j_{1}\right)\left(1_{m+n} \otimes P^{r, s}\right)=\left(J \otimes j_{1}\right)\left(1_{m} \otimes P^{r, s}\right) \oplus\left(J^{\prime} \otimes j_{1}\right)\left(1_{n} \otimes P^{r, s}\right) .
$$

Since $F_{\infty}\left(P^{r, s}\left(B \otimes C \ell_{r, s+1}\right) P^{r, s}\right)$ is an Abelian monoid and its Grothendieck group is $D K R_{e^{r, s}}\left(P^{r, s}\left(B \otimes C \ell_{r, s+1}\right) P^{r, s}\right)$, we have proved the claim. 


\section{The View from the Boundary}

In this section, we will construct a framework for the topological classification of boundaries of topological insulators and superconductors in terms of $K R$-theory. At an interface between bulk systems with different topological invariants, the gap closes, thus defining a gapless boundary system. (Indeed, as we shall see in Sect. 6, this is in a mathematically precise sense a necessary condition for topological non-triviality.)

For this reason, the construction of bulk invariants in Van Daele's picture of real $K$ theory, as developed in the previous section, cannot work at the boundary. The solution for this problem is two-fold: Gapless boundary systems need a gap in the bulk in order to be topologically protected, so we will always consider the boundary together with the bulk. This shall be accomplished by introducing a half-space algebra, as first suggested by Kellendonk-Richter-Schulz-Baldes [31,50]. Secondly, we shall apply an alternative picture of real $K$-theory which allows for the closing of the gap at the boundary.

4.1. The sequence connecting bulk and boundary. We introduce a boundary into our system by considering the half-space semilattice

$$
\hat{\Lambda}:=\Lambda_{\partial} \times \mathbb{N}, \quad \Lambda_{\partial}:=\mathbb{Z}^{d-1}, \quad \mathbb{N}=\{0,1,2, \ldots\},
$$

in which the translational symmetry is broken in the $d$ th coordinate direction. The aim of this section is to exhibit a short exact sequence of real $\mathrm{C}^{*}$-algebras connecting bulk and boundary, as follows:

$$
0 \longrightarrow \mathbb{A}_{\partial} \otimes \mathbb{K}\left(\ell^{2}(\mathbb{N})\right) \stackrel{\iota}{\longrightarrow} \widehat{\mathbb{A}} \stackrel{\varrho}{\longrightarrow} \mathbb{A} \longrightarrow 0
$$

Here, $\mathbb{A}_{\partial}$ is defined just as $\mathbb{A}$, with $\Lambda_{\partial}$ replacing $\Lambda$, and $\widehat{\mathbb{A}}$ is the so-called half-space algebra, we which now proceed to construct.

We define Hilbert spaces on $\hat{\Lambda}$ by setting $\widehat{\mathscr{V}}:=\ell^{2}(\hat{\Lambda}) \otimes V$ and

$$
\widehat{\mathscr{W}}:=\widehat{\mathscr{V}} \oplus \widehat{\mathscr{V}}^{*} \cong \ell^{2}(\hat{\Lambda}) \otimes W .
$$

Of basic importance is the orthogonal projection (called the Szegö projection)

$$
q: \ell^{2}(\Lambda) \longrightarrow \ell^{2}(\hat{\Lambda}) \subseteq \ell^{2}(\Lambda)
$$

Here, we identify $\ell^{2}(\hat{\Lambda})$ with the set of all square-summable sequences $\psi \in \ell^{2}(\Lambda)$ which vanish outside $\hat{\Lambda}$. Implicitly tensoring with $\operatorname{id}_{V}$ or $\operatorname{id}_{W}$, we may consider $q$ as an orthogonal projection defined on $\mathscr{V}$ or $\mathscr{W}$.

The translation $u_{i}:=u_{e_{i}}$, where $e_{i}$ is the $i$ th standard basis vector, defined by

$$
\left(u_{x} \psi\right)(y):=\psi(y-x) \quad\left(x, y \in \Lambda, \psi \in \ell^{2}(\Lambda)\right),
$$

commutes with $q$ if $i<d$. For $i=d$, we obtain the partial isometry

$$
\hat{u}_{d}:=q u_{d} q \in \mathscr{L}\left(\ell^{2}(\hat{\Lambda})\right) .
$$

Then $\hat{u}_{d}$ is characterised by the fact that $\hat{u}_{d}^{*} \hat{u}_{d}=1$ and that $1-\hat{u}_{d} \hat{u}_{d}^{*}$ is the projection onto the space $\ell^{2}\left(\Lambda_{\partial}\right)$ of states supported at the boundary. In other words, $\hat{u}_{d}$ is the unilateral shift in the $d$ th coordinate direction. We are now able to give a definition for the half-space algebra. 
Definition 17 (Half-space covariance algebra). Let $\Omega$ be a compact Hausdorff space, equipped with a continuous right action of the group $\Lambda$. Let $U$ be a finite-dimensional Hilbert space and let $\hat{u}_{x}$, for $x \in \hat{\Lambda}$, be defined by

$$
\left(\hat{u}_{x} \psi\right)(y):=\left(\left(q u_{x} q \otimes \operatorname{id}_{U}\right) \psi\right)(y) \quad\left(x, y \in \hat{\Lambda}, \psi \in \ell^{2}(\hat{\Lambda}) \otimes U\right) .
$$

Define $\widehat{A}^{U}$ to be the closed subalgebra of the set $\mathscr{C}\left(\Omega, C_{u}^{*}(\hat{\Lambda}, U)\right)$ of norm continuous maps $\Omega \longrightarrow C_{u}^{*}(\hat{\Lambda}, U)$ generated by those maps $O$ that are covariant in the sense that

$$
O_{\omega \cdot x}=\hat{u}_{x}^{*} O_{\omega} \hat{u}_{x} \quad(x \in \hat{\Lambda}, \omega \in \Omega) .
$$

Again, $\widehat{A}^{U}$ is a $\mathrm{C}^{*}$-algebra with point-wise operations and the sup norm. Whenever $U$ is real or quaternionic, then $\widehat{A}^{U}$ is real. We define

$$
\widehat{\mathbb{A}}:=\widehat{A}^{W}, \quad \widehat{\mathrm{A}}:=\widehat{A}^{V} .
$$

In order to exhibit $\mathbb{A}$ as a quotient of $\widehat{\mathbb{A}}$, we need to prove that the latter has a crossedproduct-like structure. To that end, we pose the following definition.

Definition 18. Let $B:=\mathscr{C}(\Omega) \otimes \operatorname{End}(W)$. Consider a tuple $(\phi, \hat{V})$, where $\phi: B \longrightarrow C$ is a $*$-morphism to a real $C^{*}$-algebra $C$ and $\hat{V}_{x} \in \mathrm{M}(C)$ (the multiplier algebra), $x \in \hat{\Lambda}$, are real elements. We call $(\phi, \hat{V})$ a real covariant pair if

$$
\hat{V}_{x} \hat{V}_{x}^{*}=1, \quad \hat{V}_{x} \hat{V}_{y}=\hat{V}_{x+y}, \quad \phi\left(\alpha_{x}(f)\right) \hat{V}_{x}=\hat{V}_{x} \phi(f) \quad(x, y \in \hat{\Lambda}, f \in B) .
$$

(Recall from Equation (5) that $\alpha_{x}(f)(\omega)=f(\omega \cdot x)$.) For $B=\mathscr{C}(\Omega) \otimes \operatorname{End}(V)$, we define covariant pairs by dropping the reality constraints from the above definitions.

Remark 15. Observe that the equation $\hat{V} \hat{V}^{*}=1$ for the partial isometries $\hat{V}=\hat{V}_{x}$ in the definition of (real) covariant pairs differs from the equation $\hat{u}^{*} \hat{u}=1$ for the unilateral shift $\hat{u}=\hat{u}_{d}$. The rationale here is the unilateral shift is a truncated left translation, whereas the $\hat{V}$ are truncated right translations: Operators that commute with (truncated) left translations are generated by (truncated) right translations.

Proposition 8. Define $\widehat{R}_{x}:=q R_{x} q$ for $x \in \hat{\Lambda}$ (where $R_{x}$ is right translation by $x$, as in the proof of Theorem 1) and

$$
\left(\hat{\pi}(f)_{\omega} \psi\right)(x)=f(\omega \cdot(-x)) \psi(x) \quad(f \in \mathscr{C}(\Omega) \otimes \operatorname{End}(W), x \in \hat{\Lambda}, \psi \in \widehat{\mathscr{W}}) .
$$

Then $(\hat{\pi}, \widehat{R})$ is a real covariant pair, and together with it, the real $C^{*}$-algebra $\widehat{\mathbb{A}}$ is universal for real covariant pairs. That is, for any real covariant pair $(\phi, \hat{V})$, there is a unique real $*$-morphism $\widehat{\mathbb{A}} \longrightarrow C$, denoted by $\phi \rtimes_{\alpha} \hat{V}$, such that

$$
\left(\phi \rtimes_{\alpha} \hat{V}\right)(\hat{\pi}(f))=\phi(f) \quad\left(\phi \rtimes_{\alpha} \hat{V}\right)\left(\widehat{R}_{x}\right)=\hat{V}_{x} \quad(f \in \mathscr{C}(\Omega) \otimes \operatorname{End}(W), x \in \hat{\Lambda}) .
$$

Similarly, the $C^{*}$-algebra $\widehat{\mathrm{A}}$ is universal for covariant pairs. 
Proof. We limit ourselves to the real case. Let the $\mathrm{C}^{*}$-algebra $\tilde{A}$ be defined by the generators $f \in \mathscr{C}(\Omega) \otimes \operatorname{End}(W)$ and $\hat{\tau}_{x}$, for $x \in \hat{\Lambda}$, by the $*$-algebraic relations of $\mathscr{C}(\Omega) \otimes \operatorname{End}(W)$, by the algebraic relations of $\hat{\Lambda}$, and by the relations

$$
\hat{\tau}_{x} \hat{\tau}_{x}^{*}=1, \quad \hat{\tau}_{x} \hat{\tau}_{y}=\hat{\tau}_{x+y}, \quad \alpha_{x}(f) \hat{\tau}_{x}=\hat{\tau}_{x} f \quad(x, y \in \hat{\Lambda}, f \in \mathscr{C}(\Omega) \otimes \operatorname{End}(W)) .
$$

Then $\tilde{A}$ is a real $\mathrm{C}^{*}$-algebra if we require $\mathscr{C}(\Omega) \otimes \operatorname{End}(W)$ to be a real subalgebra and the generators $\hat{\tau}_{x}, x \in \hat{\Lambda}$, to be real. By definition, $\tilde{A}$ is universal for real covariant pairs.

By restriction to $\hat{\Lambda}$ of the real covariant pair $(\pi, R)$ for the action of the group $\Lambda$ on $\mathscr{C}(\Omega) \otimes \operatorname{End}(W)$, we already know that $(\hat{\pi}, \widehat{R})$ is a real covariant pair. Hence, we obtain a unique real $*$-morphism

$$
\hat{\Phi}:=\hat{\pi} \rtimes_{\alpha} \widehat{R}: \tilde{A} \longrightarrow \mathscr{L}\left(L^{2}(\Omega, P) \otimes \widehat{\mathscr{W}}\right),
$$

where $P$ is the probability measure on $\Omega$ from the proof of Theorem 1 .

Let $\tilde{A}_{\partial}$ denote the universal $C^{*}$-algebra generated by the $f \in \mathscr{C}(\Omega) \otimes \operatorname{End}(W)$ and $\tau_{x}$, for $x \in \Lambda_{\partial}$, subject to the $\mathrm{C}^{*}$-algebraic relations of $\mathscr{C}(\Omega) \otimes \operatorname{End}(W)$, the algebraic relations of $\Lambda_{\partial}$, and the relations

$\tau_{x}^{*} \tau_{x}=\tau_{x} \tau_{x}^{*}=1, \quad \tau_{x} \tau_{y}=\tau_{x+y}, \quad \alpha_{x}(f) \tau_{x}=\tau_{x} f \quad\left(x, y \in \Lambda_{\partial}, f \in \mathscr{C}(\Omega) \otimes \operatorname{End}(W)\right)$.

There is a canonical $*$-morphism $\Phi_{\partial}: \tilde{A}_{\partial} \longrightarrow \tilde{A}$, defined by $\tau_{x} \longmapsto \hat{\tau}_{x}$ and $f \longmapsto f$. By Theorem 1, applied to $\Lambda_{\partial}$ instead of $\Lambda, \widehat{\Phi} \circ \Phi_{\partial}$ is injective. Moreover, $\tilde{A}_{\partial}$ is a closed *-subalgebra of $\tilde{A}$.

We now apply Murphy's results on crossed products by ordered groups [41]. By definition, $\tilde{A}$ satisfies the universality for covariant pairs stated in [41, Proposition 1.1] for Murphy's crossed product $\tilde{A}_{\partial} \rtimes \mathbb{N}$, and hence, $\tilde{A}$ is canonically isomorphic to that crossed product. Since $\widehat{\Phi} \circ \Phi_{\partial}$ is faithful, [41, Theorem 4.4] applies, and it follows that the real $*$-morphism $\widehat{\Phi}$ is injective.

It remains to be shown that im $\widehat{\Phi}=\widehat{\mathbb{A}}$. To that end, we refer back to the proof of Theorem 1 . The statement that im $\widehat{\Phi} \subseteq \widehat{\mathbb{A}}$ follows in much the same way as the corresponding statement for $\mathbb{A}$. For the converse inclusion, we begin with some preparations which are largely similar to the corresponding steps in the bulk: Recall the definition of the maps $S_{n}$ and the functions $f_{n}^{i}$; since we may consider $\mathscr{L}(\widehat{\mathscr{W}})$ as a subset of $\mathscr{L}(\mathscr{W})$ by the use of the projection $q$, we may restrict $S_{n}$ to $\mathscr{L}(\widehat{\mathscr{W}})$. For $T \in \mathscr{L}(\widehat{\mathscr{W}})$, we find that

$$
\widehat{S}_{n}(T):=S_{n}(T)=\sum_{i} M_{\hat{f}_{n}^{i}}^{*} T M_{\hat{f}_{n}^{i}}, \quad \hat{f}_{n}^{i}:=\left.f_{n}^{i}\right|_{\hat{\Lambda}} \quad(T \in \mathscr{L}(\widehat{\mathscr{W}})),
$$

where $M_{f}$ is the operator of multiplication by $f$. In particular, $\widehat{S}_{n}$ is a completely positive endomorphism of $\mathscr{L}(\widehat{\mathscr{W}})$. Hence, as for $S_{n}, \widehat{S}_{n}$ is a contraction. We see as before that

$$
\widehat{S}_{n}(T)=\sum_{x, y \in \hat{\Lambda}} \phi_{n}(x-y) T(x, y)|x\rangle\langle y| \quad(T \in \mathscr{L}(\widehat{\mathscr{W}})) .
$$

Then $\widehat{S}_{n}$ leaves controlled operators on $\ell^{2}(\hat{\Lambda})$ invariant, and therefore also the algebra $C_{u}^{*}(\hat{\Lambda}, W)$. Defining, for $T \in \widehat{\mathbb{A}}, \widehat{S}_{n}(T)$ by $\widehat{S}_{n}(T)_{\omega}:=\widehat{S}_{n}\left(T_{\omega}\right)$ for all $\omega \in \Omega$, we obtain a contractive, completely positive endomorphism of $\widehat{\mathbb{A}}$. As previously, it follows that $S_{n}(T)$ converges to $T$ for any $T \in \widehat{\mathbb{A}}$. 
The remaining steps differ from our previous considerations. Let $T: \Omega \longrightarrow$ $C_{u}^{*}(\hat{\Lambda}, W)$ be norm continuous and satisfy the covariance condition in Definition 17 . Setting

$$
x \leqslant y \quad: \Longleftrightarrow y-x \in \hat{\Lambda}
$$

defines a total preorder on $\hat{\Lambda}$. Therefore, we may write $\widehat{S}_{n}(T)=\mathrm{I}+\mathrm{II}$ where

$$
\mathrm{I}_{\omega}=\sum_{x, y \in \hat{\Lambda}, x \leqslant y} \phi_{n}(x-y) T_{\omega}(x, y)|x\rangle\langle y|
$$

and the sum defining II extends over all $x, y \in \hat{\Lambda}, x>y$. For $x \leqslant y$, we have

$$
\langle x| \widehat{R}_{y-x}=\langle y|
$$

so that

$$
\mathrm{I}_{\omega}=\sum_{x, y \in \hat{\Lambda}, x \leqslant y} \phi_{n}(x-y) T_{\omega \cdot x}(0, y-x)|x\rangle\langle y|=\sum_{g \in \hat{\Lambda} \cap \operatorname{supp} \phi_{n}} \pi\left(f_{n, g}^{\mathrm{I}}\right)_{\omega} \widehat{R}_{g}
$$

where

$$
f_{n, g}^{\mathrm{I}}(\omega):=\phi_{n}(g) T_{\omega}(0, g) \quad(n \geqslant 0, g \in \hat{\Lambda}, \omega \in \Omega) .
$$

Similarly, for $x>y$, we have

$$
\left(\widehat{R}_{x-y}\right)^{*} w|y\rangle=w|x\rangle \quad(w \in W)
$$

so that

$$
\mathbb{I}_{\omega}=\sum_{x, y \in \hat{\Lambda}, x>y} \phi_{n}(x-y) T_{\omega \cdot y}(x-y, 0)|x\rangle\langle y|=\sum_{g \in\left(\hat{\Lambda} \backslash \Lambda_{\partial}\right) \cap\left(-\operatorname{supp} \phi_{n}\right)}\left(\widehat{R}_{g}\right)^{*} \pi\left(f_{n, g}^{\mathbb{I I}}\right)_{\omega}
$$

where

$$
f_{n, g}^{\mathbb{I I}}(\omega):=\phi_{n}(-g) T_{\omega}(g, 0) \quad(n \geqslant 0, g \in \hat{\Lambda}, \omega \in \Omega) .
$$

This proves that $\widehat{S}_{n}(T) \in \operatorname{im} \widehat{\Phi}$; since im $\widehat{\Phi}$ is closed, it also contains $T$, proving that $\widehat{\Phi}$ is surjective, and hence, the assertion, as $\tilde{A}$ satisfies the claimed universality.

The following theorem constructs the desired sequence (17).

Theorem 4. There is a unique surjective real $*$-morphism $\varrho: \widehat{\mathbb{A}} \longrightarrow \mathbb{A}$ such that

$$
\varrho(\hat{\pi}(f))=\pi(f), \quad \varrho\left(\widehat{R}_{x}\right)=R_{x} \quad(f \in \mathscr{C}(\Omega) \otimes \operatorname{End}(W), x \in \hat{\Lambda}) .
$$

The kernel of $\varrho$ is the closed ideal (e) generated by the projection $e:=1-\left(\widehat{R}_{d}\right)^{*} \widehat{R}_{d}$, where $R_{d}:=R_{e_{d}}$. It is isomorphic as a real $C^{*}$-algebra to $\mathbb{A}_{\partial} \otimes \mathbb{K}\left(\ell^{2}(\mathbb{N})\right)$. The same holds for $\widehat{\mathrm{A}}$, with $W$ replaced by $V$, and without the reality constraints. 
Proof. As before, we limit our detailed proof to the real case. The unique existence and the surjectivity of $\varrho$ follow directly from Proposition 8 and Theorem 1. It is also clear from the definitions that the projection $e$ is contained in the kernel of $\varrho$.

We abbreviate $\widehat{S}:=\hat{u}_{d}$ and $\hat{e}:=1-\widehat{S} \widehat{S}^{*}$, the orthogonal projection onto $\ell^{2}\left(\Lambda_{\partial}\right)$. By Theorem 1, there is a unique real $*$-morphism $\phi: \mathbb{A} \longrightarrow \mathbb{A} \otimes M_{2}\left(\mathscr{L}\left(\ell^{2}(\mathbb{N})\right)\right)$, such that

$$
\left.\begin{array}{c}
\phi(\pi(f)):=\pi(f) \otimes\left(\begin{array}{ll}
1 & 0 \\
0 & 1
\end{array}\right) \\
\phi\left(R_{(x, n)}\right):=R_{(x, n)} \otimes\left(\begin{array}{cc}
\widehat{S}^{*} & 0 \\
\hat{e} & \widehat{S}
\end{array}\right)^{n}
\end{array}\right\} \quad\left(f \in \mathscr{C}(\Omega) \otimes \operatorname{End}(W), x \in \mathbb{Z}^{d-1}, n \in \mathbb{Z}\right) .
$$

In fact, observe that

$$
\left(\begin{array}{cc}
\widehat{S}^{*} & 0 \\
\hat{e} & \widehat{S}
\end{array}\right)\left(\begin{array}{cc}
\widehat{S} & \hat{e} \\
0 & \widehat{S}^{*}
\end{array}\right)=\left(\begin{array}{ll}
1 & 0 \\
0 & 1
\end{array}\right)=\left(\begin{array}{cc}
\widehat{S} & \hat{e} \\
0 & \widehat{S}^{*}
\end{array}\right)\left(\begin{array}{cc}
\widehat{S}^{*} & 0 \\
\hat{e} & \widehat{S}
\end{array}\right) .
$$

By Proposition 8, we have a unique real $*$-morphism $\hat{\phi}: \widehat{\mathbb{A}} \longrightarrow \mathbb{A} \otimes \mathscr{L}\left(\ell^{2}(\mathbb{N})\right)$,

$$
\left.\begin{array}{l}
\hat{\phi}(\hat{\pi}(f)):=\pi(f) \otimes 1 \\
\hat{\phi}\left(\widehat{R}_{(x, n)}\right):=R_{(x, n)} \otimes\left(\widehat{S}^{*}\right)^{n}
\end{array}\right\} \quad\left(f \in \mathscr{C}(\Omega) \otimes \operatorname{End}(W), x \in \mathbb{Z}^{d-1}, n \in \mathbb{N}\right)
$$

As in the proof of Proposition 8 , using the obvious action of $\mathbb{R} / \mathbb{Z}$ on $\mathscr{L}\left(\ell^{2}(\mathbb{N})\right)$, we see that $\hat{\phi}$ is injective, and hence defines a real $*$-isomorphism onto its image.

By the definition of $\phi$, the image of the upper left component $\phi_{11}$ is contained in the image of $\hat{\phi}$. We may therefore define

$$
\sigma: \mathbb{A} \longrightarrow \widehat{\mathbb{A}}, \quad \sigma:=(\hat{\phi})^{-1} \circ \phi_{11}
$$

This map is not a $*$-morphism, but it is a completely positive contraction intertwining the $*$ operations. Moreover, we have

$$
\phi_{11}(x y)=\phi_{11}(x) \phi_{11}(y)+\phi_{12}(x) \phi_{21}(y) \quad(x, y \in \mathbb{A})
$$

where $\phi_{12}(x) \phi_{21}(y)$ is contained in the ideal generated by $1 \otimes \hat{e}$. By $\hat{\phi}^{-1}$, this ideal is mapped onto the ideal generated by $e$. It follows that $\sigma$ is a section of $\varrho$, as it is sufficient to check this statement on generators. We claim that $x-\sigma(\varrho(x))$ is contained in the closed ideal generated by $e$. By the same argument as in the previous paragraph, it is sufficient to check on generators $x$ that $\hat{\phi}(x)-\phi_{11}(\varrho(x))$ is contained in the closed ideal generated by $1 \otimes \hat{e}$. But this expression actually vanishes on generators.

Now we prove $\operatorname{ker} \varrho \subseteq(e)$. Thus, let $x \in \widehat{\mathbb{A}}, \varrho(x)=0$. Then we have

$$
x=x-\sigma(\varrho(x)) \in(e),
$$

proving that indeed, $\operatorname{ker} \varrho=(e)$.

It remains to prove that the ideal $(e)$ is isomorphic to $\mathbb{A}_{\partial} \otimes \mathbb{K}\left(\ell^{2}(\mathbb{N})\right)$. Define

$$
\phi_{\partial}(a \otimes|m\rangle\langle n|):=\left(R_{d}^{*}\right)^{m} a\left(R_{d}\right)^{n} \otimes|m\rangle\langle n| \in \mathbb{A} \otimes \mathscr{L}\left(\ell^{2}(\mathbb{N})\right) \quad\left(a \in \mathbb{A}_{\partial}\right) .
$$


Then $\phi_{\partial}$ is a real $*$-morphism on $\mathbb{A}_{\partial} \otimes \mathscr{L}_{\text {fin }}\left(\ell^{2}(\mathbb{N})\right)$, where $\mathscr{L}_{\text {fin }}$ denotes the finite rank operators. It is easy to see that $\phi_{\partial}$ is an isometry on $\mathbb{A}_{\partial} \otimes \mathscr{L}_{\text {fin }}\left(\ell^{2}(\mathbb{N})\right)$, and hence it extends to $\mathbb{A}_{\partial} \otimes \mathbb{K}\left(\ell^{2}(\mathbb{N})\right)$ as an isometric real $*$-morphism. Now, observe that

$$
\left(R_{d}^{*}\right)^{m} a\left(R_{d}\right)^{n} \otimes|m\rangle\langle n|=\hat{\phi}\left(\widehat{R}_{e_{d}}^{*}\right)^{m}(a \otimes 1)(1 \otimes \hat{e}) \hat{\phi}\left(\widehat{R}_{e_{d}}\right)^{n} .
$$

Thus, it is clear that the image of $\phi_{\partial}$ equals the closed ideal of im $\hat{\phi}$ generated by $1 \otimes \hat{e}$, proving the claim, and thereby, the theorem.

4.2. Real K-theory: Kasparov's Fredholm picture. In the previous subsection, we have constructed the bulk-to-boundary sequence (17). Our next aim, which we will address in Sect. 4.3, is to attach boundary $K$-theory classes to every disordered IQPV of a given symmetry index. In order to do that, we will need a different picture of real $K$-theory, due to Kasparov [28]. This we introduce in the present section.

Kasparov's picture of $K$-theory is best known in the context of Kasparov's bivariant $K K$-theory, which unifies both $K$-theory and its dual theory, $K$-homology. We have encountered the bivariant theory before, in the proof of Theorem 2 in Sect. 3. It continues to be an important technical tool for us, and we shall employ it in the present section, in the proof of Proposition 9, and in the proof of our main result in Sect. 5. For the purpose of constructing boundary invariants and stating bulk-boundary correspondence, it will be unnecessary to give a the definition of $K K$-theory, and we will eschew it because of its considerable technical difficulty. For more details, see [10,27,28,49].

The following definition of Kasparov's picture of $K$-theory, which does not appeal to the bivariant theory, is taken from [28].

Definition 19 (Kasparov's Fredholm picture of real K-theory). Let $A$ be a trivially graded real $C^{*}$-algebra. Let $\mathbb{K}$ denote the real $C^{*}$-algebra of compact operators on a separable real Hilbert space. Let $\mathrm{M}^{s}(A):=\mathrm{M}(A \otimes \mathbb{K})$ be the stable multiplier algebra.

Suppose we are given a pair $(F, \psi)$ consisting of an ungraded real $*$-morphism $\psi: C \ell_{p, q-1} \longrightarrow \mathrm{M}^{s}(A)$ and a real operator $F \in \mathrm{M}^{S}(A)$ such that all of the expressions

$$
\psi\left(k_{a}\right) F+F \psi\left(k_{a}\right), \quad \psi\left(j_{\alpha}\right) F+F \psi\left(j_{\alpha}\right), \quad F^{*}+F, \quad 1+F^{2},
$$

where $a=1, \ldots, p$ and $\alpha=1, \ldots, q-1$, are contained in $A \otimes \mathbb{K}$. Such a pair is called a Kasparov $K R_{p, q}$ cycle. It is called degenerate if all the above expressions vanish. Let $\mathbb{E}_{p, q}(A)$ be the set of Kasparov $K R_{p, q}$ cycles and $\mathbb{D}_{p, q}(A)$ be the set of degenerate Kasparov $K R_{p, q}$ cycles.

Two cycles $\left(F_{j}, \psi_{j}\right), j=0,1$, are called homotopy equivalent if there is a Kasparov cycle $(F, \psi) \equiv\left(F_{t}, \psi_{t}\right) \in \mathbb{E}_{p, q}(\mathscr{C}([0,1], A))$ such that

$$
\left.(F, \psi)\right|_{t=j}=\left(F_{j}, \psi_{j}\right) \quad(j=0,1) .
$$

They are called unitarily equivalent if there is an even real unitary $u \in M^{S}(A)$ such that

$$
\psi_{0}=u^{*} \psi_{1} u, \quad F_{0}=u^{*} F_{1} u .
$$

The quotient of $\mathbb{E}_{p, q}(A)$ with respect to the equivalence relation generated by homotopy and unitary equivalence is denoted by $\overline{\mathbb{E}}_{p, q}(A)$. It is an Abelian semigroup with respect to the direct sum of cycles. (This operation is well defined, as $M^{S}(A) \oplus M^{S}(A) \cong M^{S}(A)$ by Kasparov's stabilisation theorem.) Let $\overline{\mathbb{D}}_{p, q}(A)$ be the sub-semigroup generated by the image of $\mathbb{D}_{p, q}(A)$ in $\overline{\mathbb{E}}_{p, q}(A)$. 
By definition, Kasparov's $K R_{p, q}$-group is the Abelian monoid

$$
K R_{p, q}(A):=\overline{\mathbb{E}}_{p, q}(A) / \overline{\mathbb{D}}_{p, q}(A) .
$$

It is in fact an Abelian group. By dropping the reality constraints, we may define in the same way the Abelian group $K_{p, q}(A)$, for any trivially graded $\mathrm{C}^{*}$-algebra $A$. This group is called Kasparov's $K_{p, q}$-group.

Proposition 9. Let $A$ be an ungraded real $C^{*}$-algebra resp. an ungraded $C^{*}$-algebra. Then $K R_{p, q}(A) \cong K R_{q-p}(A)$ resp. $K_{p, q}(A) \cong K_{q-p}(A)$.

The proof of the proposition is somewhat technical (although not hard). We defer it to the end of the section. One immediate consequence of the proposition is the following standard simplification, well-known in $K K$-theory.

Lemma 7. Any element of $K R_{p, q}(A)$ or $K_{p, q}(A)$ can be represented by a cycle $(F, \psi)$ for which all of the following expressions vanish:

$\psi\left(k_{a}\right) F+F \psi\left(k_{a}\right), \quad \psi\left(j_{\alpha}\right) F+F \psi\left(j_{\alpha}\right), \quad F^{*}+F \quad(a=1, \ldots, p, \alpha=1, \ldots, q-1)$.

Proof. By Proposition 9 and standard simplifications in $K K$-theory [10, Proposition 17.4.2], we may assume that $F$ is skew-Hermitian. If there is some $a$ such that the anti-commutator $\psi\left(k_{a}\right) F+F \psi\left(k_{a}\right) \neq 0$, we may take $a$ is minimal for this property. Form

$$
\tilde{F}:=\frac{1}{2}\left(1-\operatorname{Ad}\left(\psi\left(k_{a}\right)\right)\right)(F) .
$$

One computes easily that $\tilde{F}$ is skew-Hermitian and $\tilde{F}^{2}+1 \in A \otimes \mathbb{K}$. As for $b<a$, $\operatorname{Ad}\left(\psi\left(k_{b}\right)\right)$ commutes with $\operatorname{Ad}\left(\psi\left(k_{a}\right)\right)$, we see that $\operatorname{Ad}\left(k_{b}\right)(\tilde{F})=-\tilde{F}$ for all $b \leqslant a$. Similarly, $\psi\left(j_{\alpha}\right) F+F \psi\left(j_{\alpha}\right) \in A \otimes \mathbb{K}$. As $\tilde{F}$ is a "compact perturbation" of $F,(\tilde{F}, \psi)$ represents the same class as $(F, \psi)$ [10, Proposition 17.2.5]. Thus, we may assume that $F$ anti-commutes with all the $\psi\left(k_{a}\right)$. Arguing similarly, we may assume also that it anti-commutes with all the $\psi\left(j_{\alpha}\right)$.

In the following sections, we will identify all (real) $K$-groups with their counterparts in Kasparov's picture whenever we see fit to do so. We shall use the notation $K R_{q-p}(A)$ interchangeably to denote $K R_{p, q}(A)$ or any other realisation of $K R$ theory. We end the subsection by a proof of the above proposition.

Proof of Proposition 9. We will sketch the construction leading to the proof of the statement that $K R_{p, q}(A)$ is isomorphic to the Kasparov group $K K R\left(C \ell_{q-1, p}, A \otimes C \ell_{0,1}\right)$, which is well-known to be isomorphic to $K R_{q-p}(A)$. The complex case is proved in completely the same way.

Let $(F, \psi)$ be a Kasparov $K R_{p, q}$ cycle, and set

$$
K_{a}:=\psi\left(k_{a}\right), \quad J_{\alpha}:=\psi\left(j_{\alpha}\right) \quad(a=1, \ldots, p, \alpha=1, \ldots, q-1) .
$$

These operators are ungraded. We introduce a further Clifford algebra $C \ell_{0,1}$ into the picture, equipped now with its natural grading and its odd generator $j_{1}$. This allows us to define an even real $*$-morphism

$$
\phi: C \ell_{q-1, p} \longrightarrow \mathrm{M}^{s}\left(A \otimes C \ell_{0,1}\right)=\mathrm{M}^{s}(A) \otimes C \ell_{0,1}
$$


of graded real $\mathrm{C}^{*}$-algebras, as follows:

$$
\begin{cases}k_{\alpha} \longmapsto J_{\alpha} \otimes j_{1} & \text { for } \alpha=1, \ldots, q-1, \\ j_{a} \longmapsto K_{a} \otimes j_{1} & \text { for } a=1, \ldots, p .\end{cases}
$$

(Note here that the roles of $\alpha$ and $a$ are exchanged on the side of the Clifford generators, as is necessary to perform the desired parity reversal.) Then $G:=F \otimes j_{1} \in \mathrm{M}^{S}\left(A \otimes C \ell_{0,1}\right)$ is real and odd, and all of the expressions

$$
G \phi\left(k_{\alpha}\right)+\phi\left(k_{\alpha}\right) G, \quad G \phi\left(j_{a}\right)+\phi\left(j_{a}\right) G, \quad G^{2}-1, \quad G^{*}-G,
$$

for $a=1, \ldots, p$ and $\alpha=1, \ldots, q-1$, are contained in $A \otimes C \ell_{0,1} \otimes \mathbb{K}$.

Suppose now that $\mathscr{H}$ is a separable real Hilbert space such that $\mathbb{K}=\mathbb{K}(\mathscr{H})$. Then $\mathrm{M}^{s}\left(A \otimes C \ell_{0,1}\right)$ is the graded real $\mathrm{C}^{*}$-algebra of adjointable operators on the standard graded real Hilbert- $C^{*}$-module $\mathscr{E}=\mathscr{H}_{A \otimes C \ell_{0,1}}$ over $A \otimes C \ell_{0,1}$. It follows that $(\mathscr{E}, \phi, G)$ represents a class in $K K R\left(C \ell_{q-1, p}, A \otimes C \ell_{0,1}\right)$. The remainder of the proof is along the lines of that of $[27, \S 6$, Corollary 1 to Theorem 2].

4.3. From quasi-particle vacua to boundary classes. In this section, we assign to any IQPV of any given symmetry index, an element in a suitable $K R$ group of the boundary algebra $\mathbb{A}_{\partial}$. An intermediate step is given by the following definition.

Definition 20 (Quasi-particle vacua with boundary). A disordered IQPV with boundary is a real skew-Hermitian $\widehat{J} \in \widehat{\mathbb{A}}$, i.e.

$$
\widehat{J}^{*}=-\widehat{J}=\widehat{J}^{\top}
$$

such that $J:=\varrho(\widehat{J}) \in \mathbb{A}$ is a disordered IQPV. By Theorem 4, this means that

$$
\widehat{J}^{2} \equiv-1 \quad\left(\bmod \mathbb{A}_{\partial} \otimes \mathbb{K}\left(\ell^{2}(\mathbb{N})\right)\right) .
$$

A tuple $(\widehat{J} ; \phi)=\left(\widehat{J} ; K_{1}, \ldots, K_{r}, J_{1}, \ldots, J_{s}\right)$ is called a disordered IQPV with boundary of symmetry index $(r, s)$ if $\widehat{J}$ is a disordered IQPV with boundary and moreover, the tuple $(J ; \phi)=\left(J ; K_{1}, \ldots, K_{r}, J_{1}, \ldots, J_{s}\right)$ is a disordered IQPV of index $(r, s)$, where $J:=\varrho(\widehat{J})$.

Explicitly, this means the following: $\widehat{J} \in \widehat{\mathbb{A}}$ is a real skew-Hermitian operator; $K_{a}$, $J_{\alpha}$, define a real $*$-morphism $\phi: C \ell_{r, s} \longrightarrow \operatorname{End}(W)$; and the expressions

$$
\widehat{J} K_{a}+K_{a} \widehat{J}, \quad \widehat{J} J_{\alpha}+J_{\alpha} \widehat{J}, \quad \widehat{J}^{2}+1 \quad(a=1, \ldots, r, \alpha=1, \ldots, s)
$$

all belong to $\mathbb{A}_{\partial} \otimes \mathbb{K}\left(\ell^{2}(\mathbb{N})\right)$. Similarly, in the complex case, we define charge-preserving disordered IQPV with boundary resp. disordered IQPV with boundary of complex symmetry index $(r, s)$ by replacing $\widehat{\mathbb{A}}$ by $\widehat{\mathrm{A}}$ and dropping the reality constraints.

Proposition 10. Any disordered IQPV with boundary of (complex) symmetry index $(r, s)$ represents a class in $K R_{s-r+1}\left(\mathbb{A}_{\partial}\right)$ resp. in $K_{s-r+1}\left(\mathrm{~A}_{\partial}\right)$.

Proof. By Proposition 9, a Kasparov $K R_{r, s+1}$ cycle for $\mathbb{A}_{\partial}$ represents a class in the desired group $K R_{s-r+1}\left(\mathbb{A}_{\partial}\right)$. Comparing Definitions 19 and 20, a disordered IQPV with boundary $(\widehat{J} ; \phi)$ of symmetry index $(r, s)$ is distinguished from a Kasparov $K R_{r, s+1}$ cycle only by lying in $\widehat{\mathbb{A}}$ instead of $\mathrm{M}^{s}\left(\mathbb{A}_{\partial}\right)$. 
Thus, it suffices to exhibit $\widehat{\mathbb{A}}$ inside of $\mathrm{M}^{s}\left(\mathbb{A}_{\partial}\right)=\mathrm{M}\left(\mathbb{A}_{\partial} \otimes \mathbb{K}\left(\ell^{2}(\mathbb{N})\right)\right)$. To that end, recall from Theorem 4 and its proof that $\widehat{\mathbb{A}}$ is isomorphic to the closed $*$-subalgebra $B$ of $\mathbb{A} \otimes \mathscr{L}\left(\ell^{2}(\mathbb{N})\right)$ generated by

$$
a \otimes 1, \quad R_{d} \otimes \widehat{S}^{*} \quad\left(a \in \mathbb{A}_{\partial}\right) .
$$

Moreover, $\mathbb{A}_{\partial} \otimes \mathbb{K}\left(\ell^{2}(\mathbb{N})\right)$ is isomorphic to the closed ideal $I \subseteq B$ generated by $1 \otimes \hat{e}$, where $\hat{e}=1-\widehat{S} \widehat{S}^{*}$. By the definition of the multiplier algebra, there is a unique $*-$ morphism $B \longrightarrow \mathrm{M}^{S}\left(\mathbb{A}_{\partial}\right)$ that is the identity on $I$ [15, Proposition 3.7 (i)]. As $B$ is unital, it is surjective. To see that the $*$-morphism $\varrho$ is injective, it will be sufficient to prove that $I$ is essential in $B$ [15, Proposition 3.7 (ii)]. Let $b \in B$ such that $b I=0$. Then

$b(\psi \otimes|n\rangle)=b\left(R_{d}^{*} \otimes \widehat{S}\right)^{n}(1 \otimes \hat{e})\left(\left(R_{d}\right)^{n} \psi \otimes|0\rangle\right)=0 \quad\left(\psi \in L^{2}(\Omega, P) \otimes \mathscr{W}, n \in \mathbb{N}\right)$.

Since $\psi$ and $n$ were arbitrary and $B$ is represented faithfully on $L^{2}(\Omega, P) \otimes \mathscr{W} \otimes \ell^{2}(\mathbb{N})$, we conclude that $b=0$, so that $I$ is essential.

By definition, a disordered IQPV with boundary $\left(\widehat{J} ; K_{1}, \ldots, K_{r}, J_{1}, \ldots, J_{S}\right)$ of symmetry index $(r, s)$ determines a disordered bulk IQPV

$$
J=\rho(\widehat{J}) \in \mathbb{A}
$$

of the same symmetry index $(r, s)$. Since $\varrho$ is surjective, any bulk IQPV is thus determined. However, we can do better and always give a canonical representative. Indeed, let $(J ; \phi)=\left(J ; K_{1}, \ldots, K_{r}, J_{1}, \ldots, J_{s}\right)$ be a disordered IQPV with of symmetry index $(r, s)$. We may thus define

$$
\widehat{J}_{\omega}:=q J_{\omega} q \quad(\omega \in \Omega) .
$$

Then $(\widehat{J} ; \phi)=\left(\widehat{J} ; K_{1}, \ldots, K_{r}, J_{1}, \ldots, J_{s}\right)$ is a disordered IQPV with boundary of symmetry index $(r, s)$, as follows immediately from the following lemma. The same construction also works in the case of a disordered IQPV of complex symmetry index $(r, s)$.

Lemma 8. Let $a \in \mathbb{A}$. Then $\rho(q a q)=a$.

Proof. Recall the covariant pairs $(\pi, R)$ and $(\widehat{\pi}, \widehat{R})$ from Theorem 1 and Proposition 8 , together with their proofs. Let $f \in \mathscr{C}(\Omega) \otimes \operatorname{End}(W)$ and $x \in \hat{\Lambda}$. Then

$$
q \pi(f) R_{x} q=q \pi(f) q R_{x} q=\widehat{\pi}(f) \widehat{R}_{x},
$$

as $\pi(f)$ commutes with $q$. It follows from Theorem 4 that

$$
\rho\left(q \pi(f) R_{x} q\right)=\rho\left(\widehat{\pi}(f) \widehat{R}_{x}\right)=\rho(\widehat{\pi}(f)) \rho\left(\widehat{R}_{x}\right)=\pi(f) R_{x} .
$$

Similarly,

$$
q \pi(f) R_{-x} q=\widehat{\pi}(f) \widehat{R}_{x}^{*},
$$

which implies that

$$
\rho\left(q \pi(f) R_{-x} q\right)=\pi(f) R_{x}^{*}=\pi(f) R_{-x} .
$$

The operators $\pi(f) R_{x}$, for $f \in \mathscr{C}(\Omega) \otimes \operatorname{End}(W)$ and $x \in \Lambda$, span a dense subalgebra of $\mathbb{A}$, in view of Theorem 1 . Since $\hat{\Lambda} \cup(-\hat{\Lambda})=\Lambda$, the assertion follows from the linearity and continuity of $\rho$. 
Definition 21 (Boundary class of an IQPV). Let $\phi: C \ell_{r, s} \longrightarrow \operatorname{End}(W)$ be a real *morphism (resp. $\phi: \mathbb{C} \ell_{r, s} \longrightarrow \operatorname{End}(V)$ be a $*$-morphism). Fix a reference disordered IQPV $\left(J_{\text {ref }} ; \phi\right)$ of (complex) symmetry index $(r, s)$. For any disordered IQPV $(J ; \phi)$ of symmetry index $(r, s)$, define its boundary class by

$$
[(J ; \phi)]_{\partial}:=[(\widehat{J} ; \phi)]-\left[\left(\widehat{J}_{\text {ref }} ; \phi\right)\right] \in K R_{s-r+1}\left(\mathbb{A}_{\partial}\right) \quad\left(\text { resp. } \in K_{s-r+1}\left(\mathrm{~A}_{\partial}\right)\right) .
$$

Here, $[(\widehat{J} ; \phi)]$ and $\left[\left(\widehat{J}_{\text {ref }} ; \phi\right)\right]$ are the classes represented by the corresponding disordered IQPV with boundary of (complex) symmetry index $(r, s)$.

Remark 16. Readers inclined towards physics will wonder how it is possible to assign a class in the $K$-theory of the boundary algebra $\mathbb{A}_{\partial}$ to a disordered IQPV in a given symmetry class, as this would seem to contradict the boundary-anomalous nature of a non-trivial topological phase. Observe, however, that the boundary class is represented by an operator $\widehat{J}$ lying in (the multiplier algebra of) the tensor product $\mathbb{A}_{\partial} \otimes \mathbb{K}\left(\ell^{2}(\mathbb{N})\right)$, rather than in $\mathbb{A}_{\partial}$ itself. That is, the boundary phase is not exactly localised at the boundary, but instead spread out in its vicinity.

For any disordered IQPV with boundary $\left(\widehat{J}^{\prime} ; \phi\right)$ of the same symmetry index, with $\rho\left(\widehat{J}^{\prime}\right)=J$, we have

$$
\rho\left(\widehat{J}^{\prime}-\widehat{J}\right)=0
$$

Thus, $K:=\widehat{J^{\prime}}-\widehat{J} \in \mathbb{A}_{\partial} \otimes \mathbb{K}$. The homotopy $[0,1] \ni t \longmapsto \widehat{J}+t K$ induces a homotopy between $(\widehat{J} ; \phi)$ and $\left(\widehat{J}^{\prime} ; \phi\right)$. Thus $\left(\widehat{J}^{\prime} ; \phi\right)$ and $(\widehat{J} ; \phi)$ define the same class. In this sense, the boundary class for a given bulk IQPV is unique.

Example 1 (Boundary classification for symmetry class $B D \mathrm{I}$ ). For the symmetry class $B D I$, up to stable equivalence, we may choose any symmetry index $(r, s)$ where $r-s \equiv 1$ $(\bmod 8)$, for instance $(r, s)=(1,0)$.

Recalling the proof of Proposition 9, the $K K R$ class corresponding to the $K R$ class represented by the IQPV with boundary $\left(\widehat{J} ; K_{1}\right)$ is

$$
\left[\left(\mathscr{H}_{\mathbb{A}_{\partial}} \otimes C \ell_{0,1}, \psi, \widehat{J} \otimes j_{1}\right)\right] \in K K R\left(C \ell_{0,1}, \mathbb{A}_{\partial} \otimes C \ell_{0,1}\right),
$$

where

$$
\psi: C \ell_{0,1} \longrightarrow \mathrm{M}^{s}\left(\mathbb{A}_{\partial} \otimes C \ell_{0,1}\right)
$$

is determined by $\psi\left(j_{1}\right):=K_{1} \otimes j_{1}$. This class is equivalent to

$$
\left[\left(\mathscr{H}_{\mathbb{A}_{\partial}}, 1_{\mathbb{C}}, \widehat{J} K_{1}\right)\right] \in K K R\left(\mathbb{C}, \mathbb{A}_{\partial}\right),
$$

where $\mathscr{H}_{\mathbb{A}_{\partial}}$ is graded by the operator $K_{1}$. Using an index morphism [49, Theorem 2.2.8], we can identify this $K K R$ class with the class

$$
\left[p^{+}\right]-\left[p^{-}\right] \in K_{0}\left(\mathbb{A}_{\partial}\right)
$$

where $p^{ \pm}$denote the projections onto the submodules $\operatorname{ker}\left(\frac{1}{2}\left(1 \pm K_{1}\right) \widehat{J} K_{1} \frac{1}{2}\left(1 \mp K_{1}\right)\right)$ of the graded real Hilbert $\mathbb{A}_{\partial}$-module $\mathscr{H}_{\mathbb{A}_{\partial}}$. For $K_{1}=\sigma_{z}$, we obtain

$$
\widehat{J} K_{1}=\left(\begin{array}{cc}
0 & \hat{u} \\
\hat{u}^{*} & 0
\end{array}\right), \quad\left[p_{\operatorname{ker}(\hat{u})}\right]-\left[p_{\operatorname{ker}\left(\hat{u}^{*}\right)}\right] \in K_{0}\left(\mathbb{A}_{\partial}\right) .
$$




\section{Bulk-Boundary Correspondence with Disorder}

This section is devoted to the statement and proof of bulk-boundary correspondence for disordered free-fermion topological phases: In the two previous sections, we have attached, to every disordered IQPV with symmetries, real $K$-theory classes in the bulk and at the boundary. Bulk and boundary are connected via the sequence (17). This sequence induces a long exact sequence in real $K$-theory, viz.

$$
\begin{gathered}
\cdots \ldots R_{s-r+2}\left(\mathbb{A}_{\partial}\right) \stackrel{\iota^{*}}{\longrightarrow} K R_{s-r+2}(\widehat{\mathbb{A}}) \stackrel{\varrho^{*}}{\longrightarrow} K R_{s-r+2}(\mathbb{A}) \\
\longrightarrow K R_{s-r+1}\left(\mathbb{A}_{\partial}\right) \stackrel{\iota^{*}}{\longrightarrow} K R_{s-r+1}(\widehat{\mathbb{A}}) \stackrel{\varrho^{*}}{\longrightarrow} K R_{s-r+1}(\mathbb{A}) \cdots \cdots,
\end{gathered}
$$

In particular, we obtain a connecting map

$$
\partial: K R_{s-r+2}(\mathbb{A}) \longrightarrow K R_{s-r+1}\left(\mathbb{A}_{\partial}\right),
$$

which we call the bulk-boundary map. In this section, we will show that $\partial$ maps the bulk class attached to a disordered IQPV with symmetries onto the corresponding boundary class. (The same also holds for the complex symmetry classes.)

Theorem 5 (Bulk-boundary correspondence). Fix a reference disordered IQPV $\left(J_{\text {ref }} ; \phi\right)$ of (complex) symmetry index $(r, s)$. For any disordered IQPV $(J ; \phi)$ of (complex) symmetry index $(r, s)$, we have

$$
\partial[(J ; \phi)]=[(J ; \phi)]_{\partial} .
$$

That is, the bulk-boundary map d maps the bulk class $[(J ; \phi)]$ attached to $(J ; \phi)$ onto the boundary class $[(J ; \phi)]_{\partial}$ attached to $(J ; \phi)$.

5.1. Proof of the main theorem. This subsection is devoted to the proof of Theorem 5. It relies on the use of $K K R$ theory; once again, let us refer to [10,27,49] for accounts thereof. Let us begin by fixing some conventions. Throughout the proof, we will consider the Clifford algebras with their natural grading. All other $C^{*}$-algebras are ungraded. For a real $C^{*}$-algebra $B$, the standard Hilbert $B$-module will be denoted by $\mathscr{H}_{B}$.

Recall Roe's map $\alpha$ from the proof of Theorem 2. Since $\alpha \circ \partial=\partial \circ \alpha$, we can compute the effect of $\partial$ on the bulk class in $D K R$-theory and subsequently apply $\alpha^{-1}$ to obtain a class in $K K R$-theory.

The non-trivial part of the definition of $\alpha$ is given by the connecting map $\partial_{Q}$ in $D K R$ theory. We relate it to the connecting map for the bulk-boundary sequence (17). To that end, recall that $\mathbb{A}_{\partial} \otimes \mathbb{K}\left(\right.$ where $\mathbb{K}=\mathbb{K}\left(\ell^{2}(\mathbb{N})\right)$ ) is an essential ideal in $\widehat{\mathbb{A}}$, as was shown in the proof of Proposition 10. Therefore, the canonical map $\mathbb{A}_{\partial} \otimes \mathbb{K} \longrightarrow \mathbf{M}^{s}\left(\mathbb{A}_{\partial}\right)$ factors as $\Phi \circ \iota$ where $\Phi: \widehat{\mathbb{A}} \longrightarrow \mathrm{M}^{s}\left(\mathbb{A}_{\partial}\right)$ is a unique injective real $*$-morphism.

We obtain a commutative diagram with exact rows:

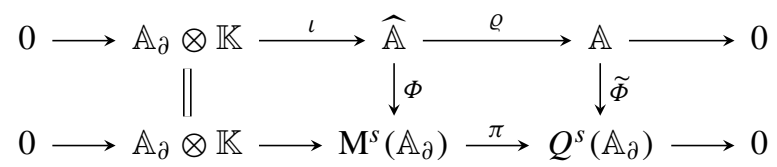


From the naturality of connecting maps (which is a general fact for $\delta$-functors, but can also be derived directly from the explicit form of the connecting map given in [53, Proposition 3.4]), the following diagram commutes:

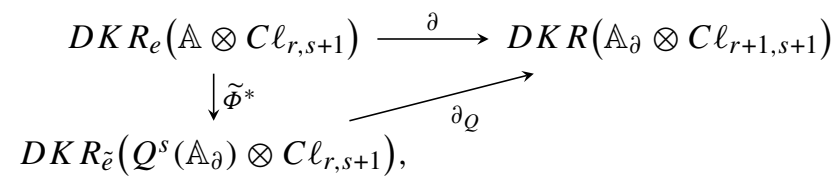

where $\tilde{e}:=\widetilde{\Phi}(e)$. Therefore,

$$
\left(\alpha^{-1} \circ \partial\right)([x]-[e])=\left[\left(\mathscr{H}_{\mathbb{A}_{\partial}} \otimes C \ell_{r, s+1}, 1_{\mathbb{C}}, y\right)\right]-\left[\left(\mathscr{H}_{\mathbb{A}_{\partial}} \otimes C \ell_{r, s+1}, 1_{\mathbb{C}}, f\right)\right]
$$

for $y=\Phi(\hat{x}), f=\Phi(\hat{e}), \varrho(\hat{x})=x$ and $\varrho(\hat{e})=e$. Applying these considerations to the bulk class with reference ORHU $e=J_{\text {ref }} \otimes j_{1} \in \mathscr{F}\left(\mathbb{A} \otimes C \ell_{r, s+1}\right)$, we find by Equation (15) that as an element of $K K R\left(\mathbb{C}, \mathbb{A}_{\partial} \otimes C \ell_{r, s+1}\right)$, the image $\partial[(J ; \phi)]$ of the bulk class under $\partial$ equals

$$
\begin{aligned}
& {\left[\left(\mathscr{H}_{\mathbb{A}_{\partial}} \otimes C \ell_{r, s+1}, 1_{\mathbb{C}},\left(\widehat{J} \otimes j_{1}\right) P+\left(\widehat{J}_{\text {ref }} \otimes j_{1}\right)(1-P)\right)\right]} \\
& \quad-\left[\left(\mathscr{H}_{\mathbb{A}_{\partial}} \otimes C \ell_{r, s+1}, 1_{\mathbb{C}}, \widehat{J}_{\text {ref }} \otimes j_{1}\right)\right]
\end{aligned}
$$

where we write $P:=P^{r, s}$. This in turn equals

$$
\begin{aligned}
& {\left[\left(P\left(\mathscr{H}_{\mathbb{A}_{\partial}} \otimes C \ell_{r, s+1}\right), 1_{\mathbb{C}},\left(\widehat{J} \otimes j_{1}\right) P\right)\right]} \\
& \quad-\left[\left(P\left(\mathscr{H}_{\mathbb{A}_{\partial}} \otimes C \ell_{r, s+1}\right), 1_{\mathbb{C}},\left(\widehat{J}_{\mathrm{ref}} \otimes j_{1}\right) P\right)\right] .
\end{aligned}
$$

We denote the two parts of this difference by $\partial_{\mathrm{I}}$ and $\partial_{\mathbb{I I}}$, respectively. To compare the expression $\partial_{\mathrm{I}}-\partial_{\mathrm{II}}$ to the boundary class, we may treat the parts independently. In what follows, we shall focus on $\partial_{\mathrm{I}}$, as the case of $\partial_{\mathrm{II}}$ differs only in notation.

We set $B:=\mathbb{A}_{\partial} \otimes C \ell_{0,1}$ for brevity. Then the boundary class is given in $K K R\left(C \ell_{s, r}, B\right)$, by the proof of Proposition 9. We will use some standard isomorphisms in $K K$-theory to transfer the class computed above to this $K K R$ group. In preparation thereof, we apply the Clifford algebra isomorphism $C \ell_{r, s+1} \cong C \ell_{0,1} \widehat{\otimes} C \ell_{r, s}$, given by

$$
j_{1} \longmapsto j_{1} \otimes 1, \quad j_{\alpha+1} \otimes 1 \longmapsto 1 \otimes j_{\alpha}, \quad k_{a} \longmapsto-1 \otimes k_{a} .
$$

This isomorphism preserves Clifford orientation in the sense of Kasparov. The image of the projection $P$ now takes the form

$$
P^{\prime}:=\prod_{a=1}^{r} \frac{1}{2}\left(1+(-1)^{s} K_{a} \otimes j_{1} \otimes k_{a}\right) \prod_{\alpha=1}^{s} \frac{1}{2}\left(1+J_{\alpha} \otimes j_{1} \otimes j_{\alpha}\right) .
$$

The first non-trivial isomorphism in $K K$-theory that we apply is the exterior tensor product with the unit class

$$
\tau:=\left[\left(C \ell_{s, r}, \operatorname{id}_{C \ell_{s, r}}, 0\right)\right] \in K K R\left(C \ell_{s, r}, C \ell_{s, r}\right) .
$$

The exterior product $\partial_{\mathrm{I}} \widehat{\otimes} \tau \in K K R\left(C \ell_{s, r}, B \widehat{\otimes} C \ell_{r, s} \widehat{\otimes} C \ell_{s, r}\right)$ equals

$$
\left[\left(\left(P^{\prime} \otimes 1\right)\left(\mathscr{H}_{B} \widehat{\otimes} C \ell_{r, s} \widehat{\otimes} C \ell_{s, r}\right), \mathrm{id}_{C \ell_{s, r}},\left(J \otimes j_{1} \otimes 1 \otimes 1\right)\left(P^{\prime} \otimes 1\right)\right)\right] .
$$


We now apply the orientation-preserving isomorphism $C \ell_{r, s} \widehat{\otimes} C \ell_{s, r} \cong C \ell_{s+r, s+r}$ given by

$k_{a} \otimes 1 \longmapsto(-1)^{s} k_{s+a}, \quad j_{\alpha} \otimes 1 \longmapsto j_{\alpha}, \quad 1 \otimes k_{\alpha} \longmapsto(-1)^{s} k_{\alpha}, \quad 1 \otimes j_{a} \longmapsto j_{s+a}$, for $a=1, \ldots r$ and $\alpha=1, \ldots, s$. This isomorphism converts $P^{\prime} \otimes 1$ into the projection

$$
P^{\prime \prime}=\prod_{a=1}^{r} \frac{1}{2}\left(1+K_{a} \otimes j_{1} \otimes k_{s+a}\right) \prod_{\alpha=1}^{s} \frac{1}{2}\left(1+J_{\alpha} \otimes j_{1} \otimes j_{\alpha}\right),
$$

so it identifies $\partial_{\mathrm{I}} \widehat{\otimes} \tau$ with the class

$$
\left[\left(P^{\prime \prime}\left(\mathscr{H}_{B} \widehat{\otimes} C \ell_{s+r, s+r}\right), \varphi,\left(\widehat{J} \otimes j_{1} \otimes 1\right) P^{\prime}\right)\right] \in K K R\left(C \ell_{s, r}, B \widehat{\otimes} C \ell_{s+r, s+r}\right),
$$

where $\varphi$ is defined by

$$
\varphi\left(k_{\alpha}\right):=(-1)^{s} \otimes 1 \otimes k_{\alpha}, \quad \varphi\left(j_{a}\right)=1 \otimes 1 \otimes j_{s+a} \quad(a=1, \ldots, r, \alpha=1, \ldots, s) .
$$

The next step is to apply a unitary equivalence. We define commuting unitaries

$$
\begin{aligned}
U_{\alpha} & :=\frac{1}{\sqrt{2}}\left(1-J_{\alpha} \otimes j_{1} \otimes k_{\alpha}\right), \quad V_{a}:=\frac{1}{\sqrt{2}}\left(1-K_{a} \otimes j_{1} \otimes j_{s+a}\right) \\
(a & =1, \ldots r, \alpha=1, \ldots, s) .
\end{aligned}
$$

Then we have

$$
\operatorname{Ad}\left(U_{\alpha}\right)\left(1 \otimes 1 \otimes k_{\alpha}\right)=-J_{\alpha} \otimes j_{1} \otimes 1, \quad \operatorname{Ad}\left(V_{a}\right)\left(1 \otimes 1 \otimes j_{s+a}\right)=K_{a} \otimes j_{1} \otimes 1,
$$

whereas $1 \otimes 1 \otimes c$ is fixed for any other generator $c$ of $C \ell_{r+s, r+s}$. Similarly, $\operatorname{Ad}\left(U_{\alpha}\right)$ fixes any $K_{a} \otimes j_{1} \otimes 1$ and $J_{\beta} \otimes j_{1} \otimes 1$ for $\beta \neq \alpha$, and $\operatorname{Ad}\left(V_{a}\right)$ fixes any $J_{\alpha} \otimes j_{1} \otimes 1$ and $K_{b} \otimes j_{1} \otimes 1$ for $b \neq a$. Both actions fix $\widehat{J} \otimes j_{1} \otimes 1$.

Hence, applying the unitary equivalence induced by $V_{1} \cdots V_{r} U_{1} \cdots U_{s}$ identifies $\partial_{\mathrm{I}} \widehat{\otimes} \tau$ with the class

$$
\left[\left(\mathscr{H}_{B} \widehat{\otimes} Q\left(C \ell_{r+s, r+s}\right), \psi \otimes 1, \widehat{J} \otimes j_{1} \otimes Q\right)\right] \in K K R\left(C \ell_{s, r}, B \widehat{\otimes} C \ell_{r+s, r+s}\right)
$$

where $Q=\prod_{i=1}^{r+s} \frac{1}{2}\left(1+k_{i} j_{i}\right)$ and $\psi$ is defined by

$$
\psi\left(k_{\alpha}\right):=(-1)^{s+1} J_{\alpha} \otimes j_{1}, \quad \psi\left(j_{a}\right):=K_{a} \otimes j_{1} \quad(a=1, \ldots, r, \alpha=1, \ldots, s) .
$$

The automorphism of $C \ell_{s, r}$ given by $k_{\alpha} \longmapsto(-1)^{s+1} k_{\alpha}$ and $j_{a} \longmapsto j_{a}$ preserves orientation, as $(-1)^{s(s+1)}=1$, and thus acts trivially on $K K R$. We may therefore remove the signs in the definition of $\psi$ without changing the class.

The final step of the proof will be to apply stability. By [49, Corollary 2.4.9], the stability isomorphism equals the intersection product with the class

$$
\alpha:=[((\mathscr{K} \oplus \Pi \mathscr{K}) \widehat{\otimes} S, 1 \otimes c, T \otimes 1)] \in K K R\left(C \ell_{r+s, r+s}, \mathbb{C}\right) .
$$

Here, $\mathscr{K}$ is a separable Hilbert space, $\Pi \mathscr{K}$ is an odd copy thereof, $(c, S)$ is the unique irreducible real $*$-representation of $C \ell_{r+s, r+s}$ (see Propositions 3 and 5), and

$$
T=\left(\begin{array}{cc}
0 & T_{1}^{*} \\
T_{1} & 0
\end{array}\right), \quad T_{1} T_{1}^{*}=1, \quad T_{1}^{*} T_{1}=1-p,
$$


where $T_{1} \in \mathscr{L}(\mathscr{K})$ and $p$ is a rank one projection. Arguing as in the proof of [49, Theorem 2.4.7], we see that the intersection product with $\alpha$ yields the class

$$
\left[\left(\mathscr{H}_{B} \otimes c(Q)(S), \psi \otimes 1, \widehat{J} \otimes j_{1} \otimes 1\right)\right] \in K K R\left(C \ell_{s, r}, B\right) .
$$

But $c(Q)(S)$ has dimension one and contributes neither to the action nor to the Fredholm operator, so we conclude

$$
\partial_{\mathrm{I}} \widehat{\otimes} \tau \widehat{\otimes}_{C \ell_{r+s, r+s}} \alpha=\left[\left(\mathscr{H}_{B}, \psi, \widehat{J} \otimes j_{1}\right)\right] \in K K R\left(C \ell_{s, r}, B\right) .
$$

The same holds for $\partial_{\mathrm{I}}$ replaced by $\partial_{\mathrm{II}}$ and $\widehat{J}$ replaced by $\widehat{J}_{\text {ref }}$. Because

$$
[(J ; \phi)]_{\partial}=\left[\left(\mathscr{H}_{B}, \psi, \widehat{J} \otimes j_{1}\right)\right]-\left[\left(\mathscr{H}_{B}, \psi, \widehat{J}_{\text {ref }} \otimes j_{1}\right)\right],
$$

this completes the proof of the theorem.

\subsection{Corollaries to the main theorem.}

Corollary 1. Fix $r, s \geqslant 0$ and let the reference IQPV in Theorem 5 be local, i.e. $J_{\text {ref }} \in$ $\mathscr{C}(\Omega) \otimes \operatorname{End}(W)$ (resp. End $(V)$ in the complex case). Then the boundary class simplifies to

$$
[(J ; \phi)]_{\partial}=[(\widehat{J} ; \phi)],
$$

so that bulk-boundary correspondence reads

$$
\partial[(J ; \phi)]=[(\widehat{J} ; \phi)] .
$$

Proof. By assumption, $J_{\text {ref }}$ commutes with $q$, so that $\widehat{J}_{\text {ref }}$ is unitary in $\widehat{\mathbb{A}}$. Hence, the class $\left[\left(\hat{J}_{\text {ref }} ; \phi\right)\right]$ is trivial.

Corollary 2. Fix $r, s \geqslant 0$ and suppose that the reference IQPV in Theorem 5 is local (see the previous corollary). Let $(J ; \phi)$ be a disordered IQPV of (complex) symmetry index $(r, s)$ such that its boundary class is trivial, i.e. $\partial[(J ; \phi)]=0$.

Then $\widehat{J}$ is homotopic to a (real) skew-Hermitian unitary anti-commuting with the $K_{a}, J_{\alpha}$. More precisely, there is a family $\widehat{J_{t}} \in \widehat{\mathbb{A}}, t \in[0,1]$, continuous in the strong-* operator topology, such that $\left(\widehat{J}_{t} ; \phi\right)$ are disordered IQPV with boundary of (complex) symmetry index $(r, s)$, and moreover, $\widehat{J}_{0}=\widehat{J}$ and $\left(\widehat{J}_{1}\right)^{2}=-1$.

Proof. This is immediate from Theorem 5, Corollary 1, and the Theorem of Skandalis [10, Theorem 18.5.3].

Remark 17. Expressed in physics terms, the statement of Corollary 2 is the following: The boundary phase attached to a disordered bulk phase of free fermions is topologically trivial if and only if it exhibits no stable gapless localised boundary states.

Remark 18. By the exactness of the long exact sequence in $K$-theory (19), the image of the map $\partial$ is equal to the kernel of

$$
\iota^{*}: K R_{S-r+1}\left(\mathbb{A}_{\partial}\right) \longrightarrow K R_{S-r+1}(\widehat{\mathbb{A}}) .
$$

By [49, Theorem 1.5.5], this coincides with $\operatorname{ker}\left(\mathrm{id}-\left(\alpha_{d}\right)^{*}\right)$ where the automorphism $\alpha_{d}=\operatorname{Ad}\left(u_{d}\right)$ is defined by considering $\mathbb{A}_{\partial} \subseteq \mathbb{A}$ in a natural fashion. This kernel is trivial if and only if $\alpha_{d}$ is stably homotopic to the identity. While this holds for the clean case without disorder, it may fail in general. Thus, $\partial$ may not be surjective. 
We can compare the boundary phases corresponding to different sides of a boundary. To that end, observe that $1-q$ is the projection corresponding to the left half-space semilattice $-\hat{\Lambda}$. In the following proposition, let $\widehat{J_{+}}:=\widehat{J}$ and $\widehat{J}_{-}:=(1-q) J(1-q)$.

Proposition 11. Let $\left(J_{\mathrm{ref}} ; \phi\right)$ be a reference IQPV of (complex) symmetry index $(r, s)$. For any disordered IQPV $(J ; \phi)$ of (complex) symmetry index $(r, s)$, we have

$$
[(J ; \phi)]_{\partial}^{+}=-[(J ; \phi)]_{\partial}^{-} .
$$

Here, we set

$$
[(J ; \phi)]_{\partial}^{ \pm}:=\left[\left(\widehat{J}_{ \pm} ; \phi\right)\right]-\left[\left(\widehat{J}_{\mathrm{ref}, \pm} ; \phi\right)\right]
$$

Proof. It is sufficient to prove that the sum $\left[\left(\widehat{J}_{+} ; \phi\right)\right]+\left[\left(\widehat{J}_{-} ; \phi\right)\right]$ is trivial for any $J$. The sum is represented by

$$
\left(\widehat{J_{+}} \oplus \widehat{J}_{-}, 1_{2} \otimes \phi\right)
$$

Here, by definition

$$
\widehat{J_{+}} \oplus \widehat{J_{-}}=q J q \oplus(1-q) J(1-q),
$$

where $q$ is the Szegő projection of Definition 17. The right-hand side is the starting point $F_{0}$ of the path

$$
[0,1] \ni t \longmapsto F_{t}:=\left(\begin{array}{cc}
q J q & t q J(1-q) \\
t(1-q) J q(1-q) J(1-q)
\end{array}\right)
$$

The operators $F_{t}$ are skew-Hermitian and

$$
F_{t}^{2}=\left(\begin{array}{cc}
q\left(-1+\left(t^{2}-1\right) J(1-q) J\right) q & 0 \\
0 & (1-q)\left(-1+\left(t^{2}-1\right) J q J\right)
\end{array}\right) .
$$

The operator $q J(1-q) J q$ lies in the kernel of the canonical projection $\varrho=\varrho_{+}$of the right half-space algebra. Indeed, the expression $q O_{1}(1-q) O_{2} q$ vanishes if $O_{1}$ and $O_{2}$ are among the generators $f \in \mathscr{C}(\Omega) \otimes \operatorname{End}(W)$ and $u_{1}, \ldots, u_{d-1}$, together with their adjoints, as all of these commute with $q$. Furthermore, we compute

$$
(1-q) u_{d} q=0, \quad(1-q) u_{d}^{*} q=u_{d}^{*} e .
$$

Because $J$ is the limit of non-commutative polynomials in the generators $f$ and $u_{1}, \ldots, u_{d}$, together with their adjoints, it follows that $q J(1-q) J q$ lies in the closed two-sided ideal generated by $e$. This is the kernel of $\varrho_{+}$by Theorem 4 . A similar statement holds for the lower right corner of the matrix representing $F_{t}^{2}$. Hence, $\left(F_{t}\right)$ is an operator homotopy, so since $F_{1}^{2}=-1$, it follows that the class represented by $F_{0}$ is trivial. 
5.3. Invariance under disorder. So far, the precise nature of the space $\Omega$ of disorder configurations has not been important. Bulk-boundary correspondence holds for any choice of $\Omega$. However, the $K$-theory of the bulk and boundary algebras will in general depend on $\Omega$. We now address a specific type of disorder where this dependence vanishes.

In practice, disorder is introduced chiefly through impurities. In this case, the electrons experience random perturbations to the on-site atomic potential. This leads to the model

$$
\Omega=\Omega_{0}^{\Lambda}=\prod_{x \in \Lambda} \Omega_{0}
$$

for some compact Hausdorff space $\Omega_{0}$, where the action of $\Lambda$ is by translation in the parameter set $\Lambda$. This dynamical system, known in mathematics as a Bernoulli shift, was suggested by Bellissard as a model for the disorder space (see [43] for an exposition).

If $\Omega_{0}$ is the space of possible perturbations of the on-site potential, then, in keeping with the literature, we assume that $\Omega_{0}$ is a compact convex subset of $\mathbb{R}^{d}$. (The physical justification for this assumption is discussed at length in [35].) Mathematically, one may just as well make the following more general hypothesis.

Proposition 12. Suppose that $\Omega$ is a Bernoulli shift with contractible $\Omega_{0}$. Let $\mathbb{A}^{0}, \widehat{\mathbb{A}}^{0}$, and $\mathbb{A}_{\partial}^{0}$ denote the algebras $\mathbb{A}, \widehat{\mathbb{A}}$, and $\mathbb{A}_{\partial}$, respectively, for the case of no disorder (i.e., where $\Omega$ is a one-point space). There is a commutative diagram

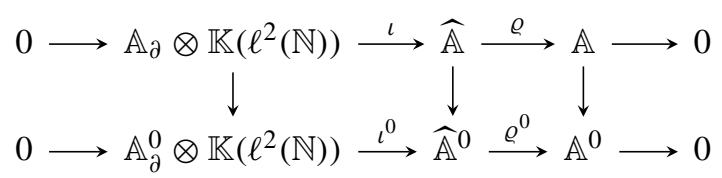

where the rows are the short exact sequence (17) for $\mathbb{A}$ and $\mathbb{A}^{0}$, respectively, and the vertical maps are homotopy equivalences of real $C^{*}$-algebras.

In particular, the bulk-boundary map $\partial$ is surjective and

$$
\begin{aligned}
K R_{s-r+2}(\mathbb{A}) & =K R_{s-r+2}\left(\mathbb{A}^{0}\right)=\bigoplus_{i=0}^{d}\left(\begin{array}{l}
d \\
i
\end{array}\right) K R_{s-r+2-i}(\mathbb{C}), \\
K_{S}(\mathrm{~A}) & =K_{s}\left(\mathrm{~A}^{0}\right)=\mathbb{Z}^{2^{d-1}}
\end{aligned}
$$

Remark 19. Equation (22) identifies $K R_{s-r+2}(\mathbb{A})$ with the real $K$-theory of the $d$ dimensional (Brillouin) torus. The summands correspond to the well-known $K O$ groups of the point, see for example [4, Table 2]. The component of the bulk class of a disordered IQPV $(J ; \phi)$ of symmetry index $(r, s)$ corresponding to the summand for $i=d$ is what is often referred to in the literature as the strong invariant. The other components are summarily called weak invariants. As Theorem 5 and Proposition 12 show, at least for the type of disorder considered here, this distinction is not mathematically compelling.

In [43, Proposition 4.2.4], the $K$-theory of the bulk algebra A is computed under the assumption that $\Omega$ is a Bernoulli shift with $\Omega_{0}$ a convex compact set. The proof is based on the Pimsner-Voiculescu exact six-term sequence and relies on the statement [43, Proposition 4.2.1] that the action $\alpha_{d}$ of the translation in the direction transverse to the boundary on $\mathscr{C}(\Omega)$ is homotopic to the identity. It is however not true in general that the homotopy $\xi_{t}$ given in the proof is actually an isotopy on $\Omega$. For instance, let $d=1$, $\Omega_{0}=[-1,1]$, and $\omega_{n}:=(-1)^{n}$. Then $\xi_{1 / 2}(0)=0$, but also $\xi_{1 / 2}(\omega)=0$, so that $\xi_{1 / 2}$ is not injective. Hence, the proof as given in [43] appears to be incomplete. 
Proof of Proposition 12. By hypothesis, there is a point $\omega_{0} \in \Omega$ and a homotopy $h=$ $\left(h_{t}\right)$ of maps $\Omega_{0} \longrightarrow \Omega_{0}$ where $h_{0}=\omega_{0}$ is the constant map and $h_{1}=\mathrm{id}_{\Omega_{0}}$.

Consider the inclusion $\eta: \operatorname{End}(W) \longrightarrow \mathscr{C}(\Omega) \otimes \operatorname{End}(W)$ and the evaluation

$$
\varepsilon: \mathscr{C}(\Omega) \otimes \operatorname{End}(W) \longrightarrow \operatorname{End}(W): f \longmapsto f\left(x \longmapsto \omega_{0}\right)
$$

at the constant sequence $x \longmapsto \omega_{0}$. We have $\varepsilon \circ \eta=\mathrm{id}$. Moreover, for any $t \in[0,1]$,

$$
\phi_{t}(f)(\omega):=f\left(h_{t}(\omega)\right) \quad(f \in \mathscr{C}(\Omega) \otimes \operatorname{End}(W), \omega \in \Omega)
$$

defines a homotopy $\left(\phi_{t}\right)$ of real $*$-endomorphisms of $\mathscr{C}(\Omega) \otimes \operatorname{End}(W)$ such that

$$
\phi_{0}=\eta \circ \varepsilon, \quad \phi_{1}=\operatorname{id}_{\mathscr{C}}(\Omega) \otimes \operatorname{End}(W) .
$$

By definition, the morphisms $\varepsilon, \eta$, and $\phi_{t}$ are $\Lambda$-equivariant, and by restriction, also equivariant with respect to $\Lambda_{\partial}$ and $\hat{\Lambda}$. By Theorem 1 , Proposition 8 , and Theorem 4 , the existence of the diagram (21) follows.

The homotopy invariance of $K$-theory gives $K R_{\bullet}(\mathbb{A})=K R_{\bullet}\left(\mathbb{A}^{0}\right)$. In view of [49, Theorem 1.5.5], $\partial$ is surjective. Since by Theorem 1 ,

$$
\mathbb{A}^{0}=(\mathbb{C} \rtimes \Lambda) \otimes \operatorname{End}(W)=C^{*}(\Lambda) \otimes \operatorname{End}(W),
$$

where $C^{*}(\Lambda)$ is the group $C^{*}$-algebra of $\Lambda$, we find that

$$
K R_{\bullet}(\mathbb{A})=K R_{\bullet}\left(C^{*}(\Lambda)\right)=K R_{\bullet}\left(\bigotimes^{d} \mathscr{C}\left(\mathbb{S}^{1}\right)\right) .
$$

From this, Equation (22) follows by [49, Theorem 1.5.4]. Equation (23) is a special case.

\section{Discussion}

We have derived a framework for the bulk and boundary classification of disordered free-fermion topological phases and proved that these are naturally related. In this final section, we complement our previous results by a discussion, and by drawing some physical conclusions.

6.1. Anomalous nature of the boundary phase. The physicists among our readers may wonder how to interpret our main result from the heuristics of anomaly inflow. Indeed, built on a tight-binding model, the boundary algebra $\mathbb{A}_{\partial}$ is UV-complete and as such does not allow for the anomaly characteristic of the spatial boundary of a system in a non-trivial topological phase. Yet, the bulk-boundary correspondence established in Theorem 5 does assign to the anomalous system boundary a class in the $K R$-theory of the algebra $\mathbb{A}_{\partial}$.

To resolve the apparent conflict, one may observe that whereas the bulk $K$-theory class of a free-fermion ground state in symmetry class $s$ resides in degree $s+2$, the degree of the boundary class is shifted by one to $s+1$. The index shift may be traded for a suspension, viz.

$$
K R_{s+1}\left(\mathbb{A}_{\partial}\right)=K R_{S+2}\left(S \mathbb{A}_{\partial}\right),
$$

where the suspension $S$ is effected by the tensor product with the algebra $\mathscr{C}_{0}(i \mathbb{R})$. So, morally, the boundary phase is not represented by a vector bundle (with the corresponding Clifford symmetries) over the boundary; rather, it is represented by a vector bundle over the suspension of the boundary. 
6.2. The role of the trivial phase. Our construction of bulk classes depends on the choice of a trivial phase. Indeed, we have constructed bulk classes as deviations from an arbitrarily chosen reference IQPV. This circumstance is imposed by the group structure of $K$-theory, which measures differences of certain homotopy classes, rather than the classes themselves.

From a physical point of view, free-fermion topological phases do not directly have a group structure, whereas differences of such phases do. Therefore, it is advantageous for the physical interpretation of the bulk classes as free-fermion topological phases that the choice of reference IQPV is not fixed by the mathematical model, and instead allows for adjustments according to the physical system of interest.

The situation is different for the boundary classes. Any reference disordered IQPV $J_{\text {ref }}$ that has a unitary lift $\widehat{J}_{\text {ref }}$ (in particular, any local reference IQPV in the sense of Corollaries 1 and 2) will itself already represent a degenerate Kasparov class, trivial in the associated $K$-group. This corresponds to the understanding in physics that a boundary free-fermion topological phase is trivial if there are no stable localised boundary states that close the gap.

To state bulk-boundary correspondence cleanly, our definition of boundary classes avoids the requirement that the reference IQPV be trivial in this sense. It instead measures the deviation from an arbitrary reference boundary class. The bulk-boundary map is sensitive to the choice of reference phase and preserves this dependence.

6.3. The role of the internal space $W$. Throughout this article, we have relied on a tightbinding approximation in which the relevant degrees of freedom close to the chemical potential split into a spatial contribution, corresponding to the translational lattice of atomic sites $\Lambda$, and a finite-dimensional internal space $V$ resp. $W=V \oplus V^{*}$ independent of the spatial part. It is important that the choice of this splitting is performed in such a way that the only relevant spatial symmetries (in mean) of the Hamiltonians of interest are the lattice translations.

This is shown by the following Gedankenexperiment due to Fu-Kane-Mele [20]. Assume that $W$ is doubled to $W_{2}:=W \oplus W$ while $\Lambda$ is reduced to $\Lambda_{2}$ by merging atomic positions adjacent in one selected spatial direction. Let $\mathbb{A}_{2}$ be the bulk algebra defined in terms of $W_{2}$ and $\Lambda_{2}$, and suppose we are in the setting of Proposition 12. Although the $K$-groups of $\mathbb{A}$ and $\mathbb{A}_{2}$ are isomorphic by the proposition, the natural "doubling" map $\phi_{2}: \mathbb{A} \longrightarrow \mathbb{A}_{2}$ is not an isomorphism, not even on $K$-theory. Indeed, $\mathrm{Fu}-\mathrm{Kane}-\mathrm{Mele}$ show for clean systems that, although the strong invariant is preserved, some weak invariants are annihilated by doubling; by the proposition, this also holds in the presence of disorder. Fu-Kane-Mele view the annihilation of weak invariants as an indication that they are not stable under disorder. However, as we have seen, all invariants are preserved by the kind of disorder we consider. This is corroborated by the work of Ringel-Kraus-Stern [45].

The problem lies in the fact that $\phi_{2}$ maps an IQPV $J$ in $\mathbb{A}$ to one in the algebra $\mathbb{A}_{2}$ which forgets the invariance (in mean) of $J$ under the translations removed by the doubling process. By incorporating these lost translations into the definition of the algebra as symmetries of $\mathscr{W}_{2}=\ell^{2}\left(\Lambda_{2}\right) \otimes W_{2} \cong \mathscr{W}$, we obtain a new algebra, isomorphic to $\mathbb{A}$ under the doubling map, thus removing the perceived inconsistencies.

6.4. Numerical topological invariants. In this paper, we have defined bulk and boundary classes for disordered IQPV in the symmetry classes of the Tenfold Way. For applica- 
tions, it is desirable to have numerical indices available which make this topological classification amenable to computation. The derivation and in-depth study of all such indices for our topological classes is beyond the scope of this paper. Instead, we shall give an overview of the literature and then discuss the prominent special case of the strong topological phase in some detail.

There are two major approaches to the construction of numerical topological indices. One uses the pairing with cyclic cohomology introduced by Connes, see [18, Chapter 3.3]. Attached to an algebra $A$, there are cyclic cohomology groups $H C^{n}(A)$, dual to $K$-theory in the sense that there is a canonical pairing

$$
K_{n}(A) \otimes H C^{n}(A) \longrightarrow \mathbb{C} \quad(n=0,1) .
$$

This pairing has been used to write the quantised Hall conductance in the IQHE, expressed in units of $\frac{e^{2}}{h}$, as the result of pairing the $K$-theory class defined by the QHE Hamiltonian with a canonical cyclic co-cycle $[8,43]$.

Although a pairing with cyclic cohomology can also be defined for the $K R$-groups of real $K$-theory appearing in the context of the present work, the result is unsatisfactory. Indeed, the $K R$-group of the bulk algebra may have torsion, that is, it may contain non-zero elements of finite order. For instance, the $K R$-theory of the torus has $\mathbb{Z} / 2 \mathbb{Z}$ summands in certain degrees. Any $\mathbb{C}$ - or $\mathbb{Z}$-valued pairing will therefore be degenerate. Kellendonk addresses this problem in recent work [29].

A different approach to the construction of numerical topological invariants is to replace cyclic cohomology by the $K$-homology groups $K R^{n}(\mathbb{A})=K K R\left(\mathbb{A} \otimes C \ell_{0, n}, \mathbb{C}\right)$ of the bulk algebra $\mathbb{A}$ and to use the Kasparov product

$$
K K R\left(C \ell_{s, r}, \mathbb{A} \otimes C \ell_{0,1}\right) \otimes K K R\left(\mathbb{A} \otimes C \ell_{0, n}, \mathbb{C}\right) \longrightarrow K R_{s+1-r-n}(\mathbb{C}) .
$$

Recall here that $K R_{s+1-r}(\mathbb{A})=K K R\left(C \ell_{s, r}, \mathbb{A} \otimes C \ell_{0,1}\right)$ is the group harbouring the bulk classes of free-fermion topological phases that we have constructed. In principle, this approach avoids the problems with $\mathbb{C}$ - or $\mathbb{Z}$-valued pairings, as the right-hand side may be any summand appearing in the $K R$-theory of the torus.

The difficulty lies in finding suitable classes in $K$-homology to insert into the pairing. For $n=d$, where $d$ is the dimension of lattice $\Lambda$ of translations, such a $K$-homology class is constructed in [12], leading to an index formula for the strong topological invariant. A complete picture is as yet unavailable for the case of $n<d$ corresponding to the weak invariants, although some contributions to this end have been made in [14].

Let us examine the strong invariant more closely. As we have seen above, in the clean limit, it is defined as the top-dimensional $K$-theory invariant of the $d$-dimensional torus. As long as the disorder space $\Omega$ meets the assumptions of Proposition 12, this definition still makes sense. In general, the definition depends on the choice of a point $\omega \in \Omega$ fixed by the action of the translational lattice $\Lambda$. (For the Bernoulli shifts of Proposition 12, the fixed points are the constant sequences.) Evaluation at $\omega$ defines a map

$$
\lambda_{d-1}^{\omega}: K R_{S-r+1}\left(\mathbb{A}_{\partial}\right) \longrightarrow K R_{s-r+2-d}(\mathbb{C})
$$

generalising the strong invariant [13].

As the notation suggests, $\lambda_{d-1}^{\omega}$ might depend on $\omega$. In the situation of Proposition 12, it is independent thereof. More generally, if the action of $\Lambda$ on $\Omega$ is ergodic for an invariant probability $\mathbb{P}$ on $\Omega$, then it is $\mathbb{P}$-a.s. constant as a function of $\omega$. (This is really a generalisation of the former statement, as Bernoulli shifts admit ergodic measures.)

So far, we have assumed that the boundary is perpendicular the $d$ th coordinate direction. For a fixed choice of disordered IQPV of a given symmetry index, one may observe 
topological features at different boundaries. Although they may in general depend on the choice of the boundary, we have for the strong topological invariant:

$$
(-1)^{i} \lambda_{d-1}^{\omega} \circ \partial_{i}=(-1)^{j} \lambda_{d-1}^{\omega} \circ \partial_{j} \quad(i, j=1, \ldots, d),
$$

as follows from [13, Corollary 3.6]. Here, $\partial_{i}$ is the bulk-boundary map for a boundary plane perpendicular to the $i$ th coordinate axis. Thus, pull-backs to the bulk of the strong topological invariants for different boundaries differ only by the sign corresponding to the change of orientation. From our explicit form of the bulk-boundary correspondence, we conclude that all boundaries exhibit gapless boundary states, as long as the strong topological bulk phase is non-trivial.

Acknowledgements. The authors wish to thank Matthias Lesch for his remarks on Lemma 5 in a previous version of the manuscript, which have helped us improve a statement which otherwise could easily have led to misconceptions. Our thanks also extend to an anonymous referee, whose comments have aided us in enhancing the article. Research supported by Deutsche Forschungsgemeinschaft (DFG), Projektnummer 316511131, TRR 183, project A03 (all authors), Projektnummer 282448306, AL 698/3-1 (AA), and Projektnummer 49599460, GSC 260 (CM), as well as QM²-Quantum Matter and Materials as part of the Institutional Strategy of the University of Cologne in the Excellence Initiative (all authors). We (AA, CM) thank the Department of Mathematics of the University of California at Berkeley and the Institute for Theoretical Physics at the University of Cologne for their hospitality during the preparation of this article. CM acknowledges the participation in the School on " $K K$-theory, Gauge Theory, and Topological Phases" (February 27-March 10, 2017 ) at the Lorentz Center, Leiden, and the Thematic Programme on "Bivariant $K$-theory in Geometry and Physics" (November 6-30, 2018) at the Erwin Schrödinger Institute for Mathematical Physics, Vienna.

Open Access This article is distributed under the terms of the Creative Commons Attribution 4.0 International License (http://creativecommons.org/licenses/by/4.0/), which permits unrestricted use, distribution, and reproduction in any medium, provided you give appropriate credit to the original author(s) and the source, provide a link to the Creative Commons license, and indicate if changes were made.

Publisher's Note Springer Nature remains neutral with regard to jurisdictional claims in published maps and institutional affiliations.

\section{References}

1. Altland, A., Zirnbauer, M.R.: Nonstandard symmetry classes in mesoscopic normal-superconducting hybrid structures. Phys. Rev. B 55(2), 1142-1161 (1997)

2. Arens, R.: Representation of *-algebras. Duke Math. J. 14, 269282 (1947)

3. Arens, R.F., Kaplansky, I.: Topological representation of algebras. Trans. Am. Math. Soc. 63, 457-481 (1948)

4. Atiyah, M.F., Bott, R., Shapiro, A.: Clifford modules. Topology 3(suppl. 1), 3-38 (1964). https://doi.org/ 10.1016/0040-9383(64)90003-5

5. Avron, J.E., Seiler, R., Simon, B.: Homotopy and quantization in condensed matter physics. Phys. Rev. Lett. 51(1), 51-53 (1983). https://doi.org/10.1103/PhysRevLett.51.51

6. Bellissard, J.: Gap labelling theorems for Schrödinger operators. In: Waldschmidt, M., Moussa, P., Luck, J.M., Itzykson, C. (eds.) From Number Theory to Physics (Les Houches, 1989), pp. 538-630. Springer, Berlin, Heidelberg (1992). https://doi.org/10.1007/978-3-662-02838-4_12

7. Bellissard, J.: K-theory of $C^{*}$-algebras in solid state physics. In: Dorlas, T.C., Hugenholtz, N.M., Winnink, M. (eds.) Statistical Mechanics and Field Theory: Mathematical Aspects (Groningen, 1985). Lecture Notes Physics, vol. 257, pp. 99-156. Springer, Berlin, Heidelberg (1986). https://doi.org/10.1007/3-54016777-3_74

8. Bellissard, J., van Elst, A., Schulz-Baldes, H.: The noncommutative geometry of the quantum Hall effect. J. Math. Phys. 35(10), 5373-5451 (1994). https://doi.org/10.1063/1.530758

9. Bellissard, J., Nakamura, S.: Low energy bands do not contribute to quantum Hall effect. Commun. Math. Phys. 131(2), 283-305 (1990)

10. Blackadar, B.: K-Theory for Operator Algebras. Mathematical Sciences Research Institute Publications. Cambridge University Press, Cambridge (1998) 
11. Blackadar, B.: Shape theory for $C^{*}$-algebras. Math. Scand. 56(2), 249-275 (1985). https://doi.org/10. 7146/math.scand.a-12100

12. Bourne, C., Carey, A.L., Rennie, A.: A non-commutative framework for topological insulators. Rev. Math. Phys. (2016). https://doi.org/10.1142/S0129055X16500045

13. Bourne, C., Kellendonk, J., Rennie, A.: The K-theoretic bulk-edge correspondence for topological insulators. Ann. Inst. Henri Poincare (A) 18, 1833-1866 (2017). https://doi.org/10.1007/s00023-016-05412

14. Bourne, C., Schulz-Baldes, H.: Application of semifinite index theory to weak topological phases. In: Wood, D., de Gier, J., Praeger, C., Tao, T. (eds.) 2016 MATRIX Annals. MATRIX Book Series, vol. 1, pp. 203-227. Springer, Cham (2018). https://doi.org/10.1007/978-3-319-72299-3_10

15. Busby, R.C.: Double centralizers and extensions of $C^{*}$-algebras. Trans. Am. Math. Soc. 132, 79-99 (1968). https://doi.org/10.2307/1994883

16. Busby, R.C., Smith, H.A.: Representations of twisted group algebras. Trans. Am. Math. Soc. 149, 503-537 (1970). https://doi.org/10.2307/1995410

17. Chiu, C.-K., et al.: Classification of topological quantum matter with symmetries. Rev. Mod. Phys. 88(3), 035005 (2016). https://doi.org/10.1103/RevModPhys.88.035005

18. Connes, A.: Noncommutative Geometry. Elsevier Science, Amsterdam (1995). ISBN 9780080571751

19. Freed, D.S., Moore, G.W.: Twisted equivariant matter. Ann. Henri Poincare 14(8), 1927-2023 (2013). https://doi.org/10.1007/s00023-013-0236-x

20. Fu, L., Kane, C.L., Mele, E.J.: Topological insulators in three dimensions. Phys. Rev. Lett. 98, 106803 (2007). https://doi.org/10.1103/PhysRevLett.98.106803

21. Graf, G.M., Porta, M.: Bulk-edge correspondence for two-dimensional topological insulators. Commun. Math. Phys. 324(3), 851-895 (2013). https://doi.org/10.1007/s00220-013-1819-6

22. Haldane, F.D.M.: Model for a quantum Hall effect without Landau levels condensed-matter realization of the 'parity anomaly. Phys. Rev. Lett. 61(18), 2015-2018 (1988)

23. Hannabuss, K.C., Mathai, V., Thiang, G.C.: T-duality simplifies bulk-boundary correspondence: the noncommutative case. Lett. Math. Phys. 108(5), 1163-1201 (2018). https://doi.org/10.1007/s11005-0171028-x

24. Heinzner, P., Huckleberry, A., Zirnbauer, M.R.: Symmetry classes of disordered fermions. Commun. Math. Phys. 257(3), 725-771 (2005). https://doi.org/10.1007/s00220-005-1330-9

25. Ingelstam, L.: Real Banach algebras. Ark. Mat. 5, 239-270 (1964)

26. Kane, C.L., Mele, E.J.: $\mathbb{Z} / 2 \mathbb{Z}$ topological order and the quantum spin Hall effect. Phys. Rev. Lett. 95(14), 146802 (2005). https://doi.org/10.1103/PhysRevLett.95.146802

27. Kasparov, G.G.: The operator $K$-functor and extensions of $C^{*}$-algebras. Izv. Akad. Nauk SSSR Ser. Mat. 16(3), 513-572 (1981)

28. Kasparov, G.G.: Topological invariants of elliptic operators. I. $K$-homology. Izv. Akad. Nauk SSSR Ser. Mat. 9(4), 751-792 (1975)

29. Kellendonk, J.: Cyclic cohomology for graded $C^{*, r}$-algebras and its pairings with van Daele K-theory. Commun. Math. Phys. 368(2), 467-518 (2019). https://doi.org/10.1007/s00220-019-03452-1

30. Kellendonk, J.: On the $\mathrm{C}^{*}$-algebraic approach to topological phases for insulators. Ann. Henri Poincare 18(7), 2251-2300 (2017). https://doi.org/10.1007/s00023-017-0583-0

31. Kellendonk, J., Richter, T., Schulz-Baldes, H.: Edge current channels and Chern numbers in the integer quantum Hall effect. Rev. Math. Phys. 14(1), 87-119 (2002). https://doi.org/10.1142/ S0129055X02001107

32. Kennedy, R., Zirnbauer, M.R.: Bott periodicity for $\mathbb{Z}_{2}$ symmetric ground states of gapped free-fermion systems. Commun. Math. Phys. 342, 909-963 (2016). https://doi.org/10.1007/s00220-015-2512-8

33. Kitaev, A.: Periodic table for topological insulators and superconductors. AIP Conf. Proc. 1134(1), 22-30 (2009)

34. Kubota, Y.: Controlled topological phases and bulk-edge correspondence. Commun. Math. Phys. 349(2), 493-525 (2017). https://doi.org/10.1007/s00220-016-2699-3

35. Kuhne, T.D., Prodan, E.: Disordered crystals from first principles I: quantifying the configuration space. Ann. Phys. 391, 120-149 (2018). https://doi.org/10.1016/j.aop.2018.01.016

36. Lawson Jr., H.B., Michelsohn, M.-L.: Spin Geometry. Vol. 38. Princeton Mathematical Series. Princeton University Press, Princeton (1989)

37. Loring, T.A.: $K$-theory and pseudospectra for topological insulators. Ann. Phys. 356, 383-416 (2015). https://doi.org/10.1016/j.aop.2015.02.031

38. Luke, G.M., et al.: Time-reversal symmetry breaking superconductivity in $\mathrm{Sr}_{2} \mathrm{RuO}_{4}$. Nature 394(6693), 558-561 (1998). https://doi.org/10.1038/29038

39. Mathai, V., Thiang, G.C.: $T$-duality simplifies bulk-boundary correspondence: some higher dimensional cases. Ann. Henri Poincare 17(12), 3399-3424 (2016). https://doi.org/10.1007/s00023-016-0505-6

40. Mathai, V., Thiang, G.C.: T-duality simplifies bulk-boundary correspondence. Commun. Math. Phys. 345(2), 675-701 (2016). https://doi.org/10.1007/s00220-016-2619-6 
41. Murphy, G.J.: Ordered groups and crossed products of $C^{*}$-algebras. Pac. J. Math. 148(2), 319-349 (1991)

42. Pedersen, G.K.: $C *$-Algebras and Their Automorphism Groups, Vol. 14. London Mathematics Society of Monographs. Academic Press Inc., London (1979)

43. Prodan, E., Schulz-Baldes, H.: Bulk and Boundary Invariants for Complex Topological Insulators. Mathematical Physics Studies. Springer, Cham (2016). https://doi.org/10.1007/978-3-319-29351-6

44. Raeburn, I., Williams, D.P.: Morita Equivalence and Continuous-Trace $C^{*}$-Algebras. Vol. 60. Mathematical Surveys and Monographs. American Mathematical Society, Providence (1998). https://doi.org/10. 1090/surv/060

45. Ringel, Z., Kraus, Y.E., Stern, A.: Strong side of weak topological insulators. Phys. Rev. B 86, 045102 (2012). https://doi.org/10.1103/PhysRevB.86.045102

46. Roe, J.: Lectures on Coarse Geometry. Vol. 10. University Lecture Series. American Mathematical Society, Provoidence (2003). https://doi.org/10.1090/ulect/031

47. Roe, J.: Paschke duality for real and graded $C *$-algebras. Q. J. Math. Oxf. Ser. 55(3), 325-331 (2004). https://doi.org/10.1093/qmath/hah001

48. Schnyder, A.P., et al.: Classification of topological insulators and superconductors in three spatial dimensions. Phys. Rev. B 78(19), 195125 (2008)

49. Schroder, H.: $K$-Theory for Real $C *$-Algebras and Applications. Chapman \& Hall/CRC Research Notes in Mathematics Series. Taylor \& Francis, Abingdon (1993)

50. Schulz-Baldes, H., Kellendonk, J., Richter, T.: Simultaneous quantization of edge and bulk Hall conductivity. J. Phys. A 33(2), L27-L32 (2000). https://doi.org/10.1088/0305-4470/33/2/102

51. Thouless, D.J., et al.: Quantized Hall conductance in a two-dimensional periodic potential. Phys. Rev. Lett. 49(6), 405-408 (1982). https://doi.org/10.1103/PhysRevLett.49.405

52. Van Daele, A.: K-theory for graded Banach algebras. I. Q. J. Math. Oxf. Ser. (2) 39(154), 185-199 (1988). https://doi.org/10.1093/qmath/39.2.185

53. Van Daele, A.: $K$-theory for graded Banach algebras. II. Pac. J. Math. 134(2), 377-392 (1988). DOI: euclid.pjm/1102689267

Communicated by Y. Kawahigashi 